\title{
Weak turbulence theory for collisional plasmas
}

\author{
P. H. Yoon, ${ }^{1,2, *}$ L. F. Ziebell, ${ }^{3}$ E. P. Kontar, ${ }^{4}$ and R. Schlickeiser ${ }^{5}$ \\ ${ }^{1}$ IPST, University of Maryland, College Park, Maryland 20742, USA \\ ${ }^{2}$ School of Space Research, Kyung Hee University, Yongin, Gyeonggi 446-701, Republic of Korea \\ ${ }^{3}$ Instituto de Física, Universidade Federal do Rio Grande do Sul, 91501-970 Porto Alegre, RS, Brazil \\ ${ }^{4}$ School of Physics and Astronomy, University of Glasgow, Glasgow G12 8QQ, UK \\ ${ }^{5}$ Institut für Theoretische Physik, Lehrstuhl IV: Weltraum- und Astrophysik, Ruhr-Universität Bochum, D-44780 Bochum, Germany
}

(Received 15 October 2015; revised manuscript received 25 January 2016; published 8 March 2016)

\begin{abstract}
Plasma is an ionized gas in which the collective behavior dominates over the individual particle interactions. For this reason, plasma is often treated as collisionless or collision-free. However, the discrete nature of the particles can be important, and often, the description of plasmas is incomplete without properly taking the discrete particle effects into account. The weak turbulence theory is a perturbative nonlinear theory, whose essential formalism was developed in the late 1950s and 1960s and continued on through the early 1980s. However, the standard material found in the literature does not treat the discrete particle effects and the associated fluctuations emitted spontaneously by thermal particles completely. Plasma particles emit electromagnetic fluctuations in all frequencies and wave vectors, but in the standard literature, the fluctuations are approximately treated by considering only those frequency-wave number regimes corresponding to the eigenmodes (or normal modes) satisfying the dispersion relations, while ignoring contributions from noneigenmodes. The present paper shows that the noneigenmode fluctuations modify the particle kinetic equation so that the generalized equation includes the Balescu-Lénard-Landau collision integral and also modify the wave kinetic equation to include not only the collisional damping term but also a term that depicts the bremsstrahlung emission of plasma normal modes.
\end{abstract}

DOI: 10.1103/PhysRevE.93.033203

\section{INTRODUCTION}

In the context of kinetic theory, plasmas can be considered collisionless if one is interested in the study of phenomena that evolve on time scales much shorter than time scales of binary collisions. For such cases, the Vlasov-Maxwell system of equations, which is a complex set of coupled equations, may be employed. The Vlasov equation is a nonlinear partial differential equation, which is customarily solved under different degrees of approximation. Under the linear approximation, which is the simplest, it is possible to obtain the dispersion relations, which allow the identification of different modes of oscillations (i.e., eigenmodes or normal modes) that may be excited in a plasma, and how they propagate. Well-known examples may be the high-frequency oscillations known as the Langmuir wave $(L)$ and the low-frequency oscillations called the ion-acoustic or ion-sound wave $(S)$. These are both longitudinal electrostatic oscillations. Another well-known example may be the Alfvén wave, which is a low-frequency transverse wave that exists in a magnetized plasma. Of course, plasmas excite a host of other complicated normal modes, especially when it is immersed in an ambient magnetic field.

The dispersion relations obtained by employing linear theory provide information on the characteristics of these modes of oscillation and describe conditions under which such waves exponentially damp or grow, given a state of the plasma. However, linear theory cannot provide the necessary information about the time evolution of the system or how unstable oscillations saturate. For these one needs to incorporate nonlinear effects. The lowest order approach to incorporating nonlinear effects is the quasilinear theory,

*yoonp@umd.edu which utilizes adiabatic dispersion relations that are formally identical to those described by the linear theory, but which depend on velocity distribution functions that evolve in time, on a time scale slower than that which characterizes the periods of plasma oscillations. The time evolution of the particle distribution function is governed by a diffusion equation in velocity space, with a diffusion coefficient that depends on the spectral distribution of wave energy.

The subsequent step in this chain of approximations is the weak turbulence theory, which incorporates nonlinear effects of lowest order, that is, the quadratic nonlinearity. It is represented by a set of coupled equations that describe the time evolution of particle distribution functions and the time evolution of spectral intensities of the wave modes. The weak turbulence theory was largely developed between the late 1950s and the 1970s [1-10] and has been used since then in many studies of plasma phenomena, which are ongoing to this date [11-15]. The formal further development of weak turbulence theory was resumed by one of us (P.H.Y.) in recent years, starting from first principles and proceeding in a systematic way. Initially the formalism included only electrostatic oscillations, taking into account wave-wave and wave-particle interactions without considering discrete-particle effects [16]. The formalism was later expanded to include effects due to processes related to spontaneously emitted fluctuations, which come from discrete-particle effects, both for wave equations and for particle equations [17]. Further extension occurred when electromagnetic waves were incorporated into the formalism, initially without the discrete-particle effects [18], but later by incorporating effects representing spontaneously emitted thermal fluctuations $[19,20]$. The foundational equations that resulted from these developments were applied for actual applications, mostly related to quantitative analyses of nonlinear 
processes associated with the beam-plasma instability. Thus, numerical analyses were carried out in one or two dimensional systems, such as in Refs. [21-31].

The afore-mentioned formal developments on weak turbulence theory and numerical analyses thereof have not taken into account the collisional interaction between plasma particles. The reason had been that the time scale of the instability development, such as the growth of Langmuir waves due to the presence of a particle beam, as well as subsequent nonlinear three-wave decay and scattering processes, should be shorter than the time scale associated with binary collisional processes. However, in some of the applications that have been made, it was shown that nonlinear effects continue to operate far beyond the time scale of the instability saturation and nonlinear mode coupling, to the extent that an asymptotically steady state, or quasiequilibrium state, of the turbulent system becomes of relevance. Specifically, it was demonstrated that plasma in such a "turbulent equilibrium" state can be associated with a background of electromagnetic radiation $[30,32]$. It was also shown that the long-range, asymptotic state of the turbulence is associated with the inverse power-law velocity distribution function called the "kappa" distribution [25-27]. It is therefore pertinent to raise the question whether collisional processes can play a role in these processes of long-time evolution or not, even in tenuous turbulent systems.

In addition to the interest motivated by the above-mentioned theoretical conjecture and fundamental plasma physics issues, another salient point may be that there are space plasma phenomena whose explanation requires the presence of beams of particles under the influence of collisional processes. The prime example may be the emission of $\mathrm{x}$ rays via bremsstrahlung, by electrons traveling in the solar chromosphere [33-35]. There is evidence that suggests that the generation of Langmuir waves due to the presence of a beam may have influence on the velocity distribution of the x-raygenerating electrons [36], such that without properly taking the wave phenomena into account, the correct interpretation of the $\mathrm{x}$-ray spectra collected by spacecraft in the source region may not be achieved [37-39].

It is therefore deemed necessary that the long-time evolution of the beam-plasma system, and possibly other physical systems, may have to be reconsidered by taking into account not only the wave-particle and wave-wave interactions, but also the collisional interactions as well. In the literature, it is not difficult to find examples of studies that include collisional effects in the particle equation, but hardly any in conjunction with and within the context of the formalism of weak turbulence theory [40]. This is especially true if one is concerned with plasmas in tenuous space environments. For the equations that describe the evolution of the wave intensities, collisional effects are sometimes included, often in the form of a collisional damping term, but such a term is customarily introduced via heuristic arguments, instead of being derived from first principles of the kinetic theory [41].

These considerations motivate the present analysis, in which we start from first principles of kinetic theory and reformulate the standard weak turbulence theory, keeping contributions that arise from electrostatic fluctuations that are normally ignored in the literature. The approach to be used in the paper can be introduced as follows. The $N$-body probability distribution function in phase space $(\mathbf{r}, \mathbf{p})$, called the Klimontovich function, $N_{a}(\mathbf{r}, \mathbf{p}, t)$, defined by

$$
N_{a}(\mathbf{r}, \mathbf{p}, t)=\sum_{j=1}^{N} \delta\left[\mathbf{r}-\mathbf{r}_{j}^{a}(t)\right] \delta\left[\mathbf{p}-\mathbf{p}_{j}^{a}(t)\right],
$$

plays a central role in the kinetic theory of a classical manybody system. In the above $\mathbf{r}_{j}^{a}(t)$ and $\mathbf{p}_{j}^{a}(t)$ are exact particle orbits for the $j$ th particle of species $a, \mathbf{v}_{j}^{a}(t)=\dot{\mathbf{r}}_{j}^{a}(t), \dot{\mathbf{p}}_{j}^{a}(t)=$ $\mathbf{F}_{a}\left[\mathbf{r}_{j}^{a}(t), \mathbf{p}_{j}^{a}(t), t\right]$, where $\mathbf{F}_{a}(\mathbf{r}, \mathbf{p}, t)$ is the microscopic force that governs the interactions among the classical particles. For ionized gas, i.e., plasmas, $\mathbf{F}_{a}$ is, of course, the Lorentz force, $\mathbf{F}_{a}(\mathbf{r}, \mathbf{v}, t)=e_{a} \mathbf{E}(\mathbf{r}, t)+\left(e_{a} / c\right) \mathbf{v} \times \mathbf{B}(\mathbf{r}, t)$, where $a=e, i$ represents the electrons and ions, respectively, $e_{a}=-e$ for electrons and $e_{a}=e$ for ions (protons), $e$ being the unit electric charge, and $\mathbf{E}$ and $\mathbf{B}$ are electric and magnetic field vectors, respectively, satisfying Maxwell's equation. The governing equation that determines the time evolution of the Klimontovich function in phase space is equivalent to the Liouville equation for exact phase space mapping. Together with the Maxwell's equation, the fundamental equations for plasmas are thus given by

$$
\begin{gathered}
\left\{\frac{\partial}{\partial t}+\mathbf{v} \cdot \frac{\partial}{\partial \mathbf{r}}+e_{a}\left[\mathbf{E}(\mathbf{r}, t)+\frac{\mathbf{v}}{c} \mathbf{x} \mathbf{B}(\mathbf{r}, t)\right] \cdot \frac{\partial}{\partial \mathbf{p}}\right\} N_{a}(\mathbf{r}, \mathbf{p}, t)=0, \\
\frac{\partial}{\partial \mathbf{r}} \mathbf{x} \mathbf{E}(\mathbf{r}, t)+\frac{1}{c} \frac{\partial}{\partial t} \mathbf{B}(\mathbf{r}, t)=0, \\
\frac{\partial}{\partial \mathbf{r}} \mathbf{x} \mathbf{B}(\mathbf{r}, t)-\frac{1}{c} \frac{\partial}{\partial t} \mathbf{E}(\mathbf{r}, t)-\frac{4 \pi}{c} \sum_{a} e_{a} \int d \mathbf{p v} N_{a}(\mathbf{r}, \mathbf{p}, t)=0 .
\end{gathered}
$$

In Eq. (1.2), the other two equations of Maxwell's equations, $\nabla \cdot \mathbf{B}=0$ and $\nabla \cdot \mathbf{E}=4 \pi \sum_{a} e_{a} \int d \mathbf{p} N_{a}$ are implicit. The above exact dynamical equation for $N_{a}(\mathbf{r}, \mathbf{p}, t)$ contains no microscopic dissipation. The dissipations, be they collective or collisional, arise as a result of the loss of information from the ensemble average procedures.

The one-particle distribution function, $f_{a}(\mathbf{r}, \mathbf{p}, t)$, is the ensemble averaged Klimontovich function:

$$
f_{a}(\mathbf{r}, \mathbf{p}, t)=\left\langle N_{a}(\mathbf{r}, \mathbf{p}, t)\right\rangle .
$$

The Vlasov equation is formally identical to the first of Eqs. (1.2), except that the equation describes the dynamical path of the smoothed ensemble averaged function $f_{a}(\mathbf{r}, \mathbf{p}, t)$. For plasmas, the Vlasov-Maxwell equations are thus given by

$$
\begin{gathered}
\left\{\frac{\partial}{\partial t}+\mathbf{v} \cdot \frac{\partial}{\partial \mathbf{r}}+e_{a}\left[\mathbf{E}(\mathbf{r}, t)+\frac{\mathbf{v}}{c} \mathbf{x} \mathbf{B}(\mathbf{r}, t)\right] \cdot \frac{\partial}{\partial \mathbf{p}}\right\} f_{a}(\mathbf{r}, \mathbf{p}, t)=0 \\
\frac{\partial}{\partial \mathbf{r}} \mathbf{x} \mathbf{E}(\mathbf{r}, t)+\frac{1}{c} \frac{\partial}{\partial t} \mathbf{B}(\mathbf{r}, t)=0 \\
\frac{\partial}{\partial \mathbf{r}} \mathbf{x} \mathbf{B}(\mathbf{r}, t)-\frac{1}{c} \frac{\partial}{\partial t} \mathbf{E}(\mathbf{r}, t)-\frac{4 \pi}{c} \sum_{a} e_{a} \int d \mathbf{p v} f_{a}(\mathbf{r}, \mathbf{p}, t)=0 .
\end{gathered}
$$

The Klimontovich and Vlasov equations, (1.2) and (1.4), are mathematically identical. The only difference is the initial configuration. In the case of the Klimontovich equation, the initial condition contains the information on the discrete nature of the particles, $N_{a}(\mathbf{r}, \mathbf{p}, 0)=\sum_{j=1}^{N} \delta\left[\mathbf{r}-\mathbf{r}_{j}(0)\right] \delta\left[\mathbf{p}-\mathbf{p}_{j}(0)\right]$, 
as evidenced from singular delta function conditions. In contrast, the Vlasov equation acts upon the smoothed, or ensemble averaged one-particle initial distribution function, $f_{a}(\mathbf{r}, \mathbf{p}, 0)$.

It is the discrete particle property that determines the importance of binary collisions or spontaneous thermal fluctuations. The purely collisionless plasma corresponds to the limit of $g=0$, where $g$ is the plasma parameter defined by $g=1 /\left(\hat{n} \lambda_{D e}^{3}\right), \lambda_{D e}=\sqrt{T_{e} /\left(4 \pi \hat{n} e^{2}\right)}$ being the Debye length. Here $T_{e}$ is the electron temperature defined in the unit of energy and $\hat{n}$ is the ambient number density. For a finite $g$, the discrete particle effects become nonnegligible. Conceptually, the Vlasov equation describes the situation for $g=0$. The more general Klimontovich equation, on the other hand, is applicable for small but finite $g$, for which binary collisions and effects owing to spontaneous thermal fluctuations become nonnegligible. In the literature on collisional effects on the wave dynamics, sometimes the collisional operator is simply added to the right-hand side of Eq. (1.4) in an ad hoc manner [42],

$$
\begin{aligned}
& \left\{\frac{\partial}{\partial t}+\mathbf{v} \cdot \frac{\partial}{\partial \mathbf{r}}+e_{a}\left[\mathbf{E}(\mathbf{r}, t)+\frac{\mathbf{v}}{c} \times \mathbf{B}(\mathbf{r}, t)\right] \cdot \frac{\partial}{\partial \mathbf{p}}\right\} f_{a}(\mathbf{r}, \mathbf{p}, t) \\
& =\sum_{b} C_{a b}\left(f_{a}, f_{b}\right)
\end{aligned}
$$

where $\sum_{b} C_{a b}\left(f_{a}, f_{b}\right)$ represents the collision operator, and sometimes the discussion of collisional effects is made in a context in which nonlinear effects do not play effective role [40]. For numerical analysis of the relatively dense plasmas discussed in fusion research, collisional effects have been applied for decades in the context of quasilinear theory, by simply adding the collision operator to the right-hand of the equation for the time evolution of the particle distribution function [43-47]. It is also a common approximation in the literature to heuristically replace the collision operator by an effective collision frequency, $\sum_{b} C_{a b}\left(f_{a}, f_{b}\right)=-v_{\text {coll }} f_{a}$. With this approximated collision operator, upon linearization of the resulting equation and upon coupling the perturbed particle distribution function with the wave equation, the particle collision frequency then gets absorbed into the wave-particle resonance conditions, $\omega-\mathbf{k} \cdot \mathbf{v}-i v_{\text {coll }}=0$, and thereby the collisional damping rate is calculated. In short, in the standard heuristic treatment of the collisional effects on wave phenomena, the particle collision frequency automatically gets translated to the wave collisional damping rate.

However, the procedure found in the literature is not rigorous. The proper procedure is to derive the collision integral on the basis of the equation for exact phase space mapping, i.e., Eq. (1.2). The collisional integral should emerge only as a result of systematic statistical averaging procedure and upon introducing a hierarchy of correlations. Moreover, the collisional damping rate for plasma waves must be computed independently of the effective particle collision frequency. The purpose of the present paper is to discuss the correct way of incorporating the collisional dynamics in the plasma turbulence from the standpoint of first principles. Starting from the correct governing microscopic equation (1.2), namely, the Klimontovich equation with no ad hoc collision term on the right-hand side and having no microscopic dissipation, it will be shown that upon introducing a hierarchy of correlations and ensemble averages, a particle kinetic equation that contains both the collective effects, spontaneous thermal effects as well as collision integral emerges, while at the same time, the generalized wave kinetic equation that contains the collective linear and nonlinear wave-particle interaction terms, nonlinear wave-wave interaction terms, and collisional damping terms emerges.

The present paper is organized as follows. In Sec. II we present the basic formalism of weak turbulence theory, including discrete particle effects, and obtain formal kinetic equations for waves and particles. In Sec. III we discuss with further details the application of the basic formalism to the analysis of the nonlinear evolution of linear modes of plasma, discussing the different mechanisms which can be identified in the kinetic equations. In Sec. IV we present a detailed analysis on the extension of the weak turbulence formalism, which can be made by taking into account the effect of noneigenmodes of plasma, that is, the effect of fluctuations which do not satisfy the dispersion relations for normal modes of plasma, and show that these new effects lead to a collisional term in the particle equations, and to terms which can be identified as collisional damping and as emission of electrostatic waves by collisional processes, in the equation for the evolution of the spectral wave intensities. Section V is dedicated to final comments on the work which has been presented in the paper and on possible developments to be obtained with application of the formalism.

\section{WEAK TURBULENCE FORMALISM INCLUDING DISCRETE PARTICLE EFFECTS}

\section{A. Nonlinear plasma kinetic equation}

In what follows, let us assume that the plasma under consideration is immersed in a field-free environment, and the predominant mode of interaction is the electrostatic force. Under such a situation the basic equation (1.2) reduces to

$$
\left[\frac{\partial}{\partial t}+\mathbf{v} \cdot \frac{\partial}{\partial \mathbf{r}}+e_{a} \mathbf{E}(\mathbf{r}, t) \cdot \frac{\partial}{\partial \mathbf{p}}\right] N_{a}(\mathbf{r}, \mathbf{p}, t)=0, \quad \frac{\partial}{\partial \mathbf{r}} \cdot \mathbf{E}(\mathbf{r}, t)-4 \pi \sum_{a} e_{a} \int d \mathbf{p} N_{a}(\mathbf{r}, \mathbf{p}, t)=0
$$

It is useful to consider the Klimontovich function (1.1) describing the phase space evolution of free particles (ideal gas) that do not interact with each other,

$$
N_{a}^{0}(\mathbf{r}, \mathbf{p}, t)=\sum_{j=1}^{N} \delta\left[\mathbf{r}-\mathbf{r}_{j}^{a 0}(t)\right] \delta\left[\mathbf{p}-\mathbf{p}_{j}^{a 0}(t)\right]
$$


where $\mathbf{r}_{j}^{a 0}(t)$ and $\mathbf{p}_{j}^{a 0}(t)$ are exact orbits of free streaming particles satisfying $\dot{\mathbf{p}}_{j}^{a 0}(t)=0$ and $\mathbf{v}_{j}^{a 0}(t)=\dot{\mathbf{r}}_{j}^{a 0}(t)$. The corresponding Klimontovich equation is

$$
\left(\frac{\partial}{\partial t}+\mathbf{v} \cdot \frac{\partial}{\partial \mathbf{r}}\right) N_{a}^{0}(\mathbf{r}, \mathbf{p}, t)=0 .
$$

Let us denote the deviation of the Klimontovich function $N_{a}(\mathbf{r}, \mathbf{p}, t)$ from its average $f_{a}(\mathbf{r}, \mathbf{p}, t)=\left\langle N_{a}(\mathbf{r}, \mathbf{p}, t)\right\rangle$, that is, the fluctuation, $\delta N_{a}(\mathbf{r}, \mathbf{p}, t)$ :

$$
\delta N_{a}(\mathbf{r}, \mathbf{p}, t)=N_{a}(\mathbf{r}, \mathbf{p}, t)-\left\langle N_{a}(\mathbf{r}, \mathbf{p}, t)\right\rangle .
$$

Assuming that the random phase for the fluctuations the ensemble average of $\delta N_{a}(\mathbf{r}, \mathbf{p}, t)$ is zero, $\left\langle\delta N_{a}(\mathbf{r}, \mathbf{p}, t)\right\rangle=0$. Since the medium is free of average field, by definition, the electric field is made only of fluctuations, $\mathbf{E}(\mathbf{r}, t)=\delta \mathbf{E}(\mathbf{r}, t)$. Then Eq. $(2.1)$ can be reexpressed as

$$
\begin{array}{r}
\left(\frac{\partial}{\partial t}+\mathbf{v} \cdot \frac{\partial}{\partial \mathbf{r}}\right) f_{a}(\mathbf{r}, \mathbf{p}, t)+e_{a} \frac{\partial}{\partial \mathbf{p}} \cdot\left\langle\delta \mathbf{E}(\mathbf{r}, t) \delta N_{a}(\mathbf{r}, \mathbf{p}, t)\right\rangle=0 \\
\left(\frac{\partial}{\partial t}+\mathbf{v} \cdot \frac{\partial}{\partial \mathbf{r}}\right) \delta N_{a}(\mathbf{r}, \mathbf{p}, t)+e_{a} \delta \mathbf{E}(\mathbf{r}, t) \cdot \frac{\partial f_{a}(\mathbf{r}, \mathbf{p}, t)}{\partial \mathbf{p}}+e_{a} \frac{\partial}{\partial \mathbf{p}} \cdot\left[\delta \mathbf{E}(\mathbf{r}, t) \delta N_{a}(\mathbf{r}, \mathbf{p}, t)-\left\langle\delta \mathbf{E}(\mathbf{r}, t) \delta N_{a}(\mathbf{r}, \mathbf{p}, t)\right\rangle\right]=0 \\
\frac{\partial}{\partial \mathbf{r}} \cdot \delta \mathbf{E}(\mathbf{r}, t)-4 \pi \sum_{a} e_{a} \int d \mathbf{p} \delta N_{a}(\mathbf{r}, \mathbf{p}, t)=0 .
\end{array}
$$

We also define the fluctuation of the free streaming Klimontovich function:

$$
\delta N_{a}^{0}(\mathbf{r}, \mathbf{p}, t)=N_{a}^{0}(\mathbf{r}, \mathbf{p}, t)-\left\langle N_{a}^{0}(\mathbf{r}, \mathbf{p}, t)\right\rangle .
$$

Upon assuming that the ensemble average of the free streaming Klimontovich function is, in fact, the same as the one-particle distribution function,

$$
f_{a}(\mathbf{r}, \mathbf{p}, t)=\left\langle N_{a}^{0}(\mathbf{r}, \mathbf{p}, t)\right\rangle
$$

the equation for $\delta N_{a}^{0}(\mathbf{r}, \mathbf{p}, t)$ is simply given by

$$
\left(\frac{\partial}{\partial t}+\mathbf{v} \cdot \frac{\partial}{\partial \mathbf{r}}\right) \delta N_{a}^{0}(\mathbf{r}, \mathbf{p}, t)=0
$$

We may subtract Eq. (2.10) from the equation for $\delta N_{a}$ in Eq. (2.6), to obtain

$$
\left(\frac{\partial}{\partial t}+\mathbf{v} \cdot \frac{\partial}{\partial \mathbf{r}}\right)\left[\delta N_{a}(\mathbf{r}, \mathbf{p}, t)-\delta N_{a}^{0}(\mathbf{r}, \mathbf{p}, t)\right]+e_{a} \delta \mathbf{E}(\mathbf{r}, t) \cdot \frac{\partial f_{a}(\mathbf{r}, \mathbf{p}, t)}{\partial \mathbf{p}}+e_{a} \frac{\partial}{\partial \mathbf{p}} \cdot\left[\delta \mathbf{E}(\mathbf{r}, t) \delta N_{a}(\mathbf{r}, \mathbf{p}, t)-\left\langle\delta \mathbf{E}(\mathbf{r}, t) \delta N_{a}(\mathbf{r}, \mathbf{p}, t)\right\rangle\right]=0 .
$$

In Eq. (2.11) we are not interested in $\delta N_{a}^{0}(\mathbf{r}, \mathbf{p}, t)$ per se, but rather in the ensemble average of the product of two quantities $\delta N_{a}^{0}(\mathbf{r}, \mathbf{p}, t)$ and $\delta N_{a}^{0}\left(\mathbf{r}^{\prime}, \mathbf{p}^{\prime}, t^{\prime}\right)$, that is, the two-body correlation function for the fluctuations of the free-streaming Klimontovich function, $\left\langle\delta N_{a}^{0}(\mathbf{r}, \mathbf{p}, t) \delta N_{a}^{0}\left(\mathbf{r}^{\prime}, \mathbf{p}^{\prime}, t^{\prime}\right)\right\rangle$. We may compute this quantity directly from the definition (2.2), which is explicitly written as

$$
N_{a}^{0}(\mathbf{r}, \mathbf{p}, t)=\sum_{i=1}^{N} \delta\left(\mathbf{r}-\mathbf{v}_{i}^{a} t\right) \delta\left(\mathbf{p}-\mathbf{p}_{i}^{a}\right) .
$$

Note that one could include the initial phase space positions of the free-streaming particle position in the above definition, $N_{a}^{0}(\mathbf{r}, \mathbf{p}, t)=\sum_{i=1}^{N} \delta\left(\mathbf{r}-\mathbf{r}^{a 0}-\mathbf{v}_{i}^{a} t\right) \delta\left(\mathbf{p}-\mathbf{p}_{i}^{a}\right)$. However, in the end, the initial particle position becomes irrelevant, as we shall see shortly. Equation (2.12) is, thus, a convenient shortcut definition. From this we have

$$
\begin{aligned}
\left\langle\delta N_{a}^{0}(\mathbf{r}, \mathbf{p}, t) \delta N_{b}^{0}\left(\mathbf{r}^{\prime}, \mathbf{p}^{\prime}, t^{\prime}\right)\right\rangle= & \left\langle\left[N_{a}^{0}(\mathbf{r}, \mathbf{p}, t)-\left\langle N_{a}^{0}(\mathbf{r}, \mathbf{p}, t)\right\rangle\right]\left[N_{b}^{0}\left(\mathbf{r}^{\prime}, \mathbf{p}^{\prime}, t^{\prime}\right)-\left\langle N_{b}^{0}\left(\mathbf{r}^{\prime}, \mathbf{p}^{\prime}, t^{\prime}\right)\right\rangle\right]\right\rangle \\
= & \left\langle N_{a}^{0}(\mathbf{r}, \mathbf{p}, t) N_{b}^{0}\left(\mathbf{r}^{\prime}, \mathbf{p}^{\prime}, t^{\prime}\right)\right\rangle-\left\langle N_{a}^{0}(\mathbf{r}, \mathbf{p}, t)\left\langle N_{b}^{0}\left(\mathbf{r}^{\prime}, \mathbf{p}^{\prime}, t^{\prime}\right)\right\rangle\right\rangle \\
& -\left\langle\left\langle N_{a}^{0}(\mathbf{r}, \mathbf{p}, t)\right\rangle N_{b}^{0}\left(\mathbf{r}^{\prime}, \mathbf{p}^{\prime}, t^{\prime}\right)\right\rangle+\left\langle N_{a}^{0}(\mathbf{r}, \mathbf{p}, t)\right\rangle\left\langle N_{b}^{0}\left(\mathbf{r}^{\prime}, \mathbf{p}^{\prime}, t^{\prime}\right)\right\rangle \\
= & \left\langle N_{a}^{0}(\mathbf{r}, \mathbf{p}, t) N_{b}^{0}\left(\mathbf{r}^{\prime}, \mathbf{p}^{\prime}, t^{\prime}\right)\right\rangle-\left\langle N_{a}^{0}(\mathbf{r}, \mathbf{p}, t)\right\rangle\left\langle N_{b}^{0}\left(\mathbf{r}^{\prime}, \mathbf{p}^{\prime}, t^{\prime}\right)\right\rangle \\
= & \left\langle\sum_{i \neq j=1}^{N} \delta\left(\mathbf{r}-\mathbf{v}_{i}^{a} t\right) \delta\left(\mathbf{p}-\mathbf{p}_{i}^{a}\right) \delta\left(\mathbf{r}^{\prime}-\mathbf{v}_{j}^{b} t^{\prime}\right) \delta\left(\mathbf{p}^{\prime}-\mathbf{p}_{j}^{b}\right)\right\rangle \\
& +\delta_{a b} \delta\left(\mathbf{r}-\mathbf{r}^{\prime}-\mathbf{v} t+\mathbf{v}^{\prime} t^{\prime}\right) \delta\left(\mathbf{p}-\mathbf{p}^{\prime}\right)\left\langle\sum_{i=1}^{N} \delta\left(\mathbf{r}-\mathbf{v}_{i}^{a} t\right) \delta\left(\mathbf{p}-\mathbf{p}_{i}^{a}\right)\right\rangle-\left\langle N_{a}^{0}(\mathbf{r}, \mathbf{p}, t)\right\rangle\left\langle N_{b}^{0}\left(\mathbf{r}^{\prime}, \mathbf{p}^{\prime}, t^{\prime}\right)\right\rangle .
\end{aligned}
$$


Note that the inclusion of initial particle positions would not have made a difference since the delta-function condition $\delta_{a b}$ exactly cancels out the difference in the initial position vectors $\mathbf{r}^{a 0}-\mathbf{r}^{b 0}$. For free-streaming particles

$$
\left\langle\sum_{i \neq j=1}^{N} \delta\left(\mathbf{r}-\mathbf{v}_{i}^{a} t\right) \delta\left(\mathbf{p}-\mathbf{p}_{i}^{a}\right) \delta\left(\mathbf{r}^{\prime}-\mathbf{v}_{j}^{b} t^{\prime}\right) \delta\left(\mathbf{p}^{\prime}-\mathbf{p}_{j}^{b}\right)\right\rangle=\left\langle N_{a}^{0}(\mathbf{r}, \mathbf{p}, t)\right\rangle\left\langle N_{b}^{0}\left(\mathbf{r}^{\prime}, \mathbf{p}^{\prime}, t^{\prime}\right)\right\rangle .
$$

Thus we have

$$
\left\langle\delta N_{a}^{0}(\mathbf{r}, \mathbf{p}, t) \delta N_{b}^{0}\left(\mathbf{r}^{\prime}, \mathbf{p}^{\prime}, t^{\prime}\right)\right\rangle=\delta_{a b} \delta\left[\mathbf{r}-\mathbf{r}^{\prime}-\mathbf{v}\left(t-t^{\prime}\right)\right] \delta\left(\mathbf{p}-\mathbf{p}^{\prime}\right) f_{a}(\mathbf{r}, \mathbf{p}, t) .
$$

To summarize, the set of self-consistent equations, which forms the basis for nonlinear plasma turbulence theory including the effects of single-particle fluctuations, are given by the formal particle kinetic equation, i.e., the equation for $f_{a}(\mathbf{r}, \mathbf{p}, t)[\mathrm{Eq} .(2.5)]$, the equation for perturbed distribution function $\delta N_{a}(\mathbf{r}, \mathbf{p}, t)$ [Eq. (2.11)], the wave equation (2.7), and the definition (2.15). It is convenient to work in the spectral representation. We assume that the perturbed quantities are functions of two distinct time scales, the fast time scale of the fluctuations, $t^{\prime}$, and the slow time scale of the evolution of the distribution function and the wave amplitude, $t$. This means that we may express $\delta N_{a}(\mathbf{r}, \mathbf{p}, t) \rightarrow \delta N_{a}\left(\mathbf{r}, \mathbf{p}, t, t^{\prime}\right), \delta N_{a}^{0}(\mathbf{r}, \mathbf{p}, t) \rightarrow \delta N_{a}^{0}\left(\mathbf{r}, \mathbf{p}, t, t^{\prime}\right)$, and $\delta \mathbf{E}(\mathbf{r}, t) \rightarrow \delta \mathbf{E}\left(\mathbf{r}, t, t^{\prime}\right)$. We are henceforth interested in spatially uniform system, $f_{a}(\mathbf{r}, \mathbf{p}, t)=f_{a}(\mathbf{p}, t)$. We assume that the perturbations can be decomposed in the sense of the customary Fourier-Laplace transformation over the fast time scale of the fluctuations, $t^{\prime}$, while the amplitudes of the spectra vary in the slow time scale, $t$,

$$
\begin{aligned}
\delta N_{a}\left(\mathbf{r}, \mathbf{p}, t, t^{\prime}\right) & =\int d \mathbf{k} \int_{L} d \omega \delta N_{\mathbf{k} \omega}^{a}(\mathbf{p}, t) e^{i \mathbf{k} \cdot \mathbf{r}-i \omega t^{\prime}}, \quad \delta \mathbf{E}\left(\mathbf{r}, t, t^{\prime}\right)=\int d \mathbf{k} \int_{L} d \omega \delta \mathbf{E}_{\mathbf{k} \omega}(t) e^{i \mathbf{k} \cdot \mathbf{r}-i \omega t^{\prime}} \\
\delta N_{\mathbf{k} \omega}^{a}(\mathbf{p}, t) & =\frac{1}{(2 \pi)^{4}} \int d \mathbf{r} \int_{0}^{\infty} d t^{\prime} \delta N_{a}\left(\mathbf{r}, \mathbf{p}, t, t^{\prime}\right) e^{-i \mathbf{k} \cdot \mathbf{r}+i \omega t^{\prime}}, \quad \delta \mathbf{E}_{\mathbf{k} \omega}(t)=\frac{1}{(2 \pi)^{4}} \int d \mathbf{r} \int_{0}^{\infty} d t^{\prime} \delta \mathbf{E}\left(\mathbf{r}, t, t^{\prime}\right) e^{-i \mathbf{k} \cdot \mathbf{r}+i \omega t^{\prime}},
\end{aligned}
$$

where the integration $\int_{L} d \omega$ is taken along the path $L$ stretching from $\omega=-\infty+i \sigma$ to $\omega=\infty+i \sigma(\sigma>0$ and $\sigma \rightarrow 0)$. In the Fourier-Laplace transformation defined above, the time dependence of the spectral amplitudes $\delta N_{\mathbf{k} \omega}^{a}(\mathbf{v}, t)$ and $\delta \mathbf{E}_{\mathbf{k} \omega}(t)$ is assumed to be slow and adiabatic. These quantities are calculated as if they are independent of time on the fast wave scale $\left(t^{\prime} \sim \omega^{-1}\right)$. We also write the electrostatic field in terms of the potential:

$$
\delta \mathbf{E}_{\mathbf{k}, \omega}=-\frac{i \mathbf{k}}{|k|} \delta \phi_{\mathbf{k}, \omega}
$$

Then, in the two-time step approximation, the relevant equations are

$$
\begin{aligned}
& \frac{\partial f_{a}(\mathbf{p}, t)}{\partial t}=i e_{a} \int d \mathbf{k} \int d \omega \int d \mathbf{k}^{\prime} \int d \omega^{\prime} \mathbf{k}^{\prime} \cdot \frac{\partial}{\partial \mathbf{p}}\left\langle\delta \phi_{\mathbf{k}^{\prime}, \omega^{\prime}}(t) \delta N_{\mathbf{k}, \omega}^{a}(\mathbf{p}, t)\right) e^{i\left(\mathbf{k}+\mathbf{k}^{\prime}\right) \cdot \mathbf{r}-i\left(\omega+\omega^{\prime}\right) t^{\prime}}, \\
& \left(\omega-\mathbf{k} \cdot \mathbf{v}+i \frac{\partial}{\partial t}\right)\left[\delta N_{\mathbf{k}, \omega}^{a}(\mathbf{p}, t)-\delta N_{\mathbf{k}, \omega}^{a 0}(\mathbf{p}, t)\right]=-e_{a} \delta \phi_{\mathbf{k}, \omega}(t) \mathbf{k} \cdot \frac{\partial f_{a}(\mathbf{p}, t)}{\partial \mathbf{p}}-e_{a} \int d \mathbf{k}^{\prime} \int d \omega^{\prime} \mathbf{k}^{\prime} \\
& \cdot \frac{\partial}{\partial \mathbf{p}}\left[\delta \phi_{\mathbf{k}^{\prime}, \omega^{\prime}}(t) \delta N_{\mathbf{k}-\mathbf{k}^{\prime}, \omega-\omega^{\prime}}^{a}(\mathbf{p}, t)-\left\langle\delta \phi_{\mathbf{k}^{\prime}, \omega^{\prime}}(t) \delta N_{\mathbf{k}-\mathbf{k}^{\prime}, \omega-\omega^{\prime}}^{a}(\mathbf{p}, t)\right\rangle\right], \\
& \delta \phi_{\mathbf{k}, \omega}(t)=\sum_{a} \frac{4 \pi e_{a}}{k^{2}} \int d \mathbf{p} \delta N_{\mathbf{k}, \omega}^{a}(\mathbf{p}, t) .
\end{aligned}
$$

The Fourier transformation of the free streaming particle fluctuations, $\left\langle\delta N_{a}^{0}\left(\mathbf{r}, \mathbf{p}, t, t^{\prime}\right) \delta N_{b}^{0}\left(\mathbf{r}^{\prime}, \mathbf{p}^{\prime}, t, t^{\prime \prime}\right)\right\rangle$, in fast time scale $t^{\prime}$ is given by

$$
\begin{aligned}
\left\langle\delta N_{a}^{0}(\mathbf{p}, t) \delta N_{b}^{0}\left(\mathbf{p}^{\prime}, t\right)\right\rangle_{\mathbf{k}, \omega}= & \delta_{a b} \delta\left(\mathbf{p}-\mathbf{p}^{\prime}\right)(2 \pi)^{-4} \int_{0}^{\infty} d \tau e^{-i \mathbf{k} \cdot \mathbf{v} \tau+i \omega \tau-\Delta \tau} f_{a}(\mathbf{p}, t) \\
& +\delta_{a b} \delta\left(\mathbf{p}-\mathbf{p}^{\prime}\right)(2 \pi)^{-4} \int_{-\infty}^{0} d \tau e^{-i \mathbf{k} \cdot \mathbf{v} \tau+i \omega \tau+\Delta \tau} f_{a}(\mathbf{p}, t),
\end{aligned}
$$

where $\Delta \rightarrow 0^{+}$and $\tau=\left|t^{\prime}-t^{\prime \prime}\right|$. The above definition reduces to the customary Fourier transformation for $\Delta=0$ exactly. From this we have

$$
\left\langle\delta N_{a}^{0}(\mathbf{p}, t) \delta N_{b}^{0}\left(\mathbf{p}^{\prime}, t\right)\right\rangle_{\mathbf{k}, \omega}=\delta_{a b} \delta\left(\mathbf{p}-\mathbf{p}^{\prime}\right)(2 \pi)^{-3} \delta(\omega-\mathbf{k} \cdot \mathbf{v}) f_{a}(\mathbf{p}, t) .
$$

For stationary and homogeneous turbulence (which means that the correlation of two fluctuating quantities depend only the relative time and distance, not on absolute values; of course, such an assumption is violated when the underlying turbulence exhibits intermittent behavior), the cumulants of two fluctuating quantities $A(\mathbf{r}, t)$ and $B\left(\mathbf{r}, t^{\prime}\right)$ are a function of the relative distance and relative time only:

$$
\left\langle A(\mathbf{r}, t) B\left(\mathbf{r}, t^{\prime}\right)\right\rangle=\langle A B\rangle_{\mathbf{r}-\mathbf{r}^{\prime}, t-t^{\prime}} \equiv G\left(\mathbf{r}-\mathbf{r}^{\prime}, t-t^{\prime}\right)
$$


In spectral representation, this implies

$$
\langle A B\rangle_{\mathbf{k}, \omega}=\left\langle A_{\mathbf{k}, \omega} B_{-\mathbf{k},-\omega}\right\rangle,
$$

or more generally,

$$
\left\langle A_{\mathbf{k}, \omega} B_{\mathbf{k}^{\prime}, \omega^{\prime}}\right\rangle=\langle A B\rangle_{\mathbf{k}, \omega} \delta\left(\mathbf{k}+\mathbf{k}^{\prime}\right) \delta\left(\omega+\omega^{\prime}\right) .
$$

This simplifies the formal particle kinetic equation (2.18).

At this point, we make the approximation by formally absorbing the slow time derivatives into the definition for the angular frequency,

$$
\omega \rightarrow \omega+i \frac{\partial}{\partial t}
$$

and suppress the slow time $t$ henceforth, until we reintroduce it at an appropriate later stage. Employing the above approximations we now have a set of equations that forms the basis of subsequent weak turbulence analysis:

$$
\begin{aligned}
\frac{\partial f_{a}(\mathbf{v})}{\partial t}= & i e_{a} \int d \mathbf{k} \int d \omega \mathbf{k} \cdot \frac{\partial}{\partial \mathbf{p}}\left\langle\delta \phi_{\mathbf{k}, \omega} \delta N_{-\mathbf{k},-\omega}^{a}(\mathbf{p})\right\rangle \\
\delta N_{\mathbf{k}, \omega}^{a}(\mathbf{p})= & \delta N_{\mathbf{k}, \omega}^{a 0}(\mathbf{p})-\frac{e_{a}}{\omega-\mathbf{k} \cdot \mathbf{v}+i 0} \mathbf{k} \cdot \frac{\partial f_{a}(\mathbf{p})}{\partial \mathbf{p}} \delta \phi_{\mathbf{k}, \omega}-\frac{e_{a}}{\omega-\mathbf{k} \cdot \mathbf{v}+i 0} \\
& \times \int d \mathbf{k}^{\prime} \int d \omega^{\prime} \mathbf{k}^{\prime} \cdot \frac{\partial}{\partial \mathbf{p}}\left[\delta \phi_{\mathbf{k}^{\prime}, \omega^{\prime}} \delta N_{\mathbf{k}-\mathbf{k}^{\prime}, \omega-\omega^{\prime}}^{a}(\mathbf{p})-\left\langle\delta \phi_{\mathbf{k}^{\prime}, \omega^{\prime}} \delta N_{\mathbf{k}-\mathbf{k}^{\prime}, \omega-\omega^{\prime}}^{a}(\mathbf{p})\right\rangle\right] \\
\delta \phi_{\mathbf{k}, \omega}= & \sum_{a} \frac{4 \pi e_{a}}{k^{2} \int d \mathbf{p} \delta N_{\mathbf{k}, \omega}^{a}(\mathbf{p}),} \\
\left\langle\delta N_{a}^{0}(\mathbf{p}) \delta N_{b}^{0}\left(\mathbf{p}^{\prime}\right)\right\rangle_{\mathbf{k}, \omega}= & (2 \pi)^{-3} \delta_{a b} \delta\left(\mathbf{p}-\mathbf{p}^{\prime}\right) \delta(\omega-\mathbf{k} \cdot \mathbf{v}) f_{a}(\mathbf{p}) .
\end{aligned}
$$

Let us introduce the following short-hand notations:

$$
q \equiv(\mathbf{k}, \omega), \quad \mathbf{g}_{q}=\mathbf{g}_{\mathbf{k}, \omega}^{a}=-\frac{e_{a}}{\omega-\mathbf{k} \cdot \mathbf{v}+i 0} \frac{\partial}{\partial \mathbf{p}} .
$$

We also omit $\delta$ for the perturbed quantities. Then the nonlinear equation for the perturbed distribution can be expressed compactly as

$$
N_{q}^{a}=N_{q}^{a 0}+\mathbf{k} \cdot \mathbf{g}_{q} f_{a} \phi_{q}+\int d q^{\prime} \mathbf{k}^{\prime} \cdot \mathbf{g}_{q}\left[\phi_{q^{\prime}} N_{q-q^{\prime}}^{a}-\left\langle\phi_{q^{\prime}} N_{q-q^{\prime}}^{a}\right\rangle\right]
$$

\section{B. Iterative solution of nonlinear equation for perturbed distribution}

To solve the nonlinear equation for $N_{q}^{a}$, we employ iterative means,

$$
N_{q}^{a}=N_{q}^{a(1)}+N_{q}^{a(2)}+N_{q}^{a(3)}+\cdots,
$$

under the assumption that each order in the perturbative expansion is of the similar magnitude with the electric field perturbation of the same order:

$$
N_{q}^{a(n)} \propto \phi_{q}^{n}
$$

Under this assumption, let us write the iterative solution order by order. Then we have to the third order:

$$
N_{q}^{a(1)}=N_{q}^{a 0}+\mathbf{k} \cdot \mathbf{g}_{q} f_{a} \phi_{q}, \quad N_{q}^{a(2)}=\int d q^{\prime} \mathbf{k}^{\prime} \cdot \mathbf{g}_{q}\left[\phi_{q^{\prime}} N_{q-q^{\prime}}^{a(1)}-\left\langle\phi_{q^{\prime}} N_{q-q^{\prime}}^{a(1)}\right\rangle, \quad N_{q}^{a(3)}=\int d q^{\prime} \mathbf{k}^{\prime} \cdot \mathbf{g}_{q}\left[\phi_{q^{\prime}} N_{q-q^{\prime}}^{a(2)}-\left\langle\phi_{q^{\prime}} N_{q-q^{\prime}}^{a(2)}\right\rangle\right] .\right.
$$

The truncation of the iterative solution up to third order (or for that matter, any finite order) implies that the perturbation expansion in electric field wave amplitude is valid. This assumption is one of the key ingredients of the weak turbulence theory where the wave energy density is assumed to be sufficiently lower than the particle thermal energy. For a highly turbulent system, the perturbation expansion fails. Theories that attempt to partially sum the infinite series such as Eq. (2.33) are known as the renormalized kinetic theory. The present paper does not discuss such a theory. For interested readers, see, e.g., a recent monograph by Diamond et al. [48].

The effects of single particles are embodied by the term $N_{q}^{a 0}$ in the equation for $N_{q}^{a(1)}$. This term will be treated as a correction term to the collective term $\mathbf{k} \cdot \mathbf{g}_{q} f_{a} \phi_{q}$. This will be justified a posteriori by the fact various terms in the particle and wave kinetic equations that owe their existence to the single-particle effects turn out to be proportional to the so-called plasma parameter $g=1 /\left(\hat{n} \lambda_{D e}^{3}\right)$ (the inverse of the number of plasma particles within a sphere with radius equal to the Debye length), which is 
generally very small for plasmas. Consequently, we may ignore nonlinear terms that arise from $N_{q}^{a 0}$ term. An iterative solution is thus given by

$$
\begin{aligned}
& N_{q}^{a(1)}=N_{q}^{a 0}+\mathbf{k} \cdot \mathbf{g}_{q} f_{a} \phi_{q}, \\
& N_{q}^{a(2)}=\int d q^{\prime} \mathbf{k}^{\prime} \cdot \mathbf{g}_{q}\left(\mathbf{k}-\mathbf{k}^{\prime}\right) \cdot \mathbf{g}_{q-q^{\prime}} f_{a}\left[\phi_{q^{\prime}} \phi_{q-q^{\prime}}-\left\langle\phi_{q^{\prime}} \phi_{q-q^{\prime}}\right\rangle\right] \\
& N_{q}^{a(3)}=\int d q^{\prime} \int d q^{\prime \prime} \mathbf{k}^{\prime} \cdot \mathbf{g}_{q}^{a} \mathbf{k}^{\prime \prime} \cdot \mathbf{g}_{q-q^{\prime}}\left(\mathbf{k}-\mathbf{k}^{\prime}-\mathbf{k}^{\prime \prime}\right) \cdot \mathbf{g}_{q-q^{\prime}-q^{\prime \prime}} f_{a}\left[\phi_{q^{\prime}} \phi_{q^{\prime \prime}} \phi_{q-q^{\prime}-q^{\prime \prime}}-\phi_{q^{\prime}}\left\langle\phi_{q^{\prime \prime}} \phi_{q-q^{\prime}-q^{\prime \prime}}\right\rangle-\left\langle\phi_{q^{\prime}} \phi_{q^{\prime \prime}} \phi_{q-q^{\prime}-q^{\prime \prime}}\right\rangle\right]
\end{aligned}
$$

We may add the results to obtain the net solution. However, we symmetrize the final expression with respect to the dummy integral variable:

$$
\begin{aligned}
N_{q}^{a}= & N_{q}^{a 0}+\alpha_{a}(q) f_{a} \phi_{q}+\sum_{\substack{q_{1} \\
\left(q_{1}+q_{2}=q\right)}} \sum_{q_{2}} \alpha_{a}^{(2)}\left(q_{1} \mid q_{2}\right) f_{a}\left[\phi_{q_{1}} \phi_{q_{2}}-\left\langle\phi_{q_{1}} \phi_{q_{2}}\right\rangle\right] \\
& +\sum_{\substack{q_{1} \\
\left(q_{1}+q_{2}+q_{3}=q\right)}} \sum_{q_{3}} \alpha_{a}^{(3)}\left(q_{1}\left|q_{2}\right| q_{3}\right) f_{a}\left[\phi_{q_{1}} \phi_{q_{2}} \phi_{q_{3}}-\phi_{q_{1}}\left\langle\phi_{q_{2}} \phi_{q_{3}}\right\rangle-\left\langle\phi_{q_{1}} \phi_{q_{2}} \phi_{q_{3}}\right\rangle\right], \\
\alpha_{a}(q)= & \mathbf{k} \cdot \mathbf{g}_{q}, \\
\alpha_{a}^{(2)}\left(q_{1} \mid q_{2}\right)= & \frac{1}{2}\left[\left(\mathbf{k}_{1} \cdot \mathbf{g}_{q_{1}+q_{2}}\right)\left(\mathbf{k}_{2} \cdot \mathbf{g}_{q_{2}}\right)+\left(\mathbf{k}_{2} \cdot \mathbf{g}_{q_{1}+q_{2}}\right)\left(\mathbf{k}_{1} \cdot \mathbf{g}_{q_{1}}\right)\right], \\
\alpha_{a}^{(3)}\left(q_{1}\left|q_{2}\right| q_{3}\right)= & \frac{1}{2}\left(\mathbf{k}_{1} \cdot \mathbf{g}_{q_{1}+q_{2}+q_{3}}\right)\left[\left(\mathbf{k}_{2} \cdot \mathbf{g}_{q_{2}+q_{3}}\right)\left(\mathbf{k}_{3} \cdot \mathbf{g}_{q_{3}}\right)+\left(\mathbf{k}_{3} \cdot \mathbf{g}_{q_{2}+q_{3}}\right)\left(\mathbf{k}_{2} \cdot \mathbf{g}_{q_{2}}\right)\right] .
\end{aligned}
$$

Let us now combine the solution (2.37) and the perturbed Poisson's equation (2.29):

$$
\begin{aligned}
\phi_{q}= & \sum_{a} \frac{4 \pi e_{a}}{k^{2}} \int d \mathbf{p}\left\{N_{q}^{a 0}(\mathbf{p})+\alpha_{a}(q) f_{a}(\mathbf{p}) \phi_{q}+\sum_{\substack{q_{1} \\
\left(q_{1}+q_{2}=q\right)}} \sum_{q_{2}} \alpha_{a}^{(2)}\left(q_{1} \mid q_{2}\right) f_{a}(\mathbf{p})\left[\phi_{q_{1}} \phi_{q_{2}}-\left\langle\phi_{q_{1}} \phi_{q_{2}}\right\rangle\right]\right. \\
& \left.+\sum_{\substack{q_{1} \\
\left(q_{1}+q_{2}+q_{3}=q\right)}} \sum_{q_{2}} \sum_{q_{3}} \alpha_{a}^{(3)}\left(q_{1}\left|q_{2}\right| q_{3}\right) f_{a}(\mathbf{p})\left[\phi_{q_{1}} \phi_{q_{2}} \phi_{q_{3}}-\phi_{q_{1}}\left\langle\phi_{q_{2}} \phi_{q_{3}}\right\rangle-\left\langle\phi_{q_{1}} \phi_{q_{2}} \phi_{q_{3}}\right\rangle\right]\right\} .
\end{aligned}
$$

We next define the various response functions for each species,

$$
\begin{aligned}
\chi_{a}(q) & =-\frac{4 \pi e_{a}}{k^{2}} \int d \mathbf{p} \alpha_{a}(q) f_{a}(\mathbf{p})=\frac{4 \pi e_{a}^{2}}{k^{2}} \int d \mathbf{p} \frac{\mathbf{k} \cdot \partial f_{a} / \partial \mathbf{p}}{\omega-\mathbf{k} \cdot \mathbf{v}+i 0}, \\
\chi_{a}^{(2)}\left(q_{1} \mid q_{2}\right) & =-\frac{4 \pi i e_{a}}{k_{1} k_{2}\left|\mathbf{k}_{1}+\mathbf{k}_{2}\right|} \int d \mathbf{p} \alpha_{a}^{(2)}\left(q_{1} \mid q_{2}\right) f_{a}(\mathbf{p}) \\
& =\frac{-i e_{a}}{2} \frac{4 \pi e_{a}^{2}}{k_{1} k_{2}\left|\mathbf{k}_{1}+\mathbf{k}_{2}\right|} \int d \mathbf{p} \frac{1}{\omega_{1}+\omega_{2}-\left(\mathbf{k}_{1}+\mathbf{k}_{2}\right) \cdot \mathbf{v}+i 0}\left[\mathbf{k}_{1} \cdot \frac{\partial}{\partial \mathbf{p}}\left(\frac{\mathbf{k}_{2} \cdot \partial f_{a} / \partial \mathbf{p}}{\omega_{2}-\mathbf{k}_{2} \cdot \mathbf{v}+i 0}\right)+\mathbf{k}_{2} \cdot \frac{\partial}{\partial \mathbf{p}}\left(\frac{\mathbf{k}_{1} \cdot \partial f_{a} / \partial \mathbf{p}}{\omega_{1}-\mathbf{k}_{1} \cdot \mathbf{v}+i 0}\right)\right],
\end{aligned}
$$

$$
\begin{aligned}
\bar{\chi}_{a}^{(3)}\left(q_{1}\left|q_{2}\right| q_{3}\right)= & \frac{4 \pi e_{a}}{k_{1} k_{2} k_{3}\left|\mathbf{k}_{1}+\mathbf{k}_{2}+\mathbf{k}_{3}\right|} \int d \mathbf{p} \alpha_{a}^{(3)}\left(q_{1}\left|q_{2}\right| q_{3}\right) f_{a}(\mathbf{p}) \\
= & \frac{(-i)^{2} e_{a}^{2}}{2} \frac{4 \pi e_{a}^{2}}{k_{1} k_{2} k_{3}\left|\mathbf{k}_{1}+\mathbf{k}_{2}+\mathbf{k}_{3}\right|} \int d \mathbf{p} \frac{1}{\omega_{1}+\omega_{2}+\omega_{3}-\left(\mathbf{k}_{1}+\mathbf{k}_{2}+\mathbf{k}_{3}\right) \cdot \mathbf{v}+i 0} \\
& \times \mathbf{k}_{1} \cdot \frac{\partial}{\partial \mathbf{p}}\left\{\frac{1}{\omega_{2}+\omega_{3}-\left(\mathbf{k}_{2}+\mathbf{k}_{3}\right) \cdot \mathbf{v}+i 0}\left[\mathbf{k}_{2} \cdot \frac{\partial}{\partial \mathbf{p}}\left(\frac{\mathbf{k}_{3} \cdot \partial f_{a} / \partial \mathbf{p}}{\omega_{3}-\mathbf{k}_{3} \cdot \mathbf{v}+i 0}\right)+\mathbf{k}_{3} \cdot \frac{\partial}{\partial \mathbf{p}}\left(\frac{\mathbf{k}_{2} \cdot \partial f_{a} / \partial \mathbf{p}}{\omega_{2}-\mathbf{k}_{2} \cdot \mathbf{v}+i 0}\right)\right]\right\},
\end{aligned}
$$

and the total susceptibilities by summing over the particle species:

$$
\chi(q)=\sum_{a} \chi_{a}(q), \quad \epsilon(q)=1+\chi(q), \quad \chi^{(2)}\left(q_{1} \mid q_{2}\right)=\sum_{a} \chi_{a}^{(2)}\left(q_{1} \mid q_{2}\right), \quad \bar{\chi}^{(3)}\left(q_{1}\left|q_{2}\right| q_{3}\right)=\sum_{a} \bar{\chi}_{a}^{(3)}\left(q_{1}\left|q_{2}\right| q_{3}\right)
$$


The definitions and notations of the various dielectric susceptibilities are consistent with Ref. [9]. Then Eq. (2.38) may be reexpressed as

$$
\begin{aligned}
0= & k^{2} \epsilon(q) \phi_{q}-\sum_{\substack{q_{1} \\
\left(q_{1}+q_{2}=q\right)}} \sum_{q_{2}} i k_{1} k_{2} k \chi^{(2)}\left(q_{1} \mid q_{2}\right)\left[\phi_{q_{1}} \phi_{q_{2}}-\left\langle\phi_{q_{1}} \phi_{q_{2}}\right\rangle\right] \\
& -\sum_{\substack{q_{1} \\
\left(q_{1}+q_{2}+q_{3}=q\right)}} \sum_{\substack{q_{3} \\
q_{1}}} k_{1} k_{2} k_{3} k \bar{\chi}^{(3)}\left(q_{1}\left|q_{2}\right| q_{3}\right)\left[\phi_{q_{1}} \phi_{q_{2}} \phi_{q_{3}}-\phi_{q_{1}}\left|\phi_{q_{2}} \phi_{q_{3}}\right\rangle-\left\langle\phi_{q_{1}} \phi_{q_{2}} \phi_{q_{3}}\right\rangle\right]-\sum_{a} 4 \pi e_{a} \int d \mathbf{p} N_{q}^{a 0}(\mathbf{p}) .
\end{aligned}
$$

Multiplying $\phi_{-q}$ to Eq. (2.43), and taking the ensemble average, we have

$$
\begin{aligned}
0= & k^{2} \epsilon(q)\left\langle\phi^{2}\right\rangle_{q}-i \sum_{q^{\prime}} k k^{\prime}\left|\mathbf{k}-\mathbf{k}^{\prime}\right| \chi^{(2)}\left(q^{\prime} \mid q-q^{\prime}\right)\left\langle\phi_{q^{\prime}} \phi_{q-q^{\prime}} \phi_{-q}\right\rangle \\
& -2 \sum_{q^{\prime}} k^{2} k^{\prime 2} \bar{\chi}^{(3)}\left(q^{\prime}\left|-q^{\prime}\right| q\right)\left\langle\phi^{2}\right\rangle_{q^{\prime}}\left\langle\phi^{2}\right\rangle_{q}-\sum_{a} 4 \pi e_{a} \int d \mathbf{p}\left\langle\phi_{-q} N_{q}^{a 0}(\mathbf{p})\right\rangle .
\end{aligned}
$$

Next, let us multiply $N_{-q}^{a 0}(\mathbf{p})$ to Eq. (2.43) and take the average. Then we replace $q$ by $-q$ to obtain

$$
\begin{aligned}
k^{2} \epsilon(-q)\left\langle\phi_{-q} N_{q}^{a 0}(\mathbf{p})\right\rangle= & -i \sum_{q^{\prime}} k k^{\prime}\left|\mathbf{k}-\mathbf{k}^{\prime}\right| \chi^{(2) *}\left(q^{\prime} \mid q-q^{\prime}\right)\left\langle\phi_{-q^{\prime}} \phi_{-q+q^{\prime}} N_{q}^{a 0}(\mathbf{p})\right\rangle \\
& +2 \sum_{q^{\prime}} k^{2} k^{\prime 2} \bar{\chi}^{(3) *}\left(q^{\prime}\left|-q^{\prime}\right| q\right)\left\langle\phi^{2}\right\rangle_{q^{\prime}}\left\langle\phi_{-q} N_{q}^{a 0}(\mathbf{p})\right\rangle+\sum_{b} 4 \pi e_{b} \int d \mathbf{p}^{\prime}\left\langle N_{-q}^{b 0}\left(\mathbf{p}^{\prime}\right) N_{q}^{a 0}(\mathbf{p})\right\rangle .
\end{aligned}
$$

We make use of Eq. (2.30) and the property $\epsilon(-q)=\epsilon^{*}(q)$ to arrive at

$$
\begin{aligned}
\left\langle\phi_{-q} N_{q}^{a 0}(\mathbf{p})\right\rangle= & \frac{4 \pi e_{a}}{(2 \pi)^{3} k^{2} \epsilon^{*}(q)} \delta(\omega-\mathbf{k} \cdot \mathbf{v}) f_{a}(\mathbf{p})+\frac{2}{\epsilon^{*}(q)} \sum_{q^{\prime}} k^{\prime 2} \bar{\chi}^{(3) *}\left(q^{\prime}\left|-q^{\prime}\right| q\right)\left\langle\phi^{2}\right\rangle_{q^{\prime}}\left\langle\phi_{-q} N_{q}^{a 0}(\mathbf{p})\right\rangle \\
& -\frac{i}{k \epsilon^{*}(q)} \sum_{q^{\prime}} k^{\prime}\left|\mathbf{k}-\mathbf{k}^{\prime}\right| \chi^{(2) *}\left(q^{\prime} \mid q-q^{\prime}\right)\left\langle\phi_{-q^{\prime}} \phi_{-q+q^{\prime}} N_{q}^{a 0}(\mathbf{p})\right\rangle .
\end{aligned}
$$

Note that the term $\left\langle\phi_{-q} N_{q}^{a 0}(\mathbf{p})\right\rangle$ appears on both sides of Eq. (2.46). Since the quantity $\left\langle\phi_{-q} N_{q}^{a 0}(\mathbf{p})\right\rangle$ that appears on the right-hand side can be treated as a small correction to the linear solution, we may ignore such a term. Consequently, inserting Eq. (2.46) to the right-hand side of Eq. (2.44), we arrive at

$$
\begin{aligned}
0= & \epsilon(q)\left\langle E^{2}\right\rangle_{q}-i \int d q^{\prime} \chi^{(2)}\left(q^{\prime} \mid q-q^{\prime}\right) k k^{\prime}\left|\mathbf{k}-\mathbf{k}^{\prime}\right|\left\langle\phi_{q^{\prime}} \phi_{q-q^{\prime}} \phi_{-q}\right\rangle-2 \int d q^{\prime} \bar{\chi}^{(3)}\left(q^{\prime}\left|-q^{\prime}\right| q\right)\left\langle E^{2}\right\rangle_{q^{\prime}}\left\langle E^{2}\right\rangle_{q} \\
& -\sum_{a} \int d \mathbf{p} \frac{\left(4 \pi e_{a}\right)^{2}}{(2 \pi)^{3} k^{2} \epsilon^{*}(q)} \delta(\omega-\mathbf{k} \cdot \mathbf{v}) f_{a}(\mathbf{p})+i \sum_{a} \int d q^{\prime} \int d \mathbf{p} \frac{\left(4 \pi e_{a}\right) \chi^{(2) *}\left(q^{\prime} \mid q-q^{\prime}\right)}{k \epsilon^{*}(q)} k^{\prime}\left|\mathbf{k}-\mathbf{k}^{\prime}\right|\left\langle\phi_{-q^{\prime}} \phi_{-q+q^{\prime}} N_{q}^{a 0}(\mathbf{p})\right\rangle,
\end{aligned}
$$

where we have made use of

$$
\left\langle E^{2}\right\rangle_{q}=\left\langle k^{2} \phi^{2}\right\rangle_{q}
$$

Note that we use summation and integral over $q=(\mathbf{k}, \omega)$ interchangeably in the present paper, that is, $\sum_{q}=\int d q=\int d \mathbf{k} \int d \omega$.

\section{Three-body cumulants and nonlinear spectral balance equation}

Equation (2.47) shows that we need to obtain third-order cumulants, $\left\langle\phi_{q^{\prime}} \phi_{q-q^{\prime}} \phi_{-q}\right\rangle$, and $\left\langle\phi_{-q^{\prime}} \phi_{-q+q^{\prime}} N_{q}^{a 0}(\mathbf{p})\right\rangle$. We may construct these quantities from Eq. (2.43) by ignoring the third-order nonlinearity at the outset. We first note that the three-body cumulants are zero if nonlinear terms are neglected, since the linear eigenmodes are essentially plane wave solutions such that odd moments of the wave amplitudes vanish upon taking the ensemble average. Consequently, if we write the perturbed field as a sum of the plane-wave solution that satisfies the linear wave equation plus the correction owing to nonlinear terms, $\phi_{q}=\phi_{q}^{(0)}+\phi_{q}^{(1)}$, where $\phi_{q}^{(0)}$ satisfies the linear wave equation, $\epsilon(q) \phi_{q}^{(0)}=0$, then upon inserting this expression in Eq. (2.43) and retaining the lowest-order terms within the nonlinear terms on the right-hand side, we obtain

$$
\phi_{q_{1}}^{(1)}=\frac{1}{k_{1}^{2} \epsilon\left(q_{1}\right)} \sum_{q^{\prime \prime}} i k_{1} k^{\prime \prime}\left|\mathbf{k}_{1}-\mathbf{k}^{\prime \prime}\right| \chi^{(2)}\left(q^{\prime \prime} \mid q_{1}-q^{\prime \prime}\right)\left[\phi_{q^{\prime \prime}}^{(0)} \phi_{q_{1}-q^{\prime \prime}}^{(0)}-\left\langle\phi_{q^{\prime \prime}}^{(0)} \phi_{q_{1}-q^{\prime \prime}}^{(0)}\right]\right]+\frac{1}{k_{1}^{2} \epsilon\left(q_{1}\right)} \sum_{a} 4 \pi e_{a} \int d \mathbf{v} N_{q_{1}}^{a 0}(\mathbf{p})
$$


The quantity $\left\langle\phi_{q^{\prime}} \phi_{q-q^{\prime}} \phi_{-q}\right\rangle$ can be constructed by successively making use of Eq. (2.49) for each of $\phi_{q^{\prime}}, \phi_{q-q^{\prime}}$, and $\phi_{-q}$,

$$
\left\langle\phi_{q^{\prime}} \phi_{q-q^{\prime}} \phi_{-q}\right\rangle=\left\langle\phi_{q^{\prime}}^{(1)} \phi_{q-q^{\prime}}^{(0)} \phi_{-q}^{(0)}\right\rangle+\left\langle\phi_{q^{\prime}}^{(0)} \phi_{q-q^{\prime}}^{(1)} \phi_{-q}^{(0)}\right\rangle+\left\langle\phi_{q^{\prime}}^{(0)} \phi_{q-q^{\prime}}^{(0)} \phi_{-q}^{(1)}\right\rangle+\cdots .
$$

After inserting Eqs. (2.49) to (2.50) we omit the superscript (0) on the right-hand side to obtain

$$
\begin{aligned}
\left\langle\phi_{q^{\prime}} \phi_{q-q^{\prime}} \phi_{-q}\right\rangle= & \sum_{q^{\prime \prime}} \frac{i k^{\prime} k^{\prime \prime}\left|\mathbf{k}^{\prime}-\mathbf{k}^{\prime \prime}\right| \chi^{(2)}\left(q^{\prime \prime} \mid q^{\prime}-q^{\prime \prime}\right)}{k^{\prime 2} \epsilon\left(q^{\prime}\right)}\left[\left\langle\phi_{q^{\prime \prime}} \phi_{q^{\prime}-q^{\prime \prime}} \phi_{q-q^{\prime}} \phi_{-q}\right\rangle-\left\langle\phi_{q^{\prime \prime}} \phi_{q^{\prime}-q^{\prime \prime}}\right\rangle\left\langle\phi_{q-q^{\prime}} \phi_{-q}\right\rangle\right] \\
& +\sum_{q^{\prime \prime}} \frac{i\left|\mathbf{k}-\mathbf{k}^{\prime}\right| k^{\prime \prime}\left|\mathbf{k}-\mathbf{k}^{\prime}-\mathbf{k}^{\prime \prime}\right| \chi^{(2)}\left(q^{\prime \prime} \mid q-q^{\prime}-q^{\prime \prime}\right)}{\left|\mathbf{k}-\mathbf{k}^{\prime}\right|^{2} \epsilon\left(q-q^{\prime}\right)}\left[\left\langle\phi_{q^{\prime \prime}} \phi_{q-q^{\prime}-q^{\prime \prime}} \phi_{q^{\prime}} \phi_{-q}\right\rangle-\left\langle\phi_{q^{\prime \prime}} \phi_{q-q^{\prime}-q^{\prime \prime}}\right\rangle\left\langle\phi_{q^{\prime}} \phi_{-q}\right\rangle\right] \\
& +\sum_{q^{\prime \prime}} \frac{i k k^{\prime \prime}\left|\mathbf{k}+\mathbf{k}^{\prime \prime}\right| \chi^{(2)}\left(q^{\prime \prime} \mid-q-q^{\prime \prime}\right)}{k^{2} \epsilon^{*}(q)}\left[\left\langle\phi_{q^{\prime \prime}} \phi_{-q-q^{\prime \prime}} \phi_{q^{\prime}} \phi_{q-q^{\prime}}\right\rangle-\left\langle\phi_{q^{\prime \prime}} \phi_{-q-q^{\prime \prime}}\right\rangle\left\langle\phi_{q^{\prime}} \phi_{q-q^{\prime}}\right\rangle\right] \\
& +\sum_{a} 4 \pi e_{a} \int d \mathbf{p}\left[\frac{\left\langle N_{q^{\prime}}^{a 0}(\mathbf{p}) \phi_{q-q^{\prime}} \phi_{-q}\right\rangle}{k^{\prime 2} \epsilon\left(q^{\prime}\right)}+\frac{\left\langle N_{q-q^{\prime}}^{a 0}(\mathbf{p}) \phi_{q^{\prime}} \phi_{-q}\right\rangle}{\left|\mathbf{k}-\mathbf{k}^{\prime}\right|^{2} \epsilon\left(q-q^{\prime}\right)}+\frac{\left\langle N_{-q}^{a 0}(\mathbf{p}) \phi_{q^{\prime}} \phi_{q-q^{\prime}}\right\rangle}{k^{2} \epsilon^{*}(q)}\right] .
\end{aligned}
$$

Making use of the symmetry property,

$$
\chi^{(2)}\left(-q_{1} \mid-q_{2}\right)=-\chi^{(2) *}\left(q_{1} \mid q_{2}\right),
$$

and decomposing the four-body cumulants as products of two-body cumulants while ignoring irreducible components, thereby closing the hierarchy of correlations,

$$
\begin{aligned}
\left\langle\phi_{q_{1}} \phi_{q_{2}} \phi_{q_{3}} \phi_{q_{4}}\right\rangle= & \delta\left(q_{1}+q_{2}+q_{3}+q_{4}\right)\left[\left\langle\phi_{q_{1}} \phi_{q_{2}}\right\rangle\left\langle\phi_{q_{3}} \phi_{q_{4}}\right\rangle \delta\left(q_{1}+q_{2}\right)\right. \\
& \left.+\left\langle\phi_{q_{1}} \phi_{q_{3}}\right\rangle\left\langle\phi_{q_{2}} \phi_{q_{4}}\right\rangle \delta\left(q_{1}+q_{3}\right)+\left\langle\phi_{q_{1}} \phi_{q_{4}}\right\rangle\left\langle\phi_{q_{2}} \phi_{q_{3}}\right\rangle \delta\left(q_{1}+q_{4}\right)\right],
\end{aligned}
$$

we obtain

$$
\begin{aligned}
\left\langle\phi_{q^{\prime}} \phi_{q-q^{\prime}} \phi_{-q}\right\rangle= & 2 i k k^{\prime}\left|\mathbf{k}-\mathbf{k}^{\prime}\right|\left[\frac{\chi^{(2)}\left(q \mid-q+q^{\prime}\right)}{k^{\prime 2} \epsilon\left(q^{\prime}\right)}\left\langle\phi^{2}\right\rangle_{q-q^{\prime}}\left\langle\phi^{2}\right\rangle_{q}+\frac{\chi^{(2)}\left(q \mid-q^{\prime}\right)}{\left|\mathbf{k}-\mathbf{k}^{\prime}\right|^{2} \epsilon\left(q-q^{\prime}\right)}\left\langle\phi^{2}\right\rangle_{q^{\prime}}\left\langle\phi^{2}\right\rangle_{q}-\frac{\chi^{(2) *}\left(q^{\prime} \mid q-q^{\prime}\right)}{k^{2} \epsilon^{*}(q)}\left\langle\phi^{2}\right\rangle_{q^{\prime}}\left\langle\phi^{2}\right\rangle_{q-q^{\prime}}\right] \\
& +\sum_{a} 4 \pi e_{a} \int d \mathbf{p}\left[\frac{\left\langle\phi_{q-q^{\prime}} \phi_{-q} N_{q^{\prime}}^{a 0}(\mathbf{p})\right\rangle}{k^{\prime 2} \epsilon\left(q^{\prime}\right)}+\frac{\left\langle\phi_{q^{\prime}} \phi_{-q} N_{q-q^{\prime}}^{a 0}(\mathbf{p})\right\rangle}{\left|\mathbf{k}-\mathbf{k}^{\prime}\right|^{2} \epsilon\left(q-q^{\prime}\right)}+\frac{\left\langle\phi_{q^{\prime}} \phi_{q-q^{\prime}} N_{-q}^{a 0}(\mathbf{p})\right\rangle}{k^{2} \epsilon^{*}(q)}\right] .
\end{aligned}
$$

The closure scheme introduced in Eq. (2.53), namely, ignoring the irreducible four-body cumulant, is the simplest closure, which in the theory of neutral fluid turbulence, is known as the quasinormal closure [49].

Let us insert Eq. (2.54) to Eq. (2.47). The result is

$$
\begin{aligned}
0= & \epsilon(q)\left\langle E^{2}\right\rangle_{q}-\sum_{a} \frac{\left(4 \pi e_{a}\right)^{2}}{(2 \pi)^{3} k^{2} \epsilon^{*}(q)} \int d \mathbf{p} \delta(\omega-\mathbf{k} \cdot \mathbf{v}) f_{a}(\mathbf{p})+2 \int d q^{\prime}\left\{\chi ^ { ( 2 ) } ( q ^ { \prime } | q - q ^ { \prime } ) \left[\frac{\chi^{(2)}\left(q \mid-q+q^{\prime}\right)}{\epsilon\left(q^{\prime}\right)}\left\langle E^{2}\right\rangle_{q-q^{\prime}}\right.\right. \\
& \left.\left.+\frac{\chi^{(2)}\left(q \mid-q^{\prime}\right)}{\epsilon\left(q-q^{\prime}\right)}\left\langle E^{2}\right\rangle_{q^{\prime}}\right]-\bar{\chi}^{(3)}\left(q^{\prime}\left|-q^{\prime}\right| q\right)\left\langle E^{2}\right\rangle_{q^{\prime}}\right\}\left\langle E^{2}\right\rangle_{q}-2 \int d q^{\prime} \frac{\left|\chi^{(2)}\left(q^{\prime} \mid q-q^{\prime}\right)\right|^{2}}{\epsilon^{*}(q)}\left\langle E^{2}\right\rangle_{q^{\prime}}\left\langle E^{2}\right\rangle_{q-q^{\prime}} \\
& -i \sum_{a} 4 \pi e_{a} \int d q^{\prime} \int d \mathbf{p} k k^{\prime}\left|\mathbf{k}-\mathbf{k}^{\prime}\right|\left\{\chi ^ { ( 2 ) } ( q ^ { \prime } | q - q ^ { \prime } ) \left[\frac{\left\langle\phi_{q-q^{\prime}} \phi_{-q} N_{q^{\prime}}^{a 0}(\mathbf{v})\right\rangle}{k^{\prime 2} \epsilon\left(q^{\prime}\right)}\right.\right. \\
& \left.\left.+\frac{\left\langle\phi_{q^{\prime}} \phi_{-q} N_{q-q^{\prime}}^{a 0}(\mathbf{p})\right\rangle}{\left|\mathbf{k}-\mathbf{k}^{\prime}\right|^{2} \epsilon\left(q-q^{\prime}\right)}+\frac{\left\langle\phi_{q^{\prime}} \phi_{q-q^{\prime}} N_{-q}^{a 0}(\mathbf{p})\right\rangle}{k^{2} \epsilon^{*}(q)}\right]-\chi^{(2) *}\left(q^{\prime} \mid q-q^{\prime}\right) \frac{\left\langle\phi_{-q^{\prime}} \phi_{-q+q^{\prime}} N_{q}^{a 0}(\mathbf{p})\right\rangle}{k^{2} \epsilon^{*}(q)}\right\}
\end{aligned}
$$

where we have made use of Eq. (2.48). The above shows that we need to evaluate the remaining third-order cumulants $\left\langle\phi_{q-q^{\prime}} \phi_{-q} N_{q^{\prime}}^{a 0}(\mathbf{p})\right\rangle,\left\langle\phi_{q^{\prime}} \phi_{-q} N_{q-q^{\prime}}^{a 0}(\mathbf{p})\right\rangle,\left\langle\phi_{q^{\prime}} \phi_{q-q^{\prime}} N_{-q}^{a 0}(\mathbf{p})\right\rangle$, and $\left\langle\phi_{-q^{\prime}} \phi_{-q+q^{\prime}} N_{q}^{a 0}(\mathbf{p})\right\rangle$. These quantities are but special cases of a generic form $\left\langle\phi_{q_{1}} \phi_{-q_{1}+q_{2}} N_{-q_{2}}^{a 0}(\mathbf{p})\right\rangle$. Let us proceed to evaluate this quantity. Making use of Eq. (2.49) to evaluate $\phi_{q_{1}}$ and $\phi_{-q_{1}+q_{2}}$ successively, we have

$$
\left\langle\phi_{q_{1}} \phi_{-q_{1}+q_{2}} N_{-q_{2}}^{a 0}(\mathbf{p})\right\rangle=\frac{8 \pi e_{a} i}{(2 \pi)^{3} k_{1} k_{2}\left|\mathbf{k}_{1}-\mathbf{k}_{2}\right| \epsilon\left(q_{2}\right)}\left[\frac{\chi^{(2)}\left(q_{2} \mid q_{1}-q_{2}\right)}{\epsilon\left(q_{1}\right)}\left\langle E^{2}\right\rangle_{q_{1}-q_{2}}+\frac{\chi^{(2)}\left(-q_{1} \mid q_{2}\right)}{\epsilon\left(-q_{1}+q_{2}\right)}\left\langle E^{2}\right\rangle_{q_{1}}\right] \delta\left(\omega_{2}-\mathbf{k}_{2} \cdot \mathbf{v}\right) f_{a}(\mathbf{p}) .
$$

Identifying $q_{1}=q-q^{\prime}$ and $q_{2}=-q^{\prime}$, we may obtain the expression for $\left\langle\phi_{q-q^{\prime}} \phi_{-q} N_{q^{\prime}}^{a 0}(\mathbf{p})\right\rangle$. Making the identification for $q_{1}=q^{\prime}$ and $q_{2}=-q+q^{\prime}$, we may also have $\left\langle\phi_{q^{\prime}} \phi_{-q} N_{q-q^{\prime}}^{a 0}(\mathbf{v})\right\rangle$. Likewise, setting $q_{1}=q^{\prime}$ and $q_{2}=q$ leads to $\left\langle\phi_{q^{\prime}} \phi_{q-q^{\prime}} N_{-q}^{a 0}(\mathbf{v})\right\rangle$. Finally, 
identifying $q_{1}=-q^{\prime}$ and $q_{2}=-q$ yields the expression for $\left\langle\phi_{-q^{\prime}} \phi_{-q+q^{\prime}} N_{q}^{a 0}(\mathbf{v})\right\rangle$. In this way, the contributions from all the necessary third-order cumulants to Eq. (2.55) can be obtained.

After some tedious but otherwise straightforward algebraic manipulations, one may derive the nonlinear spectral balance equation,

$$
\begin{aligned}
0= & \epsilon(q)\left\langle E^{2}\right\rangle_{q}-\sum_{a} \frac{\left(4 \pi e_{a}\right)^{2}}{(2 \pi)^{3} k^{2} \epsilon^{*}(q)} \int d \mathbf{p} \delta(\omega-\mathbf{k} \cdot \mathbf{v}) f_{a}(\mathbf{p})-2 \int d q^{\prime} \frac{\left|\chi^{(2)}\left(q^{\prime} \mid q-q^{\prime}\right)\right|^{2}}{\epsilon^{*}(q)}\left\langle E^{2}\right\rangle_{q^{\prime}}\left\langle E^{2}\right\rangle_{q-q^{\prime}} \\
& +2 \int d q^{\prime}\left[\left\{\chi^{(2)}\left(q^{\prime} \mid q-q^{\prime}\right)\right\}^{2}\left(\frac{\left\langle E^{2}\right\rangle_{q-q^{\prime}}}{\epsilon\left(q^{\prime}\right)}+\frac{\left\langle E^{2}\right\rangle_{q^{\prime}}}{\epsilon\left(q-q^{\prime}\right)}\right)-\bar{\chi}^{(3)}\left(q^{\prime}\left|-q^{\prime}\right| q\right)\left\langle E^{2}\right\rangle_{q^{\prime}}\right]\left\langle E^{2}\right\rangle_{q} \\
& +\sum_{a} \int d q^{\prime} \frac{2\left(4 \pi e_{a}\right)^{2}}{(2 \pi)^{3} k^{\prime 2}\left|\epsilon\left(q^{\prime}\right)\right|^{2}}\left[\frac{\left\{\chi^{(2)}\left(q^{\prime} \mid q-q^{\prime}\right)\right\}^{2}}{\epsilon\left(q-q^{\prime}\right)}\left\langle E^{2}\right\rangle_{q}-\frac{\left|\chi^{(2)}\left(q^{\prime} \mid q-q^{\prime}\right)\right|^{2}}{\epsilon^{*}(q)}\left\langle E^{2}\right\rangle_{q-q^{\prime}}\right] \int d \mathbf{p} \delta\left(\omega^{\prime}-\mathbf{k}^{\prime} \cdot \mathbf{v}\right) f_{a}(\mathbf{p}) \\
& +\sum_{a} \int d q^{\prime} \frac{2\left(4 \pi e_{a}\right)^{2}}{(2 \pi)^{3}\left|\mathbf{k}-\mathbf{k}^{\prime}\right|^{2}\left|\epsilon\left(q-q^{\prime}\right)\right|^{2}}\left[\frac{\left\{\chi^{(2)}\left(q^{\prime} \mid q-q^{\prime}\right)\right\}^{2}}{\epsilon\left(q^{\prime}\right)}\left\langle E^{2}\right\rangle_{q}\right. \\
& \left.-\frac{\left|\chi^{(2)}\left(q^{\prime} \mid q-q^{\prime}\right)\right|^{2}}{\epsilon^{*}(q)}\left\langle E^{2}\right\rangle_{q^{\prime}}\right] \int d \mathbf{p} \delta\left[\omega-\omega^{\prime}-\left(\mathbf{k}-\mathbf{k}^{\prime}\right) \cdot \mathbf{v}\right] f_{a}(\mathbf{p}) .
\end{aligned}
$$

\section{Formal nonlinear wave kinetic equation}

At this point, we reintroduce the slow-time derivative, which was formally absorbed into the definition for new angular frequency in Eq. (2.26). Such a procedure was an approximate and heuristic treatment since the angular frequency $\omega$ appears in Eq. (2.19) in many places without the slow-time derivative. In fact, the only place where $\omega$ appears with the slow-time derivative factor $i \partial / \partial t$ is on the left-hand side of the equation for perturbed distribution. Consequently, when we extract the slow-time derivative from the angular frequency, we should be careful to implement it only in the leading term, namely, the linear response function:

$$
\epsilon(q)\left\langle E^{2}\right\rangle_{q} \rightarrow \epsilon\left(\mathbf{k}, \omega+i \frac{\partial}{\partial t}\right)\left\langle E^{2}\right\rangle_{q} \rightarrow\left[\epsilon(q)+\frac{i}{2} \frac{\partial \epsilon(q)}{\partial \omega} \frac{\partial}{\partial t}\right]\left\langle E^{2}\right\rangle_{q} .
$$

More rigorous method is to employ mathematical multiple time-scale perturbation analysis, as discussed in Ref. [5]. The present paper adopts the above heuristic approach. The reason for factor $1 / 2$ above is because the time derivative $\partial / \partial t$ is supposed to operate only on $E_{q}$ within the ensemble average $\left\langle E_{q}^{2}\right\rangle=\left\langle E_{q} E_{-q}\right\rangle$, but since both $E_{q}$ and $E_{-q}$ are affected by $\partial / \partial t$, we simply divided the net result by the factor 2 . This leads to

$$
\begin{aligned}
0= & \frac{i}{2} \frac{\partial \operatorname{Re} \epsilon(\mathbf{k}, \omega)}{\partial \omega} \frac{\partial\left\langle\delta E^{2}\right\rangle_{\mathbf{k}, \omega}}{\partial t}+\operatorname{Re} \epsilon(\mathbf{k}, \omega)\left\langle\delta E^{2}\right\rangle_{\mathbf{k}, \omega}+i \operatorname{Im} \epsilon(\mathbf{k}, \omega)\left\langle\delta E^{2}\right\rangle_{\mathbf{k}, \omega} \\
& +2 \int d \mathbf{k}^{\prime} \int d \omega^{\prime}\left[\left\{\chi^{(2)}\left(\mathbf{k}^{\prime}, \omega^{\prime} \mid \mathbf{k}-\mathbf{k}^{\prime}, \omega-\omega^{\prime}\right)\right\}^{2}\left\{\frac{\left\langle\delta E^{2}\right\rangle_{\mathbf{k}-\mathbf{k}^{\prime}, \omega-\omega^{\prime}}}{\epsilon\left(\mathbf{k}^{\prime}, \omega^{\prime}\right)}+\frac{\left\langle\delta E^{2}\right\rangle_{\mathbf{k}^{\prime}, \omega^{\prime}}}{\epsilon\left(\mathbf{k}-\mathbf{k}^{\prime}, \omega-\omega^{\prime}\right)}\right\}\right. \\
& \left.-\bar{\chi}^{(3)}\left(\mathbf{k}^{\prime}, \omega^{\prime}\left|-\mathbf{k}^{\prime},-\omega^{\prime}\right| \mathbf{k}, \omega\right)\left\langle\delta E^{2}\right\rangle_{\mathbf{k}^{\prime}, \omega^{\prime}}\right]\left\langle\delta E^{2}\right\rangle_{\mathbf{k}, \omega} \\
& -2 \int d \mathbf{k}^{\prime} \int d \omega^{\prime} \frac{\left|\chi^{(2)}\left(\mathbf{k}^{\prime}, \omega^{\prime} \mid \mathbf{k}-\mathbf{k}^{\prime}, \omega-\omega^{\prime}\right)\right|^{2}}{\epsilon^{*}(\mathbf{k}, \omega)}\left\langle\delta E^{2}\right\rangle_{\mathbf{k}^{\prime}, \omega^{\prime}}\left\langle\delta E^{2}\right\rangle_{\mathbf{k}-\mathbf{k}^{\prime}, \omega-\omega^{\prime}} \\
& -\frac{2}{\pi} \frac{1}{k^{2} \epsilon^{*}(\mathbf{k}, \omega)} \sum_{a} e_{a}^{2} \int d \mathbf{p} \delta(\omega-\mathbf{k} \cdot \mathbf{v}) f_{a}(\mathbf{p}) \\
& +\frac{4}{\pi} \int d \mathbf{k}^{\prime} \int d \omega^{\prime} \frac{1}{k^{\prime 2}\left|\epsilon\left(\mathbf{k}^{\prime}, \omega^{\prime}\right)\right|^{2}}\left[\frac{\left\{\chi^{(2)}\left(\mathbf{k}^{\prime}, \omega^{\prime} \mid \mathbf{k}-\mathbf{k}^{\prime}, \omega-\omega^{\prime}\right)\right\}^{2}}{\epsilon\left(\mathbf{k}-\mathbf{k}^{\prime}, \omega-\omega^{\prime}\right)}\left\langle\delta E^{2}\right\rangle_{\mathbf{k}, \omega}\right. \\
& \left.-\frac{\left|\chi^{(2)}\left(\mathbf{k}^{\prime}, \omega^{\prime} \mid \mathbf{k}-\mathbf{k}^{\prime}, \omega-\omega^{\prime}\right)\right|^{2}}{\epsilon^{*}(\mathbf{k}, \omega)}\left\langle\delta E^{2}\right\rangle_{\mathbf{k}-\mathbf{k}^{\prime}, \omega-\omega^{\prime}}\right] \sum_{a} e_{a}^{2} \int d \mathbf{p} \delta\left(\omega^{\prime}-\mathbf{k}^{\prime} \cdot \mathbf{v}\right) f_{a}(\mathbf{p}) \\
& +\frac{4}{\pi} \int d \mathbf{k}^{\prime} \int d \omega^{\prime} \frac{1}{\left|\mathbf{k}-\mathbf{k}^{\prime}\right|^{2}\left|\epsilon\left(\mathbf{k}-\mathbf{k}^{\prime}, \omega-\omega^{\prime}\right)\right|^{2}}\left[\frac{\left\{\chi^{(2)}\left(\mathbf{k}^{\prime}, \omega^{\prime} \mid \mathbf{k}-\mathbf{k}^{\prime}, \omega-\omega^{\prime}\right)\right\}^{2}}{\epsilon\left(\mathbf{k}^{\prime}, \omega^{\prime}\right)}\left\langle\delta E^{2}\right\rangle_{\mathbf{k}, \omega}\right. \\
& \left.-\frac{\left|\chi^{(2)}\left(\mathbf{k}^{\prime}, \omega^{\prime} \mid \mathbf{k}-\mathbf{k}^{\prime}, \omega-\omega^{\prime}\right)\right|^{2}}{\epsilon^{*}(\mathbf{k}, \omega)}\left\langle\delta E^{2}\right\rangle_{\mathbf{k}^{\prime}, \omega^{\prime}}\right] \sum_{a} e_{a}^{2} \int d \mathbf{p} \delta\left[\omega-\omega^{\prime}-\left(\mathbf{k}-\mathbf{k}^{\prime}\right) \cdot \mathbf{v}\right] f_{a}(\mathbf{p}),
\end{aligned}
$$

where we have resorted back to the long-hand notation. 


\section{E. Formal particle kinetic equation}

From Eq. (2.27), the particle kinetic equation is given by

$$
\frac{\partial f_{a}}{\partial t}=-i e_{a} \int d \mathbf{k} \int d \omega \mathbf{k} \cdot \frac{\partial}{\partial \mathbf{p}}\left\langle\phi_{-q} N_{q}^{a}\right\rangle .
$$

For the particle kinetic equation, it is sufficient to retain only the linear solution for $N_{q}^{a}$, that is, $N_{q}^{a}=N_{q}^{a 0}+\alpha_{a}(q) f_{a} \phi_{q}$; see Eq. (2.37). It is possible to retain corrections to the particle kinetic equation. In fact, Refs. [7,50] considered just such a problem, but they found that the nonlinear modification only leads to a small correction to the velocity-space diffusion coefficient. Substituting this to the quantity $\left\langle\phi_{-q} N_{q}^{a}\right\rangle$ on the right-hand side of Eq. (2.60), we have

$$
\left\langle\phi_{-q} N_{q}^{a}\right\rangle=\frac{1}{k^{2}} \alpha_{a}(q)\left\langle E^{2}\right\rangle_{q} f_{a}+\frac{4 \pi e_{a}}{(2 \pi)^{3} k^{2} \epsilon^{*}(q)} \delta(\omega-\mathbf{k} \cdot \mathbf{v}) f_{a}=-\frac{e_{a}\left\langle E^{2}\right\rangle_{q}}{\omega-\mathbf{k} \cdot \mathbf{v}+i 0} \frac{\mathbf{k}}{k^{2}} \cdot \frac{\partial f_{a}}{\partial \mathbf{p}}+\frac{4 \pi e_{a}}{(2 \pi)^{3} k^{2} \epsilon^{*}(q)} \delta(\omega-\mathbf{k} \cdot \mathbf{v}) f_{a} .
$$

This results in the desired formal particle kinetic equation:

$$
\frac{\partial f_{a}}{\partial t}=\pi e_{a}^{2} \int d \mathbf{k} \int d \omega\left(\frac{\mathbf{k}}{k} \cdot \frac{\partial}{\partial \mathbf{p}}\right) \delta(\omega-\mathbf{k} \cdot \mathbf{v})\left[\operatorname{Im} \frac{1}{2 \pi^{3} k \epsilon^{*}(\mathbf{k}, \omega)} f_{a}+\left\langle\delta E^{2}\right\rangle_{\mathbf{k}, \omega}\left(\frac{\mathbf{k}}{k} \cdot \frac{\partial f_{a}}{\partial \mathbf{p}}\right)\right] .
$$

Formal particle kinetic equation (2.62) together with the formal nonlinear wave kinetic equation (2.59) provide the basis of subsequent analysis of traditional weak turbulence theory that includes contributions from the linear eigenmodes only, as well as the more general weak turbulence theory for collisional plasmas that takes into account the additional contributions from fluctuations that are not characterized by the linear dispersion relations, i.e., the noneigenmode contributions.

\section{CUSTOMARY WEAK TURBULENCE THEORY FOR LINEAR EIGENMODES}

The customary weak turbulence theory found in the literature is tantamount to taking only the contributions from the linear eigenmodes. The basic idea is the following: the general electrostatic fluctuations $\left\langle E^{2}\right\rangle_{\mathbf{k}, \omega}$ are characterized by all $\mathbf{k}$ and $\omega$. However, in the customary theory, the assumption is made such that only those $\omega$ that satisfy the dispersion relation, $\omega=\omega_{\mathbf{k}}$ are important so that fluctuations characterizing noneigemodes, $\omega \neq \omega_{\mathbf{k}}$, are ignored. The starting point is Eq. (2.59). The imaginary part of Eq. (2.59) leads to the wave kinetic equation. The real part of the same equation leads to the wave dispersion relations. The real part of Eq. (2.59) has linear and nonlinear terms, as well as terms associated with discrete-particle effects. The discrete-particle terms in Eq. (2.59) are those terms associated with $f_{a}(\mathbf{p})$, and these terms will eventually be responsible for various spontaneous emission and scattering process. In the customary approach, as far as the real part of Eq. (2.59) is concerned, nonlinear terms and spontaneous emission terms are ignored, thus leading to

$$
\operatorname{Re} \epsilon(\mathbf{k}, \omega)\left\langle E^{2}\right\rangle_{\mathbf{k}, \omega}=0 .
$$

If one does not ignore the nonlinear terms in the real part of Eq. (2.59), then one obtains a theory either of nonlinear frequency shift of linear eigenmodes [51] or of nonlinear eigenmodes [52]. These will not be discussed here. We denote the dispersion relation $\operatorname{Re} \epsilon\left(\mathbf{k}, \omega_{\mathbf{k}}^{\alpha}\right)=0$, so that we may express the electric field fluctuation in terms of the eigenmode intensity by

$$
\left\langle\delta E^{2}\right\rangle_{\mathbf{k} \omega}=\sum_{\sigma= \pm 1} \sum_{\alpha=L, S} I_{\mathbf{k}}^{\sigma \alpha} \delta\left(\omega-\sigma \omega_{\mathbf{k}}^{\alpha}\right)
$$

where $I_{\mathbf{k}}^{\sigma \alpha}$ is the intensity for each eigenmode, $\alpha=L, S$ denotes the eigenmode or normal mode of the plasma, namely, Langmuir and ion-sound (or ion-acoustic) modes, respectively, whose respective dispersion relations are given by

$$
\omega_{\mathbf{k}}^{L}=\omega_{p e}\left(1+\frac{3}{2} k^{2} \lambda_{D e}^{2}\right), \quad \omega_{\mathbf{k}}^{S}=\sqrt{\frac{m_{e}}{m_{i}}} \omega_{p e} k \lambda_{D e} \frac{\left(1+3 T_{i} / T_{e}\right)^{1 / 2}}{\left(1+k^{2} \lambda_{D e}^{2}\right)^{1 / 2}},
$$

where $\omega_{p e}=\left(4 \pi \hat{n} e^{2} / m_{e}\right)^{1 / 2}$ is the plasma frequency, $\lambda_{D e}$ is the Debye length introduced earlier, $T_{i}$ and $T_{e}$ are ion and electron temperatures, respectively, and $m_{e}$ and $m_{i}$ are electron and proton masses, respectively. Then the imaginary part of Eq. (2.59) can be manipulated to yield

$$
\begin{aligned}
0= & \sum_{\sigma= \pm 1} \sum_{\alpha}\left[\frac{\partial \operatorname{Re} \epsilon\left(\mathbf{k}, \sigma \omega_{\mathbf{k}}^{\alpha}\right)}{\partial\left(\sigma \omega_{\mathbf{k}}^{\alpha}\right)} \frac{\partial I_{\mathbf{k}}^{\sigma \alpha}}{\partial t}+2 \operatorname{Im} \epsilon\left(\mathbf{k}, \sigma \omega_{\mathbf{k}}^{\alpha}\right) I_{\mathbf{k}}^{\sigma \alpha}\right] \delta\left(\omega-\sigma \omega_{\mathbf{k}}^{\alpha}\right) \\
& +\sum_{\sigma= \pm 1} \sum_{\alpha} 4 \operatorname{Im} \int d \mathbf{k}^{\prime}\left[\sum_{\sigma^{\prime \prime}= \pm 1} \sum_{\gamma} \frac{\left\{\chi^{(2)}\left(\mathbf{k}^{\prime}, \sigma \omega_{\mathbf{k}}^{\alpha}-\sigma^{\prime \prime} \omega_{\mathbf{k}-\mathbf{k}^{\prime}}^{\gamma} \mid \mathbf{k}-\mathbf{k}^{\prime}, \sigma^{\prime \prime} \omega_{\mathbf{k}-\mathbf{k}^{\prime}}^{\gamma}\right)\right\}^{2}}{\epsilon\left(\mathbf{k}^{\prime}, \sigma \omega_{\mathbf{k}}^{\alpha}-\sigma^{\prime \prime} \omega_{\mathbf{k}-\mathbf{k}^{\prime}}^{\gamma}\right)} I_{\mathbf{k}-\mathbf{k}^{\prime}}^{\sigma^{\prime \prime} \gamma}\right. \\
& +\sum_{\sigma^{\prime}= \pm 1} \sum_{\beta} \frac{\left\{\chi^{(2)}\left(\mathbf{k}^{\prime}, \sigma^{\prime} \omega_{\mathbf{k}^{\prime}}^{\beta} \mid \mathbf{k}-\mathbf{k}^{\prime}, \sigma \omega_{\mathbf{k}}^{\alpha}-\sigma^{\prime} \omega_{\mathbf{k}^{\prime}}^{\beta}\right)\right\}^{2}}{\epsilon\left(\mathbf{k}-\mathbf{k}^{\prime}, \sigma \omega_{\mathbf{k}}^{\alpha}-\sigma^{\prime} \omega_{\mathbf{k}^{\prime}}^{\beta}\right)} I_{\mathbf{k}^{\prime}}^{\sigma^{\prime} \beta}
\end{aligned}
$$




$$
\begin{aligned}
& \left.-\sum_{\sigma^{\prime}= \pm 1} \sum_{\beta} \bar{\chi}^{(3)}\left(\mathbf{k}^{\prime}, \sigma^{\prime} \omega_{\mathbf{k}^{\prime}}^{\beta}\left|-\mathbf{k}^{\prime},-\sigma^{\prime} \omega_{\mathbf{k}^{\prime}}^{\beta}\right| \mathbf{k}, \sigma \omega_{\mathbf{k}}^{\alpha}\right) I_{\mathbf{k}^{\prime}}^{\sigma^{\prime} \beta}\right] I_{\mathbf{k}}^{\sigma \alpha} \delta\left(\omega-\sigma \omega_{\mathbf{k}}^{\alpha}\right) \\
& -\sum_{\sigma^{\prime}, \sigma^{\prime \prime}= \pm 1} \sum_{\beta, \gamma} 4 \operatorname{Im} \int d \mathbf{k}^{\prime} \frac{\left|\chi^{(2)}\left(\mathbf{k}^{\prime}, \sigma^{\prime} \omega_{\mathbf{k}^{\prime}}^{\beta} \mid \mathbf{k}-\mathbf{k}^{\prime}, \sigma^{\prime \prime} \omega_{\mathbf{k}-\mathbf{k}^{\prime}}^{\gamma}\right)\right|^{2}}{\epsilon^{*}\left(\mathbf{k}, \sigma^{\prime} \omega_{\mathbf{k}^{\prime}}^{\beta}+\sigma^{\prime \prime} \omega_{\mathbf{k}-\mathbf{k}^{\prime}}^{\gamma}\right.} I_{\mathbf{k}^{\prime} \beta}^{\sigma^{\prime} \beta} I_{\mathbf{k}-\mathbf{k}^{\prime}}^{\sigma^{\prime \prime} \gamma} \delta\left(\omega-\sigma^{\prime} \omega_{\mathbf{k}^{\prime}}^{\beta}-\sigma^{\prime \prime} \omega_{\mathbf{k}-\mathbf{k}^{\prime}}^{\gamma}\right) \\
& -\operatorname{Im} \frac{4}{\pi k^{2} \epsilon^{*}(\mathbf{k}, \omega)} \sum_{a} e_{a}^{2} \int d \mathbf{p} \delta(\omega-\mathbf{k} \cdot \mathbf{v}) f_{a}(\mathbf{p}) \\
& +2 \operatorname{Im} \sum_{a} \int d \mathbf{k}^{\prime} d \omega^{\prime} \frac{2\left(4 \pi e_{a}\right)^{2}}{(2 \pi)^{3} k^{\prime 2}\left|\epsilon\left(\mathbf{k}^{\prime}, \omega^{\prime}\right)\right|^{2}}\left[\sum_{\sigma= \pm 1} \sum_{\alpha} \frac{\left\{\chi^{(2)}\left(\mathbf{k}^{\prime}, \omega^{\prime} \mid \mathbf{k}-\mathbf{k}^{\prime}, \sigma \omega_{\mathbf{k}}^{\alpha}-\omega^{\prime}\right)\right\}^{2}}{\epsilon\left(\mathbf{k}-\mathbf{k}^{\prime}, \sigma \omega_{\mathbf{k}}^{\alpha}-\omega^{\prime}\right)} I_{\mathbf{k}}^{\sigma \alpha} \delta\left(\omega-\sigma \omega_{\mathbf{k}}^{\alpha}\right)\right. \\
& \left.-\sum_{\sigma^{\prime \prime}= \pm 1} \sum_{\gamma} \frac{\left|\chi^{(2)}\left(\mathbf{k}^{\prime}, \omega^{\prime} \mid \mathbf{k}-\mathbf{k}^{\prime}, \sigma^{\prime \prime} \omega_{\mathbf{k}-\mathbf{k}^{\prime}}^{\gamma}\right)\right|^{2}}{\epsilon^{*}\left(\mathbf{k}, \omega^{\prime}+\sigma^{\prime \prime} \omega_{\mathbf{k}-\mathbf{k}^{\prime}}^{\gamma}\right.} I_{\mathbf{k}-\mathbf{k}^{\prime \prime}}^{\sigma^{\prime \prime} \gamma}\left(\omega-\omega^{\prime}-\sigma^{\prime \prime} \omega_{\mathbf{k}-\mathbf{k}^{\prime}}^{\gamma}\right)\right] \int d \mathbf{v} \delta\left(\omega^{\prime}-\mathbf{k}^{\prime} \cdot \mathbf{v}\right) f_{a}(\mathbf{p}) \\
& +2 \operatorname{Im} \sum_{a} \int d \mathbf{k}^{\prime} d \omega^{\prime} \frac{2\left(4 \pi e_{a}\right)^{2}}{(2 \pi)^{3}\left|\mathbf{k}-\mathbf{k}^{\prime}\right|^{2}\left|\epsilon\left(\mathbf{k}-\mathbf{k}^{\prime}, \omega-\omega^{\prime}\right)\right|^{2}} \\
& \times\left[\sum_{\sigma= \pm 1} \sum_{\alpha} \frac{\left\{\chi^{(2)}\left(\mathbf{k}^{\prime}, \omega^{\prime} \mid \mathbf{k}-\mathbf{k}^{\prime}, \sigma \omega_{\mathbf{k}}^{\alpha}-\omega^{\prime}\right)\right\}^{2}}{\epsilon\left(\mathbf{k}^{\prime}, \omega^{\prime}\right)} I_{\mathbf{k}}^{\sigma \alpha} \delta\left(\omega-\sigma \omega_{\mathbf{k}}^{\alpha}\right)\right. \\
& \left.-\sum_{\sigma^{\prime}= \pm 1} \sum_{\beta} \frac{\left|\chi^{(2)}\left(\mathbf{k}^{\prime}, \omega^{\prime} \mid \mathbf{k}-\mathbf{k}^{\prime}, \omega-\omega^{\prime}\right)\right|^{2}}{\epsilon^{*}(\mathbf{k}, \omega)} I_{\mathbf{k}^{\prime}}^{\sigma^{\prime} \beta} \delta\left(\omega^{\prime}-\sigma^{\prime} \omega_{\mathbf{k}^{\prime}}^{\beta}\right)\right] \int d \mathbf{p} \delta\left[\omega-\omega^{\prime}-\left(\mathbf{k}-\mathbf{k}^{\prime}\right) \cdot \mathbf{v}\right] f_{a}(\mathbf{p}) .
\end{aligned}
$$

Since the linear eigenmodes satisfy the dispersion relation, $\epsilon\left(\mathbf{k}, \sigma \omega_{\mathbf{k}}^{\alpha}\right) \approx 0, \alpha=L, S$, we have

$$
\frac{1}{\epsilon(\mathbf{k}, \omega)}=\mathcal{P} \frac{1}{\epsilon(\mathbf{k}, \omega)}-\sum_{\sigma= \pm 1} \sum_{\alpha=L, S} \frac{i \pi \delta\left(\omega-\sigma \omega_{\mathbf{k}}^{\alpha}\right)}{\epsilon^{\prime}\left(\mathbf{k}, \sigma \omega_{\mathbf{k}}^{\alpha}\right)}, \quad \frac{1}{\epsilon^{*}(\mathbf{k}, \omega)}=\mathcal{P} \frac{1}{\epsilon^{*}(\mathbf{k}, \omega)}+\sum_{\sigma= \pm 1} \sum_{\alpha=L, S} \frac{i \pi \delta\left(\omega-\sigma \omega_{\mathbf{k}}^{\alpha}\right)}{\epsilon^{\prime}\left(\mathbf{k}, \sigma \omega_{\mathbf{k}}^{\alpha}\right)},
$$

where a short-hand notation

$$
\epsilon^{\prime}(\mathbf{k}, \omega)=\frac{\partial \operatorname{Re} \epsilon(\mathbf{k}, \omega)}{\partial \omega}
$$

is used. The following relations hold for linear eigenmodes $\omega=\sigma \omega_{\mathbf{k}}^{\alpha}$, where $\alpha=L, S$ :

$$
\frac{1}{\epsilon^{\prime}\left(\mathbf{k}, \sigma \omega_{\mathbf{k}}^{L}\right)}=\frac{\sigma \omega_{\mathbf{k}}^{L}}{2}, \quad \frac{1}{\epsilon^{\prime}\left(\mathbf{k}, \sigma \omega_{\mathbf{k}}^{S}\right)}=\frac{\sigma \mu_{\mathbf{k}} \omega_{\mathbf{k}}^{L}}{2}, \quad \mu_{\mathbf{k}}=k^{3} \lambda_{D e}^{3} \sqrt{\frac{m_{e}}{m_{i}}}\left(1+\frac{3 T_{i}}{T_{e}}\right)^{1 / 2} .
$$

Equations (3.5) and (3.7) lead to the reduction of Eq. (3.4):

$$
\begin{aligned}
\frac{\partial I_{\mathbf{k}}^{\sigma \alpha}}{\partial t}= & -\frac{2 \operatorname{Im} \epsilon\left(\mathbf{k}, \sigma \omega_{\mathbf{k}}^{\alpha}\right)}{\epsilon^{\prime}\left(\mathbf{k}, \sigma \omega_{\mathbf{k}}^{\alpha}\right)} I_{\mathbf{k}}^{\sigma \alpha}+\sum_{a=e, i} \frac{4 e_{a}^{2}}{k^{2}\left[\epsilon^{\prime}\left(\mathbf{k}, \sigma \omega_{\mathbf{k}}^{\alpha}\right)\right]^{2}} \int d \mathbf{p} \delta\left(\sigma \omega_{\mathbf{k}}^{\alpha}-\mathbf{k} \cdot \mathbf{v}\right) f_{a}(\mathbf{p}) \\
& -\frac{4}{\epsilon^{\prime}\left(\mathbf{k}, \sigma \omega_{\mathbf{k}}^{\alpha}\right)} \sum_{\sigma^{\prime}= \pm 1} \sum_{\beta=L, S} \int d \mathbf{k}^{\prime} \operatorname{Im}\left[\mathcal{P} \frac{2\left\{\chi^{(2)}\left(\mathbf{k}^{\prime}, \sigma^{\prime} \omega_{\mathbf{k}^{\prime}}^{\beta} \mid \mathbf{k}-\mathbf{k}^{\prime}, \sigma \omega_{\mathbf{k}}^{\alpha}-\sigma^{\prime} \omega_{\mathbf{k}^{\prime}}^{\beta}\right)\right\}^{2}}{\epsilon\left(\mathbf{k}-\mathbf{k}^{\prime}, \sigma \omega_{\mathbf{k}}^{\alpha}-\sigma^{\prime} \omega_{\mathbf{k}^{\prime}}^{\beta}\right)}\right. \\
& \left.-\bar{\chi}^{(3)}\left(\mathbf{k}^{\prime}, \sigma^{\prime} \omega_{\mathbf{k}^{\prime}}^{\beta}\left|-\mathbf{k}^{\prime},-\sigma^{\prime} \omega_{\mathbf{k}^{\prime}}^{\beta}\right| \mathbf{k}, \sigma \omega_{\mathbf{k}}^{\alpha}\right)\right] I_{\mathbf{k}^{\prime}}^{\sigma^{\prime} \beta} I_{\mathbf{k}}^{\sigma \alpha} \\
& -\sum_{a=e, i} \frac{16 e_{a}^{2}}{\epsilon^{\prime}\left(\mathbf{k}, \sigma \omega_{\mathbf{k}}^{\alpha}\right)} \sum_{\sigma^{\prime}= \pm 1} \sum_{\beta=L, S} \int d \mathbf{k}^{\prime} \frac{\left|\chi^{(2)}\left(\mathbf{k}^{\prime}, \sigma^{\prime} \omega_{\mathbf{k}^{\prime}}^{\beta} \mid \mathbf{k}-\mathbf{k}^{\prime}, \sigma \omega_{\mathbf{k}}^{\alpha}-\sigma^{\prime} \omega_{\mathbf{k}^{\prime}}^{\beta}\right)\right|^{2}}{\left|\mathbf{k}-\mathbf{k}^{\prime}\right|^{2}\left|\epsilon\left(\mathbf{k}-\mathbf{k}^{\prime}, \sigma \omega_{\mathbf{k}}^{\alpha}-\sigma^{\prime} \omega_{\mathbf{k}^{\prime}}^{\beta}\right)\right|^{2}} \\
& \times\left[\frac{I_{\mathbf{k}}^{\sigma \alpha}}{\epsilon\left(\mathbf{k}^{\prime}, \sigma^{\prime} \omega_{\mathbf{k}^{\prime}}^{\beta}\right)}-\frac{I_{\mathbf{k}^{\prime}}^{\sigma^{\prime} \beta}}{\epsilon^{\prime}\left(\mathbf{k}, \sigma \omega_{\mathbf{k}}^{\alpha}\right)}\right] \int d \mathbf{p} \delta\left[\sigma \omega_{\mathbf{k}}^{\alpha}-\sigma^{\prime} \omega_{\mathbf{k}^{\prime}}^{\beta}-\left(\mathbf{k}-\mathbf{k}^{\prime}\right) \cdot \mathbf{v}\right] f_{a}(\mathbf{p})
\end{aligned}
$$




$$
\begin{aligned}
& -\frac{4 \pi}{\epsilon^{\prime}\left(\mathbf{k}, \sigma \omega_{\mathbf{k}}^{\alpha}\right)} \sum_{\sigma^{\prime}, \sigma^{\prime \prime}= \pm 1} \sum_{\beta, \gamma=L, S} \int d \mathbf{k}^{\prime}\left|\chi^{(2)}\left(\mathbf{k}^{\prime}, \sigma^{\prime} \omega_{\mathbf{k}^{\prime}}^{\beta} \mid \mathbf{k}-\mathbf{k}^{\prime}, \sigma^{\prime \prime} \omega_{\mathbf{k}-\mathbf{k}^{\prime}}^{\gamma}\right)\right|^{2} \\
& \times\left[\frac{I_{\mathbf{k}-\mathbf{k}^{\prime}}^{\sigma^{\prime \prime} \gamma} I_{\mathbf{k}}^{\sigma \alpha}}{\epsilon^{\prime}\left(\mathbf{k}^{\prime}, \sigma^{\prime} \omega_{\mathbf{k}^{\prime}}^{\beta}\right)}+\frac{I_{\mathbf{k}^{\prime}}^{\sigma^{\prime} \beta} I_{\mathbf{k}}^{\sigma \alpha}}{\epsilon^{\prime}\left(\mathbf{k}-\mathbf{k}^{\prime}, \sigma^{\prime \prime} \omega_{\mathbf{k}-\mathbf{k}^{\prime}}^{\gamma}\right)}-\frac{I_{\mathbf{k}^{\prime}}^{\sigma^{\prime} \beta} I_{\mathbf{k}-\mathbf{k}^{\prime}}^{\sigma^{\prime \prime} \gamma}}{\epsilon^{\prime}\left(\mathbf{k}, \sigma \omega_{\mathbf{k}}^{\alpha}\right)}\right] \delta\left(\sigma \omega_{\mathbf{k}}^{\alpha}-\sigma^{\prime} \omega_{\mathbf{k}^{\prime}}^{\beta}-\sigma^{\prime \prime} \omega_{\mathbf{k}-\mathbf{k}^{\prime}}^{\gamma}\right) .
\end{aligned}
$$

This is the formal wave kinetic equation for conventional weak turbulence theory, which involves only the linear eigenmodes, namely, $\alpha=L$ and $S$. In this formalism, contributions from noneigenmode portions of the electric field fluctuations $\left\langle E^{2}\right\rangle_{\mathbf{k}, \omega \neq \sigma \omega_{\mathbf{k}}}$ are ignored. We will investigate the effects of including noneigenmodes in the generalized weak turbulence theory later. For the moment, we note that the first term on the right-hand corresponds to the induced emission, and the second term depicts the spontaneous emission. The third term represents the induced scattering, the fourth term describes the spontaneous scattering, and the final term depicts the decay processes. Of the terms depicting the decay processes, the first two terms within the large brackets are responsible for the induced decay process, while the final term corresponds to the spontaneous decay. We next calculate specific forms of each term in the above wave kinetic equation.

\section{A. Induced and spontaneous emissions}

We make use of nonrelativistic definition for momentum-velocity relation, $\mathbf{p}=m_{a} \mathbf{v}$, henceforth. Let us introduce the velocity distribution function with the ambient density explicitly taken out of the definition:

$$
f_{a}(\mathbf{v})=\hat{n} F_{a}(\mathbf{v})
$$

The induced emissions terms are given by

$$
\begin{aligned}
\left.\frac{\partial I_{\mathbf{k}}^{\sigma L}}{\partial t}\right|_{\text {ind. emiss. }} & =-\frac{2 \operatorname{Im} \epsilon\left(\mathbf{k}, \sigma \omega_{\mathbf{k}}^{L}\right)}{\epsilon^{\prime}\left(\mathbf{k}, \sigma \omega_{\mathbf{k}}^{L}\right)} I_{\mathbf{k}}^{L}=\pi \sigma \omega_{\mathbf{k}}^{L} \frac{\omega_{p e}^{2}}{k^{2}} \int d \mathbf{v} \delta\left(\sigma \omega_{\mathbf{k}}^{L}-\mathbf{k} \cdot \mathbf{v}\right) \mathbf{k} \cdot \frac{\partial F_{e}}{\partial \mathbf{v}} I_{\mathbf{k}}^{\sigma L}, \\
\left.\frac{\partial I_{\mathbf{k}}^{\sigma S}}{\partial t}\right|_{\text {ind. emiss. }} & =-\frac{2 \operatorname{Im} \epsilon\left(\mathbf{k}, \sigma \omega_{\mathbf{k}}^{S}\right)}{\epsilon^{\prime}\left(\mathbf{k}, \sigma \omega_{\mathbf{k}}^{S}\right)} I_{\mathbf{k}}^{S}=\pi \mu_{\mathbf{k}} \sigma \omega_{\mathbf{k}}^{L} \frac{\omega_{p e}^{2}}{k^{2}} \int d \mathbf{v} \delta\left(\sigma \omega_{\mathbf{k}}^{S}-\mathbf{k} \cdot \mathbf{v}\right) \mathbf{k} \cdot \frac{\partial}{\partial \mathbf{v}}\left(F_{e}+\frac{m_{e}}{m_{i}} F_{i}\right) I_{\mathbf{k}}^{\sigma S},
\end{aligned}
$$

where we have made use the definitions for the linear dielectric response function (2.39).

Spontaneous emission terms are given by

$$
\begin{aligned}
\left.\frac{\partial I_{\mathbf{k}}^{\sigma L}}{\partial t}\right|_{\text {spont. emiss. }} & =\sum_{a} \frac{4 e_{a}^{2}}{k^{2}\left[\epsilon^{\prime}\left(\mathbf{k}, \sigma \omega_{\mathbf{k}}^{L}\right)\right]^{2}} \int d \mathbf{v} \delta\left(\sigma \omega_{\mathbf{k}}^{L}-\mathbf{k} \cdot \mathbf{v}\right) f_{a}(\mathbf{v})=\frac{\hat{n} e^{2} \omega_{p e}^{2}}{k^{2}} \int d \mathbf{v} \delta\left(\sigma \omega_{\mathbf{k}}^{L}-\mathbf{k} \cdot \mathbf{v}\right) F_{e}, \\
\left.\frac{\partial I_{\mathbf{k}}^{\sigma S}}{\partial t}\right|_{\text {spont. emiss. }} & =\sum_{a} \frac{4 e_{a}^{2}}{k^{2}\left[\epsilon^{\prime}\left(\mathbf{k}, \sigma \omega_{\mathbf{k}}^{S}\right)\right]^{2}} \int d \mathbf{v} \delta\left(\sigma \omega_{\mathbf{k}}^{S}-\mathbf{k} \cdot \mathbf{v}\right) f_{a}(\mathbf{v})=\frac{\mu_{\mathbf{k}}^{2} \hat{n} e^{2} \omega_{p e}^{2}}{k^{2}} \int d \mathbf{v} \delta\left(\sigma \omega_{\mathbf{k}}^{S}-\mathbf{k} \cdot \mathbf{v}\right)\left(F_{e}+F_{i}\right) .
\end{aligned}
$$

\section{B. Induced and spontaneous decay processes}

Reference [9] discusses the various approximate and limiting forms of the nonlinear susceptibilities. We also make use of results obtained by one of us (P.H.Y.) in his earlier publications [16,17] in subsequent simplifications of various nonlinear susceptibilities. The following limiting forms for the second-order susceptibility are useful:

$$
\begin{aligned}
\chi_{a}^{(2)}\left(0, \omega_{1} \mid 0, \omega_{2}\right)= & 0, \quad \chi_{a}^{(2)}\left(\mathbf{k}_{1}, 0 \mid \mathbf{k}_{2}, 0\right)=-\frac{i e_{a}}{T_{a}} \frac{\omega_{p a}^{2}}{k_{1} k_{2}\left|\mathbf{k}_{1}+\mathbf{k}_{2}\right|} \frac{1}{v_{t h}^{a 2}}, \\
\chi_{a}^{(2)}\left(\mathbf{k}_{1}, \omega_{1} \mid \mathbf{k}_{2}, \omega_{2}\right)= & \frac{-i}{2} \frac{e_{a}}{m_{a}} \frac{\omega_{p a}^{2}}{\omega_{1} \omega_{2}\left(\omega_{1}+\omega_{2}\right)} \frac{1}{k_{1} k_{2}\left|\mathbf{k}_{1}+\mathbf{k}_{2}\right|}\left[\frac{k_{1}^{2} \mathbf{k}_{2} \cdot\left(\mathbf{k}_{1}+\mathbf{k}_{2}\right)}{\omega_{1}}+\frac{k_{2}^{2} \mathbf{k}_{1} \cdot\left(\mathbf{k}_{1}+\mathbf{k}_{2}\right)}{\omega_{2}}\right. \\
& \left.+\frac{\left(\mathbf{k}_{1}+\mathbf{k}_{2}\right)^{2} \mathbf{k}_{1} \cdot \mathbf{k}_{2}}{\omega_{1}+\omega_{2}}\right] \quad\left(\text { for } \omega_{1} \gg k_{1} v_{t h}^{a}, \omega_{2} \gg k_{2} v_{t h}^{a}, \text { and } \omega_{1}+\omega_{2} \gg\left|\mathbf{k}_{1}+\mathbf{k}_{2}\right| v_{t h}^{a}\right), \\
\chi_{a}^{(2)}\left(\mathbf{k}^{\prime}, \omega^{\prime} \mid \mathbf{k}-\mathbf{k}^{\prime}, \omega-\omega^{\prime}\right)= & \frac{i}{2} \frac{e_{a}}{m_{a}} \frac{k^{\prime}}{\omega\left(\omega-\omega^{\prime}\right)} \frac{\mathbf{k} \cdot\left(\mathbf{k}-\mathbf{k}^{\prime}\right)}{k\left|\mathbf{k}-\mathbf{k}^{\prime}\right|} \chi_{a}\left(\mathbf{k}^{\prime}, \omega^{\prime}\right) \\
\approx & \frac{i}{2} \frac{e_{a} \frac{\omega_{p a}^{2}}{T_{a}} \frac{\mathbf{k} \cdot\left(\mathbf{k}-\mathbf{k}^{\prime}\right)}{\omega\left(\omega-\omega^{\prime}\right)}\left(\text { for } \omega \gg k v_{t h}^{a}, \omega-\omega^{\prime} \gg\left|\mathbf{k}-\mathbf{k}^{\prime}\right|\right.}{\left.k k^{\prime}\left|\mathbf{k}^{\prime}\right| v_{t h}^{a}, \text { and } \omega^{\prime} \ll k^{\prime} v_{t h}^{a}\right),}
\end{aligned}
$$




$$
\begin{aligned}
\chi_{a}^{(2)}\left(\mathbf{k}^{\prime}, \omega^{\prime} \mid \mathbf{k}-\mathbf{k}^{\prime}, \omega-\omega^{\prime}\right) & =\frac{i}{2} \frac{e_{a}}{m_{a}} \frac{\left|\mathbf{k}-\mathbf{k}^{\prime}\right|}{\omega \cdot \mathbf{k}^{\prime}} \frac{\mathbf{k}^{\prime}}{k k^{\prime}} \chi_{a}\left(\mathbf{k}-\mathbf{k}^{\prime}, \omega-\omega^{\prime}\right) \\
& \approx \frac{i}{2} \frac{e_{a}}{T_{a}} \frac{\omega_{p a}^{2}}{\omega \omega^{\prime}} \frac{\mathbf{k} \cdot \mathbf{k}^{\prime}}{k k^{\prime}\left|\mathbf{k}-\mathbf{k}^{\prime}\right|} \quad\left(\text { for } \omega \gg k v_{t h}^{a}, \omega^{\prime} \gg k^{\prime} v_{t h}^{a}, \text { and } \omega-\omega^{\prime} \ll\left|\mathbf{k}-\mathbf{k}^{\prime}\right| v_{t h}^{a}\right), \\
\chi_{a}^{(2)}\left(\mathbf{k}^{\prime}, \omega^{\prime} \mid \mathbf{k}-\mathbf{k}^{\prime}, \omega-\omega^{\prime}\right) & =\frac{i}{2} \frac{e_{a}}{m_{a}} \frac{k}{\omega^{\prime}\left(\omega-\omega^{\prime}\right)} \frac{\mathbf{k}^{\prime} \cdot\left(\mathbf{k}-\mathbf{k}^{\prime}\right)}{k^{\prime}\left|\mathbf{k}-\mathbf{k}^{\prime}\right|} \chi_{a}(\mathbf{k}, \omega) \\
& \approx \frac{i}{2} \frac{e_{a}}{T_{a}} \frac{\omega_{p a}^{2}}{\omega^{\prime}\left(\omega-\omega^{\prime}\right)} \frac{\mathbf{k}^{\prime} \cdot\left(\mathbf{k}-\mathbf{k}^{\prime}\right)}{k k^{\prime}\left|\mathbf{k}-\mathbf{k}^{\prime}\right|} \quad\left(\text { for } \omega^{\prime} \gg k^{\prime} v_{t h}^{a}, \omega-\omega^{\prime} \gg\left|\mathbf{k}-\mathbf{k}^{\prime}\right| v_{t h}^{a}, \text { and } \omega \ll k v_{t h}^{a}\right) .
\end{aligned}
$$

In the above $v_{t h}^{a}=\sqrt{2 T_{a} / m_{a}}$ is the thermal speed for particle species $a$. The following approximate properties of third-order nonlinear susceptibility will also be used:

$$
\begin{aligned}
\bar{\chi}_{a}^{(3)}\left(\mathbf{k}^{\prime}, 0\left|-\mathbf{k}^{\prime}, 0\right| \mathbf{k}, 0\right)= & -\frac{e_{a}^{2}}{T_{a}^{2}} \frac{\omega_{p a}^{2}}{k^{2} k^{\prime 2}} \frac{\mathbf{k} \cdot \mathbf{k}^{\prime}}{k^{2}} \frac{1}{v_{t h}^{a 2}}, \quad \bar{\chi}_{a}^{(3)}\left(0, \omega^{\prime}\left|0,-\omega^{\prime}\right| 0, \omega\right)=0, \\
\bar{\chi}_{a}^{(3)}\left(\mathbf{k}^{\prime}, \omega^{\prime}\left|-\mathbf{k}^{\prime},-\omega^{\prime}\right| \mathbf{k}, \omega\right)= & \frac{1}{2} \frac{e_{a}^{2}}{m_{a}^{2}} \frac{\omega_{p a}^{2} \omega^{3} \omega^{\prime}}{k^{2} \cdot \mathbf{k}^{\prime}}\left[\frac{2 k^{2} k^{\prime 2}}{\omega \omega^{\prime}}+\frac{k^{\prime 2}\left[\mathbf{k} \cdot\left(\mathbf{k}-\mathbf{k}^{\prime}\right)\right]}{\omega^{\prime}\left(\omega-\omega^{\prime}\right)}+\frac{8 k^{2}\left(\mathbf{k} \cdot \mathbf{k}^{\prime}\right)}{\omega^{2}}+\frac{2\left(\mathbf{k} \times \mathbf{k}^{\prime}\right)^{2}+3 k^{2}\left[\mathbf{k}^{\prime} \cdot\left(\mathbf{k}-\mathbf{k}^{\prime}\right)\right]}{\omega\left(\omega-\omega^{\prime}\right)}\right. \\
& \left.+\frac{\left(\mathbf{k}-\mathbf{k}^{\prime}\right)^{2}\left(\mathbf{k} \cdot \mathbf{k}^{\prime}\right)}{\left(\omega-\omega^{\prime}\right)^{2}}\right] \quad\left(\text { for } \omega \gg k v_{t h}^{a}, \omega-\omega^{\prime} \gg\left|\mathbf{k}-\mathbf{k}^{\prime}\right| v_{t h}^{a}, \text { and } \omega^{\prime} \gg k^{\prime} v_{t h}^{a}\right), \\
\bar{\chi}_{a}^{(3)}\left(\mathbf{k}^{\prime}, \omega^{\prime}\left|-\mathbf{k}^{\prime},-\omega^{\prime}\right| \mathbf{k}, \omega\right)= & -\frac{1}{2 \omega^{3}} \frac{e_{a}^{2}}{m_{a}^{2}} \frac{\mathbf{k} \cdot \mathbf{k}^{\prime}}{k^{2}}\left[\frac{2 k^{2}}{\omega}+\frac{\mathbf{k} \cdot\left(\mathbf{k}-\mathbf{k}^{\prime}\right)}{\omega-\omega^{\prime}}\right] \chi_{a}\left(\mathbf{k}^{\prime}, \omega^{\prime}\right) \\
= & \left.\frac{i}{\omega^{3}} \frac{e_{a}}{m_{a}} \frac{\left(\mathbf{k} \cdot \mathbf{k}^{\prime}\right)\left|\mathbf{k}-\mathbf{k}^{\prime}\right|}{k k^{\prime}\left[\mathbf{k} \cdot\left(\mathbf{k}-\mathbf{k}^{\prime}\right)\right]}\left\{2 k^{2}\left(\omega-\omega^{\prime}\right)+\left[\mathbf{k} \cdot\left(\mathbf{k}-\mathbf{k}^{\prime}\right)\right] \omega\right\} \chi_{a}^{(2)}\left(\mathbf{k}^{\prime}, \omega^{\prime} \mid \mathbf{k}-\mathbf{k}^{\prime}, \omega-\omega^{\prime}\right) \quad \text { (for arbitrary } \omega^{\prime}\right), \\
\bar{\chi}_{a}^{(3)}\left(\mathbf{k}^{\prime}, \omega^{\prime}\left|-\mathbf{k}^{\prime},-\omega^{\prime}\right| \mathbf{k}, \omega\right)= & -\frac{1}{2} \frac{e_{a}^{2}}{m_{a}^{2}} \frac{\left|\mathbf{k}-\mathbf{k}^{\prime}\right|^{2}}{\omega^{3} \omega^{\prime}} \frac{\left(\mathbf{k} \cdot \mathbf{k}^{\prime}\right)^{2}}{k^{2} k^{\prime 2}} \chi_{a}\left(\mathbf{k}-\mathbf{k}^{\prime}, \omega-\omega^{\prime}\right) \\
= & \frac{i e_{a}}{m_{a}} \frac{\mathbf{k} \cdot \mathbf{k}^{\prime}}{k k^{\prime}} \frac{\left|\mathbf{k}-\mathbf{k}^{\prime}\right|}{\omega^{2}} \chi_{a}^{(2)}\left(\mathbf{k}^{\prime}, \omega^{\prime} \mid \mathbf{k}-\mathbf{k}^{\prime}, \omega-\omega^{\prime}\right) \quad\left(\text { for arbitrary } \omega-\omega^{\prime}\right) .
\end{aligned}
$$

Making use of Eq. (3.12), the decay processes can be obtained, following Refs. [16,17]. We note that the second-order nonlinear susceptibility is largely determined by the electrons, since the electrons with low mass are much more mobile than the ions, thus responding to perturbed electric field much more rapidly. We also note that Langmuir waves are fast waves satisfying $\omega_{\mathbf{k}}^{L} \gg k v_{t h}^{e}$, while the ion-sound frequency satisfies the slow mode condition, $\omega_{\mathbf{k}}^{S} \ll k v_{t h}^{e}$. This means that $\omega_{\mathbf{k}}^{S}$ may be treated as an arbitrary frequency. Various approximate forms of the second-order susceptibilities in Eq. (3.12) are then applicable, leading to

$$
\begin{aligned}
& \left.\frac{\partial I_{\mathbf{k}}^{\sigma L}}{\partial t}\right|_{\text {decay }}=-\frac{8 \pi}{\epsilon^{\prime}\left(\mathbf{k}, \sigma \omega_{\mathbf{k}}^{L}\right)} \sum_{\sigma^{\prime}, \sigma^{\prime \prime}= \pm 1} \int d \mathbf{k}^{\prime} \mid \chi^{(2)}\left(\mathbf{k}^{\prime}, \sigma^{\prime} \omega_{\mathbf{k}^{\prime}}^{L}\left|\mathbf{k}-\mathbf{k}^{\prime}, \sigma^{\prime \prime} \omega_{\mathbf{k}-\mathbf{k}^{\prime}}^{S}\right|^{2}\right. \\
& \times\left[\frac{I_{\mathbf{k}-\mathbf{k}^{\prime}}^{\sigma^{\prime \prime}} \sigma_{\mathbf{k}}^{\sigma L}}{\epsilon^{\prime}\left(\mathbf{k}^{\prime}, \sigma^{\prime} \omega_{\mathbf{k}^{\prime}}^{L}\right)}+\frac{I_{\mathbf{k}^{\prime}}^{\sigma^{\prime} L} I_{\mathbf{k}}^{\sigma L}}{\epsilon^{\prime}\left(\mathbf{k}-\mathbf{k}^{\prime}, \sigma^{\prime \prime} \omega_{\mathbf{k}-\mathbf{k}^{\prime}}^{S}\right)}-\frac{I_{\mathbf{k}^{\prime}}^{\sigma^{\prime} L} I_{\mathbf{k}-\mathbf{k}^{\prime}}^{\sigma^{\prime \prime} S}}{\epsilon^{\prime}\left(\mathbf{k}, \sigma \omega_{\mathbf{k}}^{L}\right)}\right] \delta\left(\sigma \omega_{\mathbf{k}}^{L}-\sigma^{\prime} \omega_{\mathbf{k}^{\prime}}^{L}-\sigma^{\prime \prime} \omega_{\mathbf{k}-\mathbf{k}^{\prime}}^{S}\right) \\
& =\frac{\pi}{2} \frac{e^{2}}{T_{e}^{2}} \sum_{\sigma^{\prime}, \sigma^{\prime \prime}= \pm 1} \sigma \omega_{\mathbf{k}}^{L} \int d \mathbf{k}^{\prime} \frac{\left(\mathbf{k} \cdot \mathbf{k}^{\prime}\right)^{2}}{k^{2} k^{\prime 2}\left|\mathbf{k}-\mathbf{k}^{\prime}\right|^{2}} \delta\left(\sigma \omega_{\mathbf{k}}^{L}-\sigma^{\prime} \omega_{\mathbf{k}^{\prime}}^{L}-\sigma^{\prime \prime} \omega_{\mathbf{k}-\mathbf{k}^{\prime}}^{S}\right) \\
& \times\left(\sigma \omega_{\mathbf{k}}^{L} I_{\mathbf{k}^{\prime}}^{\sigma^{\prime} L} I_{\mathbf{k}-\mathbf{k}^{\prime}}^{\sigma^{\prime \prime} S}-\sigma^{\prime} \omega_{\mathbf{k}^{\prime}}^{L} I_{\mathbf{k}-\mathbf{k}^{\prime}}^{\sigma^{\prime \prime} S} I_{\mathbf{k}}^{\sigma L}-\sigma^{\prime \prime} \mu_{\mathbf{k}-\mathbf{k}^{\prime}} \omega_{\mathbf{k}-\mathbf{k}^{\prime}}^{L}{ }_{\mathbf{k}^{\prime}}^{\sigma^{\prime} L} I_{\mathbf{k}}^{\sigma L}\right), \\
& \left.\frac{\partial I_{\mathbf{k}}^{\sigma S}}{\partial t}\right|_{\text {decay }}=-\frac{4 \pi}{\epsilon^{\prime}\left(\mathbf{k}, \sigma \omega_{\mathbf{k}}^{S}\right)} \sum_{\sigma^{\prime}, \sigma^{\prime \prime}= \pm 1} \int d \mathbf{k}^{\prime}\left|\chi^{(2)}\left(\mathbf{k}^{\prime}, \sigma^{\prime} \omega_{\mathbf{k}^{\prime}}^{L} \mid \mathbf{k}-\mathbf{k}^{\prime}, \sigma^{\prime \prime} \omega_{\mathbf{k}-\mathbf{k}^{\prime}}^{L}\right)\right|^{2} \\
& \times\left[\frac{I_{\mathbf{k}-\mathbf{k}^{\prime}}^{\sigma^{\prime \prime}} \sigma_{\mathbf{k}}^{\sigma S}}{\epsilon^{\prime}\left(\mathbf{k}^{\prime}, \sigma^{\prime} \omega_{\mathbf{k}^{\prime}}^{L}\right)}+\frac{I_{\mathbf{k}^{\prime}}^{\sigma^{\prime} L} I_{\mathbf{k}}^{\sigma S}}{\epsilon^{\prime}\left(\mathbf{k}-\mathbf{k}^{\prime}, \sigma^{\prime \prime} \omega_{\mathbf{k}-\mathbf{k}^{\prime}}^{L}\right)}-\frac{I_{\mathbf{k}^{\prime}}^{\sigma^{\prime} L} I_{\mathbf{k}-\mathbf{k}^{\prime}}^{\sigma^{\prime \prime} L}}{\epsilon^{\prime}\left(\mathbf{k}, \sigma \omega_{\mathbf{k}}^{S}\right)}\right] \delta\left(\sigma \omega_{\mathbf{k}}^{S}-\sigma^{\prime} \omega_{\mathbf{k}^{\prime}}^{L}-\sigma^{\prime \prime} \omega_{\mathbf{k}-\mathbf{k}^{\prime}}^{L}\right) \\
& =\frac{\pi}{4} \frac{e^{2}}{T_{e}^{2}} \sum_{\sigma^{\prime}, \sigma^{\prime \prime}= \pm 1} \sigma \mu_{\mathbf{k}} \omega_{\mathbf{k}}^{L} \int d \mathbf{k}^{\prime} \frac{\left[\mathbf{k}^{\prime} \cdot\left(\mathbf{k}-\mathbf{k}^{\prime}\right)\right]^{2}}{k^{2} k^{\prime 2}\left|\mathbf{k}-\mathbf{k}^{\prime}\right|^{2}} \delta\left(\sigma \omega_{\mathbf{k}}^{S}-\sigma^{\prime} \omega_{\mathbf{k}^{\prime}}^{L}-\sigma^{\prime \prime} \omega_{\mathbf{k}-\mathbf{k}^{\prime}}^{L}\right) \\
& \times\left(\sigma \mu_{\mathbf{k}} \omega_{\mathbf{k}}^{L} I_{\mathbf{k}^{\prime}}^{\sigma^{\prime} L} I_{\mathbf{k}-\mathbf{k}^{\prime}}^{\sigma^{\prime \prime} L}-\sigma^{\prime} \omega_{\mathbf{k}^{\prime}}^{L} \sigma_{\mathbf{k}-\mathbf{k}^{\prime}}^{\sigma^{\prime \prime}} \mathbf{k}_{\mathbf{k}}^{\sigma S}-\sigma^{\prime \prime} \omega_{\mathbf{k}-\mathbf{k}^{\prime}}^{L} I_{\mathbf{k}^{\prime}}^{\sigma^{\prime} L} I_{\mathbf{k}}^{\sigma S}\right) .
\end{aligned}
$$


The first terms on the right-hand sides represent the spontaneous decay processes, while the remaining two terms correspond to induced decays.

\section{Induced and spontaneous scattering processes}

For this process, earlier Refs. [16,17] discussed the derivation in detail. For $L$ mode, Refs. [16,17] shows that the most important terms are induced scattering involving two $L$ modes. For the second-order nonlinear susceptibility of relevance to such a process, one may treat $\sigma \omega_{\mathbf{k}}^{L}-\sigma^{\prime} \omega_{\mathbf{k}^{\prime}}^{L}$ as the slow mode. One may also approximate the real part of the dielectric constant $\epsilon\left(\mathbf{k}-\mathbf{k}^{\prime}, \sigma \omega_{\mathbf{k}}^{L}-\sigma^{\prime} \omega_{\mathbf{k}^{\prime}}^{L}\right)$ by $1 /\left|\mathbf{k}-\mathbf{k}^{\prime}\right|^{2} \lambda_{D e}^{2}$. The imaginary part of the above dielectric constant has both electron and ion contributions. Upon treating the imaginary part as a small quantity, one may expand the term $1 / \epsilon\left(\mathbf{k}-\mathbf{k}^{\prime}, \sigma \omega_{\mathbf{k}}^{L}-\sigma^{\prime} \omega_{\mathbf{k}^{\prime}}^{L}\right)$ as $1 /\left|\mathbf{k}-\mathbf{k}^{\prime}\right|^{2} \lambda_{D e}^{2}-i \operatorname{Im} \epsilon\left(\mathbf{k}-\mathbf{k}^{\prime}, \sigma \omega_{\mathbf{k}}^{L}-\sigma^{\prime} \omega_{\mathbf{k}^{\prime}}^{L}\right)$. The third-order nonlinear susceptibility can also be approximated by treating $\sigma \omega_{\mathbf{k}}^{L}-\sigma^{\prime} \omega_{\mathbf{k}^{\prime}}^{L}$ as arbitrary (slow mode) frequency. The resulting expression, which was derived in the above references, is given by

$$
\begin{aligned}
\left.\frac{\partial I_{\mathbf{k}}^{\sigma L}}{\partial t}\right|_{\text {ind. sc. }}= & -\frac{4}{\epsilon^{\prime}\left(\mathbf{k}, \sigma \omega_{\mathbf{k}}^{\alpha}\right)} \sum_{\sigma^{\prime}= \pm 1} \int d \mathbf{k}^{\prime} \operatorname{Im}\left[\mathcal{P} \frac{2\left\{\chi^{(2)}\left(\mathbf{k}^{\prime}, \sigma^{\prime} \omega_{\mathbf{k}^{\prime}}^{L} \mid \mathbf{k}-\mathbf{k}^{\prime}, \sigma \omega_{\mathbf{k}}^{L}-\sigma^{\prime} \omega_{\mathbf{k}^{\prime}}^{L}\right)\right\}^{2}}{\epsilon\left(\mathbf{k}-\mathbf{k}^{\prime}, \sigma \omega_{\mathbf{k}}^{L}-\sigma^{\prime} \omega_{\mathbf{k}^{\prime}}^{L}\right)}\right. \\
& \left.-\bar{\chi}^{(3)}\left(\mathbf{k}^{\prime}, \sigma^{\prime} \omega_{\mathbf{k}^{\prime}}^{L}\left|-\mathbf{k}^{\prime},-\sigma^{\prime} \omega_{\mathbf{k}^{\prime}}^{L}\right| \mathbf{k}, \sigma \omega_{\mathbf{k}}^{L}\right)\right] I_{\mathbf{k}^{\prime}}^{\sigma^{\prime} L} I_{\mathbf{k}}^{\sigma L} \\
= & -\frac{\pi}{\omega_{p e}^{2}} \frac{e^{2}}{m_{e}^{2}} \sum_{\sigma^{\prime}= \pm 1} \int d \mathbf{k}^{\prime} \int d \mathbf{v} \frac{\left(\mathbf{k} \cdot \mathbf{k}^{\prime}\right)^{2}}{k^{2} k^{\prime 2}} \delta\left[\sigma \omega_{\mathbf{k}}^{L}-\sigma^{\prime} \omega_{\mathbf{k}^{\prime}}^{L}-\left(\mathbf{k}-\mathbf{k}^{\prime}\right) \cdot \mathbf{v}\right] \\
& \times\left(\mathbf{k}-\mathbf{k}^{\prime}\right) \cdot \frac{\partial}{\partial \mathbf{v}}\left[\left(\sigma \omega_{\mathbf{k}}^{L}-\sigma^{\prime} \omega_{\mathbf{k}^{\prime}}^{L}\right) F_{e}-\frac{m_{e}}{m_{i}}\left(\sigma \omega_{\mathbf{k}}^{L}\right) F_{i}\right] I_{\mathbf{k}^{\prime}}^{\sigma^{\prime} L} I_{\mathbf{k}}^{\sigma L} .
\end{aligned}
$$

For $S$ waves, Refs. [16,17] discuss that the most important terms are those related to two $S$ mode waves nonlinearly interacting with the ions. The basic derivation that can be found in the above references is similar to that of $L$ mode scattering problem, which will not be repeated here. Instead, we present the final result,

$$
\begin{aligned}
\left.\frac{\partial I_{\mathbf{k}}^{\sigma S}}{\partial t}\right|_{\text {ind. sc. }}= & -\frac{4}{\epsilon^{\prime}\left(\mathbf{k}, \sigma \omega_{\mathbf{k}}^{\alpha}\right)} \sum_{\sigma^{\prime}= \pm 1} \int d \mathbf{k}^{\prime} \operatorname{Im}\left[\mathcal{P} \frac{2\left\{\chi^{(2)}\left(\mathbf{k}^{\prime}, \sigma^{\prime} \omega_{\mathbf{k}^{\prime}}^{S} \mid \mathbf{k}-\mathbf{k}^{\prime}, \sigma \omega_{\mathbf{k}}^{S}-\sigma^{\prime} \omega_{\mathbf{k}^{\prime}}^{\beta}\right)\right\}^{2}}{\epsilon\left(\mathbf{k}-\mathbf{k}^{\prime}, \sigma \omega_{\mathbf{k}}^{S}-\sigma^{\prime} \omega_{\mathbf{k}^{\prime}}^{S}\right)}\right. \\
& \left.-\bar{\chi}^{(3)}\left(\mathbf{k}^{\prime}, \sigma^{\prime} \omega_{\mathbf{k}^{\prime}}^{S}\left|-\mathbf{k}^{\prime},-\sigma^{\prime} \omega_{\mathbf{k}^{\prime}}^{S}\right| \mathbf{k}, \sigma \omega_{\mathbf{k}}^{S}\right)\right] I_{\mathbf{k}^{\prime}}^{\sigma^{\prime} S} I_{\mathbf{k}}^{\sigma S} \\
= & \frac{\pi}{\omega_{p e}^{2}} \frac{e^{2}}{m_{e} m_{i}} \mu_{\mathbf{k}}\left(\sigma \omega_{\mathbf{k}}^{L}\right) \sum_{\sigma^{\prime}= \pm 1} \int d \mathbf{k}^{\prime} \int d \mathbf{v} \frac{\left(\mathbf{k} \cdot \mathbf{k}^{\prime}\right)^{2}}{k^{4} k^{\prime 4} \lambda_{D e}^{4}}\left(W_{\mathbf{k}, \mathbf{k}^{\prime}}+\sigma \sigma^{\prime} \frac{k^{\prime}}{k}\right) \\
& \times\left(\mathbf{k}-\mathbf{k}^{\prime}\right) \cdot \frac{\partial F_{i}}{\partial \mathbf{v}} \delta\left[\sigma \omega_{\mathbf{k}}^{S}-\sigma^{\prime} \omega_{\mathbf{k}^{\prime}}^{S}-\left(\mathbf{k}-\mathbf{k}^{\prime}\right) \cdot \mathbf{v}\right] I_{\mathbf{k}^{\prime}}^{\sigma^{\prime} S} I_{\mathbf{k}}^{\sigma S},
\end{aligned}
$$

where

$$
\begin{aligned}
W_{\mathbf{k}, \mathbf{k}^{\prime}}= & {\left[1+\frac{\left|\mathbf{k}-\mathbf{k}^{\prime}\right|^{2}}{\left(\sigma k-\sigma^{\prime} k^{\prime}\right)^{2}}\right]^{2} \frac{1}{\left|\mathbf{k}-\mathbf{k}^{\prime}\right|^{4} \lambda_{D e}^{4}\left|\epsilon\left(\mathbf{k}-\mathbf{k}^{\prime}, \sigma \omega_{\mathbf{k}}^{S}-\sigma^{\prime} \omega_{\mathbf{k}^{\prime}}^{S}\right)\right|^{2}}, } \\
\left|\epsilon\left(\mathbf{k}-\mathbf{k}^{\prime}, \sigma \omega_{\mathbf{k}}^{S}-\sigma^{\prime} \omega_{\mathbf{k}^{\prime}}^{S}\right)\right|^{2} \approx & {\left[1+\frac{2\left(\mathbf{k} \cdot \mathbf{k}^{\prime}-\sigma \sigma^{\prime} k k^{\prime}\right)}{\left|\mathbf{k}-\mathbf{k}^{\prime}\right|^{2}\left(k-\sigma \sigma^{\prime} k^{\prime}\right)^{2} \lambda_{D e}^{2}}\right]^{2}+\frac{\pi}{2} \frac{m_{e}}{m_{i}} \frac{\left(k-\sigma \sigma^{\prime} k^{\prime}\right)^{2}}{\left|\mathbf{k}-\mathbf{k}^{\prime}\right|^{6} \lambda_{D e}^{4}}\left\{\exp \left[-\frac{m_{e}}{2 m_{i}} \frac{\left(k-\sigma \sigma^{\prime} k^{\prime}\right)^{2}}{\left|\mathbf{k}-\mathbf{k}^{\prime}\right|^{2}}\right]\right.} \\
& \left.+\left(\frac{m_{i}}{m_{e}}\right)^{1 / 2}\left(\frac{T_{e}}{T_{i}}\right)^{3 / 2} \exp \left[-\frac{T_{e}}{2 T_{i}} \frac{\left(k-\sigma \sigma^{\prime} k^{\prime}\right)^{2}}{\left|\mathbf{k}-\mathbf{k}^{\prime}\right|^{2}}\right]\right\}^{2} .
\end{aligned}
$$

Spontaneous scattering terms are given by [17]

$$
\begin{aligned}
\left.\frac{\partial I_{\mathbf{k}}^{\sigma \alpha}}{\partial t}\right|_{\text {spont. scatt. }}= & -\sum_{a} \frac{16 e_{a}^{2}}{\epsilon^{\prime}\left(\mathbf{k}, \sigma \omega_{\mathbf{k}}^{\alpha}\right)} \sum_{\sigma^{\prime}= \pm 1} \sum_{\beta=L, S} \int d \mathbf{k}^{\prime} \frac{\left|\chi^{(2)}\left(\mathbf{k}^{\prime}, \sigma^{\prime} \omega_{\mathbf{k}^{\prime}}^{\beta} \mid \mathbf{k}-\mathbf{k}^{\prime}, \sigma \omega_{\mathbf{k}}^{\alpha}-\sigma^{\prime} \omega_{\mathbf{k}^{\prime}}^{\beta}\right)\right|^{2}}{\left|\mathbf{k}-\mathbf{k}^{\prime}\right|^{2}\left|\epsilon\left(\mathbf{k}-\mathbf{k}^{\prime}, \sigma \omega_{\mathbf{k}}^{\alpha}-\sigma^{\prime} \omega_{\mathbf{k}^{\prime}}^{\beta}\right)\right|^{2}} \\
& \times\left[\frac{I_{\mathbf{k}}^{\sigma \alpha}}{\epsilon\left(\mathbf{k}^{\prime}, \sigma^{\prime} \omega_{\mathbf{k}^{\prime}}^{\beta}\right)}-\frac{I_{\mathbf{k}^{\prime}}^{\sigma^{\prime} \beta}}{\epsilon^{\prime}\left(\mathbf{k}, \sigma \omega_{\mathbf{k}}^{\alpha}\right)}\right] \int d \mathbf{v} \delta\left[\sigma \omega_{\mathbf{k}}^{\alpha}-\sigma^{\prime} \omega_{\mathbf{k}^{\prime}}^{\beta}-\left(\mathbf{k}-\mathbf{k}^{\prime}\right) \cdot \mathbf{v}\right] f_{a}(\mathbf{v}) .
\end{aligned}
$$


For the Langmuir waves Ref. [17] obtains

$$
\left.\frac{\partial I_{\mathbf{k}}^{\sigma L}}{\partial t}\right|_{\text {spont. scatt. }}=-\left(\sigma \omega_{\mathbf{k}}^{L}\right) \frac{\hat{n} e^{4}}{T_{e}^{2}} \lambda_{D e}^{4} \sum_{\sigma^{\prime}= \pm 1} \int d \mathbf{k}^{\prime} \int d \mathbf{v} \frac{\left(\mathbf{k} \cdot \mathbf{k}^{\prime}\right)^{2}}{k^{2} k^{\prime 2}}\left(\sigma^{\prime} \omega_{\mathbf{k}^{\prime}}^{L} I_{\mathbf{k}}^{\sigma L}-\sigma \omega_{\mathbf{k}}^{L} I_{\mathbf{k}^{\prime}}^{\sigma^{\prime} L}\right) \delta\left[\sigma \omega_{\mathbf{k}}^{L}-\sigma^{\prime} \omega_{\mathbf{k}^{\prime}}^{L}-\left(\mathbf{k}-\mathbf{k}^{\prime}\right) \cdot \mathbf{v}\right]\left(F_{e}+F_{i}\right) .
$$

For the ion-sound modes, Ref. [17] also obtains

$$
\begin{aligned}
\left.\frac{\partial I_{\mathbf{k}}^{\sigma S}}{\partial t}\right|_{\text {spont. scatt. }}= & -\frac{\hat{n} e^{4}}{\omega_{m_{e}^{2} p e}^{4}} \mu_{\mathbf{k}}\left(\sigma \omega_{\mathbf{k}}^{L}\right) \sum_{\sigma^{\prime}= \pm 1} \int d \mathbf{k}^{\prime} \int d \mathbf{v} \frac{\left(\mathbf{k} \cdot \mathbf{k}^{\prime}\right)^{2}}{k^{4} k^{\prime} \lambda^{4}{ }_{D e}^{4}} \\
& \times W_{\mathbf{k}, \mathbf{k}^{\prime}}\left(\mu_{\mathbf{k}^{\prime}} \sigma^{\prime} \omega_{\mathbf{k}^{\prime}}^{L} I_{\mathbf{k}}^{\sigma S}-\mu_{\mathbf{k}} \sigma \omega_{\mathbf{k}}^{L} I_{\mathbf{k}^{\prime}}^{\sigma^{\prime} S}\right) \delta\left[\sigma \omega_{\mathbf{k}}^{S}-\sigma^{\prime} \omega_{\mathbf{k}^{\prime}}^{S}-\left(\mathbf{k}-\mathbf{k}^{\prime}\right) \cdot \mathbf{v}\right]\left(F_{e}+F_{i}\right) .
\end{aligned}
$$

\section{Langmuir and ion-sound wave kinetic equations}

Following the convention employed in Refs. [16,17], we renormalize the ion-sound wave intensity with the factor $\mu_{\mathbf{k}}$ explicitly absorbed into the definition of the wave intensity:

$$
I_{\mathbf{k}}^{\sigma S} \rightarrow \frac{I_{\mathbf{k}}^{\sigma S}}{\mu_{\mathbf{k}}}
$$

We then obtain the following wave kinetic equations for the Langmuir $(L)$ and ion-sound $(S)$ modes, which can be constructed by adding all the terms that we have derived, namely, the induced emission terms in Eq. (3.10), terms representing the spontaneous emission, Eq. (3.11), terms depicting (spontaneous and induced) decay processes (3.14), induced scattering (3.15) and (3.16), and spontaneous scattering (3.19) and (3.20). Adding all the terms lead to the wave kinetic equations for linear eigenmodes for which only the contributions from linear eigenmodes in the electric field fluctuations are taken into account; see Eq. (3.2). The result is

$$
\begin{aligned}
\frac{\partial I_{\mathbf{k}}^{\sigma L}}{\partial t}= & S_{\mathbf{k}}^{\sigma L}+2 \gamma_{\mathbf{k}}^{\sigma L} I_{\mathbf{k}}^{\sigma L}+2 \sum_{\sigma^{\prime}, \sigma^{\prime \prime}} \int d \mathbf{k}^{\prime} v_{\mathbf{k}, \mathbf{k}^{\prime}}^{L}\left(\sigma \omega_{\mathbf{k}}^{L} I_{\mathbf{k}^{\prime}}^{\sigma^{\prime} L} I_{\mathbf{k}-\mathbf{k}^{\prime}}^{\sigma^{\prime \prime} S}-\sigma^{\prime} \omega_{\mathbf{k}^{\prime}}^{L} I_{\mathbf{k}-\mathbf{k}^{\prime}}^{\sigma^{\prime \prime} S} I_{\mathbf{k}}^{\sigma L}-\sigma^{\prime \prime} \omega_{\mathbf{k}-\mathbf{k}^{\prime}}^{L} I_{\mathbf{k}^{\prime}}^{\sigma^{\prime} L} I_{\mathbf{k}}^{\sigma L}\right) \\
& -\sum_{\sigma^{\prime}} \int d \mathbf{k}^{\prime}\left[u_{\mathbf{k}, \mathbf{k}^{\prime}}^{L}\left(\sigma^{\prime} \omega_{\mathbf{k}^{\prime}}^{L} I_{\mathbf{k}}^{\sigma L}-\sigma \omega_{\mathbf{k}}^{L} I_{\mathbf{k}^{\prime}}^{\sigma^{\prime} L}\right)-w_{\mathbf{k}, \mathbf{k}^{\prime}}^{L} I_{\mathbf{k}^{\prime}}^{\sigma^{\prime} L} I_{\mathbf{k}}^{\sigma L}\right], \\
\frac{\partial I_{\mathbf{k}}^{\sigma S}}{\partial t}= & S_{\mathbf{k}}^{\sigma S}+2 \gamma_{\mathbf{k}}^{\sigma S} I_{\mathbf{k}}^{\sigma S}+\sum_{\sigma^{\prime}, \sigma^{\prime \prime}= \pm 1} \int d \mathbf{k}^{\prime} v_{\mathbf{k}, \mathbf{k}^{\prime}}^{S}\left(\sigma \omega_{\mathbf{k}}^{L} I_{\mathbf{k}^{\prime}}^{\sigma^{\prime} L} I_{\mathbf{k}-\mathbf{k}^{\prime}}^{\sigma^{\prime \prime} L}-\sigma^{\prime} \omega_{\mathbf{k}}^{L} I_{\mathbf{k}-\mathbf{k}^{\prime}}^{\sigma^{\prime \prime} L} I_{\mathbf{k}}^{\sigma S}-\sigma^{\prime \prime} \omega_{\mathbf{k}-\mathbf{k}^{\prime}}^{L} I_{\mathbf{k}^{\prime}}^{\sigma^{\prime} L} I_{\mathbf{k}}^{\sigma S}\right) \\
& -\sum_{\sigma^{\prime}} \int d \mathbf{k}^{\prime}\left[u_{\mathbf{k}, \mathbf{k}^{\prime}}^{S}\left(\sigma^{\prime} \omega_{\mathbf{k}^{\prime}}^{L} I_{\mathbf{k}}^{\sigma S}-\sigma \omega_{\mathbf{k}}^{L} I_{\mathbf{k}^{\prime}}^{\sigma^{\prime} S}\right)-w_{\mathbf{k}, \mathbf{k}^{\prime}}^{S} I_{\mathbf{k}^{\prime}}^{\sigma^{\prime} S} I_{\mathbf{k}}^{\sigma S}\right],
\end{aligned}
$$

where the spontaneous emission terms are defined by

$$
S_{\mathbf{k}}^{\sigma L}=\frac{\hat{n} e^{2} \omega_{p e}^{2}}{k^{2}} \int d \mathbf{v} \delta\left(\sigma \omega_{\mathbf{k}}^{L}-\mathbf{k} \cdot \mathbf{v}\right) F_{e}(\mathbf{v}), \quad S_{\mathbf{k}}^{\sigma S}=\mu_{\mathbf{k}} \frac{\hat{n} e^{2} \omega_{p e}^{2}}{k^{2}} \int d \mathbf{v} \delta\left(\sigma \omega_{\mathbf{k}}^{S}-\mathbf{k} \cdot \mathbf{v}\right)\left[F_{e}(\mathbf{v})+F_{i}(\mathbf{v})\right],
$$

the induced emission terms are associated with the collisionless (Landau) damping rates, defined by

$$
\begin{aligned}
& \gamma_{\mathbf{k}}^{\sigma L}=\sigma \omega_{\mathbf{k}}^{L} \frac{\pi \omega_{p e}^{2}}{2 k^{2}} \int d \mathbf{v}\left[\mathbf{k} \cdot \frac{\partial F_{e}(\mathbf{v})}{\partial \mathbf{v}}\right] \delta\left(\sigma \omega_{\mathbf{k}}^{L}-\mathbf{k} \cdot \mathbf{v}\right), \\
& \gamma_{\mathbf{k}}^{\sigma S}=\sigma \mu_{\mathbf{k}} \omega_{\mathbf{k}}^{L} \frac{\pi \omega_{p e}^{2}}{2 k^{2}} \int d \mathbf{v}\left(\mathbf{k} \cdot \frac{\partial}{\partial \mathbf{v}}\right)\left[F_{e}(\mathbf{v})+\frac{m_{e}}{m_{i}} F_{i}(\mathbf{v})\right] \delta\left(\sigma \omega_{\mathbf{k}}^{S}-\mathbf{k} \cdot \mathbf{v}\right),
\end{aligned}
$$

the decay coefficients are redefined by absorbing the three-wave resonance delta functions,

$$
\begin{gathered}
v_{\mathbf{k}, \mathbf{k}^{\prime}}^{L}=\sigma \omega_{\mathbf{k}}^{L} \frac{\pi e^{2}}{4 T_{e}^{2}} \frac{\mu_{\mathbf{k}-\mathbf{k}^{\prime}}\left(\mathbf{k} \cdot \mathbf{k}^{\prime}\right)^{2}}{k^{2} k^{\prime 2}\left|\mathbf{k}-\mathbf{k}^{\prime}\right|^{2}} \delta\left(\sigma \omega_{\mathbf{k}}^{L}-\sigma^{\prime} \omega_{\mathbf{k}^{\prime}}^{L}-\sigma^{\prime \prime} \omega_{\mathbf{k}-\mathbf{k}^{\prime}}^{S}\right), \\
v_{\mathbf{k}, \mathbf{k}^{\prime}}^{S}=\sigma \omega_{\mathbf{k}}^{L} \frac{\pi e^{2}}{4 T_{e}^{2}} \frac{\mu_{\mathbf{k}}\left[\mathbf{k}^{\prime} \cdot\left(\mathbf{k}-\mathbf{k}^{\prime}\right)\right]^{2}}{k^{2} k^{\prime 2}\left|\mathbf{k}-\mathbf{k}^{\prime}\right|^{2}} \delta\left(\sigma \omega_{\mathbf{k}}^{S}-\sigma^{\prime} \omega_{\mathbf{k}^{\prime}}^{L}-\sigma^{\prime \prime} \omega_{\mathbf{k}-\mathbf{k}^{\prime}}^{L}\right), \\
033203-16
\end{gathered}
$$


the spontaneous scattering terms are defined in terms of the coefficients,

$$
\begin{aligned}
& u_{\mathbf{k}, \mathbf{k}^{\prime}}^{L}=\sigma \omega_{\mathbf{k}}^{L} \frac{\hat{n} e^{4}}{m_{e}^{2} \omega_{p e}^{4}} \frac{\left(\mathbf{k} \cdot \mathbf{k}^{\prime}\right)^{2}}{k^{2} k^{\prime 2}} \int d \mathbf{v} \delta\left[\sigma \omega_{\mathbf{k}}^{L}-\sigma^{\prime} \omega_{\mathbf{k}^{\prime}}^{L}-\left(\mathbf{k}-\mathbf{k}^{\prime}\right) \cdot \mathbf{v}\right]\left[F_{e}(\mathbf{v})+F_{i}(\mathbf{v})\right], \\
& u_{\mathbf{k}, \mathbf{k}^{\prime}}^{S}=\mu_{\mathbf{k}} \mu_{\mathbf{k}^{\prime}} \sigma \omega_{\mathbf{k}}^{L} \frac{\hat{n} e^{4}}{m_{e}^{2} \omega_{p e}^{4}} \frac{\left(\mathbf{k} \cdot \mathbf{k}^{\prime}\right)^{2}}{k^{4} k^{\prime 4} \lambda_{D e}^{4}} W_{\mathbf{k}, \mathbf{k}^{\prime}} \int d \mathbf{v} \delta\left[\sigma \omega_{\mathbf{k}}^{S}-\sigma^{\prime} \omega_{\mathbf{k}^{\prime}}^{S}-\left(\mathbf{k}-\mathbf{k}^{\prime}\right) \cdot \mathbf{v}\right]\left[F_{e}(\mathbf{v})+F_{i}(\mathbf{v})\right],
\end{aligned}
$$

and finally, the induced scattering terms are defined in terms of the coefficients:

$$
\begin{aligned}
& w_{\mathbf{k}, \mathbf{k}^{\prime}}^{L}=\frac{\pi e^{2}}{m_{e}^{2} \omega_{p e}^{2}} \frac{\left(\mathbf{k} \cdot \mathbf{k}^{\prime}\right)^{2}}{k^{2} k^{\prime 2}} \int d \mathbf{v}\left[\left(\mathbf{k}-\mathbf{k}^{\prime}\right) \cdot \frac{\partial}{\partial \mathbf{v}}\right]\left[\frac{m_{e}}{m_{i}} \sigma \omega_{\mathbf{k}}^{L} F_{i}(\mathbf{v})-\left(\sigma \omega_{\mathbf{k}}^{L}-\sigma^{\prime} \omega_{\mathbf{k}^{\prime}}^{L}\right) F_{e}(\mathbf{v})\right] \delta\left[\sigma \omega_{\mathbf{k}}^{L}-\sigma^{\prime} \omega_{\mathbf{k}^{\prime}}^{L}-\left(\mathbf{k}-\mathbf{k}^{\prime}\right) \cdot \mathbf{v}\right], \\
& w_{\mathbf{k}, \mathbf{k}^{\prime}}^{S}=\mu_{\mathbf{k}} \mu_{\mathbf{k}^{\prime}} \sigma \omega_{\mathbf{k}}^{L} \frac{\pi e^{2}}{m_{e} m_{i} \omega_{p e}^{2}} \frac{\left(\mathbf{k} \cdot \mathbf{k}^{\prime}\right)^{2}}{k^{4} k^{\prime 4} \lambda_{D e}^{4}}\left(W_{\mathbf{k}, \mathbf{k}^{\prime}}+\sigma \sigma^{\prime} \frac{k^{\prime}}{k}\right) \int d \mathbf{v}\left[\left(\mathbf{k}-\mathbf{k}^{\prime}\right) \cdot \frac{\partial F_{i}(\mathbf{v})}{\partial \mathbf{v}}\right] \delta\left[\sigma \omega_{\mathbf{k}}^{S}-\sigma^{\prime} \omega_{\mathbf{k}^{\prime}}^{S}-\left(\mathbf{k}-\mathbf{k}^{\prime}\right) \cdot \mathbf{v}\right] .
\end{aligned}
$$

In the induced emission term the Landau damping rate can become positive (instability) when the distribution has a positive velocity gradient. The induced scattering is sometimes called the nonlinear Landau damping. For $L$ mode, the induced scattering or nonlinear Landau damping has two pieces, one associated with the ions, i.e., the term with $F_{i}(\mathbf{v})$, and the other associated with the electrons, $F_{e}(\mathbf{v})$. Of the two, the ion Landau damping, also called the scattering off thermal ions, is the dominant term, and the electron Landau damping can be ignored for all practical purposes. However, we retain the electron term for the sake of completeness. In Eq. (3.27) the coefficient $W_{\mathbf{k}, \mathbf{k}^{\prime}}$ is defined by Eq. (3.17).

\section{E. Electron and ion particle kinetic equations}

The particle kinetic equation in the conventional weak turbulence theory that takes only the linear eigenmode contributions into consideration was already derived in Ref. [17]:

$$
\begin{aligned}
\frac{\partial F_{e}(\mathbf{v})}{\partial t} & =\frac{\pi e^{2}}{m_{e}^{2}} \sum_{\sigma} \int d \mathbf{k}\left(\frac{\mathbf{k}}{k} \cdot \frac{\partial}{\partial \mathbf{v}}\right) \delta\left(\sigma \omega_{\mathbf{k}}^{L}-\mathbf{k} \cdot \mathbf{v}\right)\left[\sigma \omega_{\mathbf{k}}^{L} \frac{\pi m_{e}}{4 \pi^{3} k} F_{e}(\mathbf{v})+I_{\mathbf{k}}^{\sigma L}\left\{\frac{\mathbf{k}}{k} \cdot \frac{\partial F_{e}(\mathbf{v})}{\partial \mathbf{v}}\right\}\right], \\
\frac{\partial F_{i}(\mathbf{v})}{\partial t} & =\frac{\pi e^{2}}{m_{i}^{2}} \sum_{\sigma} \int d \mathbf{k}\left(\frac{\mathbf{k}}{k} \cdot \frac{\partial}{\partial \mathbf{v}}\right) \mu_{\mathbf{k}} \delta\left(\sigma \omega_{\mathbf{k}}^{S}-\mathbf{k} \cdot \mathbf{v}\right)\left[\sigma \omega_{\mathbf{k}}^{L} \frac{\pi m_{i}}{4 \pi^{3} k} F_{i}(\mathbf{v})+I_{\mathbf{k}}^{\sigma S}\left\{\frac{\mathbf{k}}{k} \cdot \frac{\partial F_{i}(\mathbf{v})}{\partial \mathbf{v}}\right\}\right] .
\end{aligned}
$$

\section{WEAK TURBULENCE THEORY INCLUDING COLLISIONS: EFFECTS OF NONEIGENMODES}

\section{A. Absence of noneigenmode contribution to quasilinear wave kinetic equation}

Consider Eq. (2.59) without nonlinear terms:

$$
\frac{i}{2} \frac{\partial \operatorname{Re} \epsilon(\mathbf{k}, \omega)}{\partial \omega} \frac{\partial\left\langle\delta E^{2}\right\rangle_{\mathbf{k}, \omega}}{\partial t}+\operatorname{Re} \epsilon(\mathbf{k}, \omega)\left\langle\delta E^{2}\right\rangle_{\mathbf{k}, \omega}+i \operatorname{Im} \epsilon(\mathbf{k}, \omega)\left\langle\delta E^{2}\right\rangle_{\mathbf{k}, \omega}=\frac{2}{\pi} \frac{1}{k^{2} \epsilon^{*}(\mathbf{k}, \omega)} \sum_{a} e_{a}^{2} \int d \mathbf{v} \delta(\omega-\mathbf{k} \cdot \mathbf{v}) f_{a}(\mathbf{v}) .
$$

We reiterate that the dielectric constant is given by [see Eq. (2.39)]

$$
\epsilon(\mathbf{k}, \omega)=\sum_{a} \frac{4 \pi e_{a}^{2}}{m_{a} k^{2}} \int d \mathbf{v} \frac{\mathbf{k} \cdot \partial f_{a} / \partial \mathbf{v}}{\omega-\mathbf{k} \cdot \mathbf{v}+i 0}
$$

Taking the real part of Eq. (4.1), we have

$$
\operatorname{Re} \epsilon(\mathbf{k}, \omega)\left[\left\langle\delta E^{2}\right\rangle_{\mathbf{k}, \omega}-\frac{2}{\pi} \frac{1}{k^{2}|\epsilon(\mathbf{k}, \omega)|^{2}} \sum_{a} e_{a}^{2} \int d \mathbf{v} \delta(\omega-\mathbf{k} \cdot \mathbf{v}) f_{a}(\mathbf{v})\right]=0 .
$$

Notice two regimes of the wave number-frequency space. If one is interested in the region of $(\mathbf{k}, \omega)$ space for which $|\epsilon(\mathbf{k}, \omega)|^{2} \neq 0$, then one may balance the left- and right-hand sides of the above equation by writing

$$
\left\langle\delta E^{2}\right\rangle_{\mathbf{k}, \omega}=\left\langle\delta E^{2}\right\rangle_{\mathbf{k}, \omega}^{0}
$$

where

$$
\left\langle\delta E^{2}\right\rangle_{\mathbf{k}, \omega}^{0} \equiv \frac{2}{\pi} \frac{1}{k^{2}|\epsilon(\mathbf{k}, \omega)|^{2}} \sum_{a} e_{a}^{2} \int d \mathbf{v} \delta(\omega-\mathbf{k} \cdot \mathbf{v}) f_{a}(\mathbf{v}) .
$$

The validity of the solution (4.4) depends on the denominator $|\epsilon(\mathbf{k}, \omega)|^{2}$ not being zero. For eigenmodes $\epsilon\left(\mathbf{k}, \omega_{\mathbf{k}}^{\alpha}\right)=0$, which means that the denominator remains nonzero only if $\omega$ does not satisfy the dispersion relation, $\omega \neq \omega_{\mathbf{k}}^{\alpha}$. Therefore, the electric field fluctuation $\left\langle\delta E^{2}\right\rangle_{\mathbf{k}, \omega}^{0}$ must represent the contribution from noneigenmodes. The customary weak turbulence theory does not 
take such a contribution into account. Instead, the traditional theory is concerned only with the eigenmode contributions, as we have already outlined in Sec. III. Equation (3.1) defines the dispersion relation for the eigenmodes, where one is concerned with the vicinity of the zeros of $\operatorname{Re} \epsilon(\mathbf{k}, \omega)$. In the generalized theory, we may treat both the eigenmode and noneigenmode fluctuations by defining a quantity

$$
\Psi_{\mathbf{k}, \omega} \equiv\left\langle\delta E^{2}\right\rangle_{\mathbf{k}, \omega}-\left\langle\delta E^{2}\right\rangle_{\mathbf{k}, \omega}^{0}
$$

Then the function $\Psi_{\mathbf{k}, \omega}$ becomes the total eigenfunction of Eq. (4.3):

$$
\operatorname{Re} \epsilon(\mathbf{k}, \omega) \Psi_{\mathbf{k}, \omega}=0 .
$$

Consequently, $\Psi_{\mathbf{k}, \omega}$ can be expressed as

$$
\Psi_{\mathbf{k}, \omega}=\sum_{\sigma= \pm 1} \sum_{\alpha=L, S} I_{\mathbf{k}}^{\sigma \alpha} \delta\left(\omega-\sigma \omega_{\mathbf{k}}^{\alpha}\right)
$$

where $\sigma \omega_{\mathbf{k}}^{\alpha}$ is the eigenvalue, which satisfies

$$
\operatorname{Re} \epsilon\left(\mathbf{k}, \sigma \omega_{\mathbf{k}}^{\alpha}\right)=0 .
$$

Thus, we encompass two situations, that is, the collective modes that satisfy the eigenmode condition and the noneigenmode contribution,

$$
\left\langle\delta E^{2}\right\rangle_{\mathbf{k}, \omega}=\left\langle\delta E^{2}\right\rangle_{\mathbf{k}, \omega}^{0}+\sum_{\sigma= \pm 1} \sum_{\alpha=L, S} I_{\mathbf{k}}^{\sigma \alpha} \delta\left(\omega-\sigma \omega_{\mathbf{k}}^{\alpha}\right)
$$

which directly generalizes Eq. (3.2).

Upon substituting Eq. (4.10) to the imaginary part of Eq. (4.1), we have

$$
\begin{aligned}
& \epsilon^{\prime}(\mathbf{k}, \omega) \frac{\partial\left\langle\delta E^{2}\right\rangle_{\mathbf{k}, \omega}^{0}}{\partial t}+2 \operatorname{Im} \epsilon(\mathbf{k}, \omega)\left\langle\delta E^{2}\right\rangle_{\mathbf{k}, \omega}^{0}+\sum_{\sigma= \pm 1} \sum_{\alpha}\left[\epsilon^{\prime}\left(\mathbf{k}, \sigma \omega_{\mathbf{k}}^{\alpha}\right) \frac{\partial I_{\mathbf{k}}^{\sigma \alpha}}{\partial t}+2 \operatorname{Im} \epsilon\left(\mathbf{k}, \sigma \omega_{\mathbf{k}}^{\alpha}\right) I_{\mathbf{k}}^{\sigma \alpha}\right] \delta\left(\omega-\sigma \omega_{\mathbf{k}}^{\alpha}\right) \\
& \quad=\operatorname{Im} \frac{4}{\pi k^{2} \epsilon^{*}(\mathbf{k}, \omega)} \sum_{a} e_{a}^{2} \int d \mathbf{v} \delta(\omega-\mathbf{k} \cdot \mathbf{v}) f_{a}(\mathbf{v}) .
\end{aligned}
$$

We next make use of Eq. (3.5) to write the denominator $\epsilon^{*}(\mathbf{k}, \omega)$ on the right-hand side as the sum of the principal part and the eigenmode contribution, and use as well Eq. (4.5):

$$
\begin{aligned}
& \epsilon^{\prime}(\mathbf{k}, \omega) \frac{\partial\left\langle\delta E^{2}\right\rangle_{\mathbf{k}, \omega}^{0}}{\partial t}+\operatorname{Im} \frac{4}{\pi k^{2} \epsilon^{*}(\mathbf{k}, \omega)} \sum_{a} e_{a}^{2} \int d \mathbf{v} \delta(\omega-\mathbf{k} \cdot \mathbf{v}) f_{a}(\mathbf{v}) \\
& \quad+\sum_{\sigma= \pm 1} \sum_{\alpha}\left[\epsilon^{\prime}\left(\mathbf{k}, \sigma \omega_{\mathbf{k}}^{\alpha}\right) \frac{\partial I_{\mathbf{k}}^{\sigma \alpha}}{\partial t}+2 \operatorname{Im} \epsilon\left(\mathbf{k}, \sigma \omega_{\mathbf{k}}^{\alpha}\right) I_{\mathbf{k}}^{\sigma \alpha}\right] \delta\left(\omega-\sigma \omega_{\mathbf{k}}^{\alpha}\right) \\
& \quad=\operatorname{Im} \mathcal{P} \frac{4}{\pi k^{2} \epsilon^{*}(\mathbf{k}, \omega)} \sum_{a} e_{a}^{2} \int d \mathbf{v} \delta(\omega-\mathbf{k} \cdot \mathbf{v}) f_{a}(\mathbf{v})+\sum_{\sigma= \pm 1} \sum_{\alpha} \frac{4 \delta\left(\omega-\sigma \omega_{\mathbf{k}}^{\alpha}\right)}{k^{2} \epsilon^{\prime}\left(\mathbf{k}, \sigma \omega_{\mathbf{k}}^{\alpha}\right)} \sum_{a} e_{a}^{2} \int d \mathbf{v} \delta\left(\sigma \omega_{\mathbf{k}}^{\alpha}-\mathbf{k} \cdot \mathbf{v}\right) f_{a}(\mathbf{v}) .
\end{aligned}
$$

Within the definition for $\left\langle\delta E^{2}\right\rangle_{\mathbf{k}, \omega}^{0}$ appears the dielectric constant $\epsilon(\mathbf{k}, \omega)$. Since the argument of $\epsilon(\mathbf{k}, \omega)$ excludes the eigenmodes, the second term on the left-hand side of Eq. (4.12) is implicitly taken with the principal value. This means that it exactly cancels out the first term on the right-hand side of Eq. (4.12). If we assume that $\partial\left\langle\delta E^{2}\right\rangle_{\mathbf{k}, \omega}^{0} / \partial t=0$, then we are left with

$$
\sum_{\sigma= \pm 1} \sum_{\alpha}\left[\epsilon^{\prime}\left(\mathbf{k}, \sigma \omega_{\mathbf{k}}^{\alpha}\right) \frac{\partial I_{\mathbf{k}}^{\sigma \alpha}}{\partial t}+2 \operatorname{Im} \epsilon\left(\mathbf{k}, \sigma \omega_{\mathbf{k}}^{\alpha}\right) I_{\mathbf{k}}^{\sigma \alpha}\right] \delta\left(\omega-\sigma \omega_{\mathbf{k}}^{\alpha}\right)=\sum_{\sigma= \pm 1} \sum_{\alpha} \frac{4 \delta\left(\omega-\sigma \omega_{\mathbf{k}}^{\alpha}\right)}{k^{2} \epsilon^{\prime}\left(\mathbf{k}, \sigma \omega_{\mathbf{k}}^{\alpha}\right)} \sum_{a} e_{a}^{2} \int d \mathbf{v} \delta\left(\sigma \omega_{\mathbf{k}}^{\alpha}-\mathbf{k} \cdot \mathbf{v}\right) f_{a}(\mathbf{v}) .
$$

If we remove the common factor, $\sum_{\sigma= \pm 1} \sum_{\alpha} \delta\left(\omega-\sigma \omega_{\mathbf{k}}^{\alpha}\right)$, then we obtain

$$
\frac{\partial I_{\mathbf{k}}^{\sigma \alpha}}{\partial t}=-\frac{2 \operatorname{Im} \epsilon\left(\mathbf{k}, \sigma \omega_{\mathbf{k}}^{\alpha}\right)}{\epsilon^{\prime}\left(\mathbf{k}, \sigma \omega_{\mathbf{k}}^{\alpha}\right)} I_{\mathbf{k}}^{\sigma \alpha}+\sum_{a} \frac{4 e_{a}^{2}}{k^{2}\left[\epsilon^{\prime}\left(\mathbf{k}, \sigma \omega_{\mathbf{k}}^{\alpha}\right)\right]^{2}} \int d \mathbf{v} \delta\left(\sigma \omega_{\mathbf{k}}^{\alpha}-\mathbf{k} \cdot \mathbf{v}\right) f_{a}(\mathbf{v}),
$$

which is none other than the first two terms on the right-hand side of the conventional weak turbulence wave kinetic equation (3.22) that are dictated by linear wave-particle resonance conditions, $\sigma \omega_{\mathbf{k}}^{\alpha}-\mathbf{k} \cdot \mathbf{v}=0$. This shows that in the (quasi-) linear theory, noneigenmodes do not affect the wave kinetic equation. 


\section{B. Particle kinetic equation including collision integral}

Unlike the (quasi-) linear wave kinetic equation, the influence of noneigenmode electric field fluctuations on the particles is important, and it leads to the collision integral. The formal particle kinetic equation is given by Eq. (2.62). Inserting the total wave electric field intensity [Eq. (4.10)] and making explicit use of the definition for $\left\langle\delta E^{2}\right\rangle_{\mathbf{k}, \omega}^{0}[$ Eq. (4.5)], we have

$$
\begin{aligned}
\frac{\partial f_{a}}{\partial t}= & \frac{\pi e_{a}^{2}}{m_{a}^{2}} \int d \mathbf{k} \int d \omega\left(\frac{\mathbf{k}}{k} \cdot \frac{\partial}{\partial \mathbf{v}}\right) \delta(\omega-\mathbf{k} \cdot \mathbf{v})\left[\operatorname{Im} \mathcal{P} \frac{m_{a}}{2 \pi^{3} k \epsilon^{*}(\mathbf{k}, \omega)} f_{a}+\frac{2}{\pi} \frac{1}{k^{2}|\epsilon(\mathbf{k}, \omega)|^{2}}\right. \\
& \left.\times \sum_{b} e_{b}^{2} \int d \mathbf{v}^{\prime} \delta\left(\omega-\mathbf{k} \cdot \mathbf{v}^{\prime}\right) f_{b}\left(\mathbf{v}^{\prime}\right)\left(\frac{\mathbf{k}}{k} \cdot \frac{\partial f_{a}}{\partial \mathbf{v}}\right)+\sum_{\sigma} \sum_{\alpha} \frac{\pi m_{a} \delta\left(\omega-\sigma \omega_{\mathbf{k}}^{\alpha}\right)}{2 \pi^{3} k \epsilon^{\prime}\left(\mathbf{k}, \sigma \omega_{\mathbf{k}}^{\alpha}\right)} f_{a}+\sum_{\sigma} \sum_{\alpha} I_{\mathbf{k}}^{\sigma \alpha} \delta\left(\omega-\sigma \omega_{\mathbf{k}}^{\alpha}\right)\left(\frac{\mathbf{k}}{k} \cdot \frac{\partial f_{a}}{\partial \mathbf{v}}\right)\right] .
\end{aligned}
$$

Upon carrying out the $\omega$ integration, and making use of the definition for $\operatorname{Im} \epsilon(\mathbf{k}, \omega)$, we readily obtain the generalized particle kinetic equation that includes both the eigenmode and noneigenmode contributions:

$$
\begin{aligned}
\frac{\partial F_{a}(\mathbf{v})}{\partial t}= & \sum_{b} \frac{2 \hat{n} e_{a}^{2} e_{b}^{2}}{m_{a}^{2}} \frac{\partial}{\partial v_{i}} \int d \mathbf{k} \int d \mathbf{v}^{\prime} \frac{k_{i} k_{j}}{k^{4}} \frac{\delta\left(\mathbf{k} \cdot \mathbf{v}-\mathbf{k} \cdot \mathbf{v}^{\prime}\right)}{|\epsilon(\mathbf{k}, \mathbf{k} \cdot \mathbf{v})|^{2}}\left(\frac{\partial}{\partial v_{j}}-\frac{m_{a}}{m_{b}} \frac{\partial}{\partial v_{j}^{\prime}}\right) F_{a}(\mathbf{v}) F_{b}\left(\mathbf{v}^{\prime}\right) \\
& +\frac{\pi e_{a}^{2}}{m_{a}^{2}} \sum_{\sigma} \sum_{\alpha} \int d \mathbf{k}\left(\frac{\mathbf{k}}{k} \cdot \frac{\partial}{\partial \mathbf{v}}\right) \delta\left(\sigma \omega_{\mathbf{k}}^{\alpha}-\mathbf{k} \cdot \mathbf{v}\right)\left[\frac{\pi m_{a}}{2 \pi^{3} k \epsilon^{\prime}\left(\mathbf{k}, \sigma \omega_{\mathbf{k}}^{\alpha}\right)} F_{a}(\mathbf{v})+I_{\mathbf{k}}^{\sigma \alpha}\left\{\frac{\mathbf{k}}{k} \cdot \frac{\partial F_{a}(\mathbf{v})}{\partial \mathbf{v}}\right\}\right] .
\end{aligned}
$$

In the above, the first term on the right-hand side comes from noneigenmodes, while the second term represents the contribution from plasma normal modes. In Eq. (4.16), we have expressed the final result in terms of $F_{a}$, where the velocity distribution is defined by taking the ambient density factor $\hat{n}$ out of the distribution function, $f_{a}=\hat{n} F_{a}$. Equation (4.16) is the generalized particle kinetic equation that includes the well-known Balescu-Lenard collision integral, which is the first term on the right-hand side of Eq. (4.16). In comparison, Eq. (3.28), which only contains the velocity drag (or friction) term owing to the spontaneously generated electrostatic fluctuations and the velocity space diffusion term, the generalized kinetic equation for the particles (4.16) contains both the collision integral, which is the first term on the right-hand side, as well as the terms representing collective processes, that is, the second term on the right-hand side of Eq. (4.16).

In Eq. (4.16), the linear response function with the angular frequency $\omega$ replaced by $\mathbf{k} \cdot \mathbf{v}$ appears in the denominator of the first term on the right-hand side. Since the most important contributions to the noneigenmode fluctuations come from particles with the highest concentration, i.e., near $v=0$, we may treat these various angular frequency arguments having the basic form $\mathbf{k} \cdot \mathbf{v}$ as close to zero. We thus approximate

$$
\epsilon(\mathbf{k}, \mathbf{k} \cdot \mathbf{v}) \approx \epsilon(\mathbf{k}, 0)=1+\sum_{a} \frac{2 \omega_{p a}^{2}}{k^{2} v_{T a}^{2}}=1+\frac{2 \omega_{p e}^{2}}{k^{2} v_{T e}^{2}}\left(1+\frac{T_{e}}{T_{i}}\right) .
$$

If we further approximate $\epsilon(\mathbf{k}, \mathbf{k} \cdot \mathbf{v}) \approx 1$, then we must assume that $2\left[\omega_{p e} /\left(k v_{T e}\right)\right]^{2} \ll 1$. This assumption implies that for noneigenmode fluctuations, the wave number range should be restricted to short wavelength regime, $k^{2} \lambda_{D e}^{2} \gg 1$. Such an approximation leads to the Landau collision integral. Since the Landau integral contains $k$ integration that diverges for $k \rightarrow 0$ and $k \rightarrow \infty$, one heuristically limits the lower and upper $k$ integration to $\lambda_{D e}^{-1}<k<T_{a}\left|e_{a} e_{b}\right|^{-1}$, which leads to the well-known Coulomb logarithm, which in the case of electrons is given by $\ln \Lambda \equiv \ln \left(\lambda_{D e} T_{e} / e^{2}\right)=\ln \left(4 \pi \hat{n} \lambda_{D e}^{3}\right)$. Note that $4 \pi \hat{n} \lambda_{D e}^{3}$ represents the number of electrons in a sphere with radius $\lambda_{D e}$. Since the plasma parameter $g=1 /\left(\hat{n} \lambda_{D e}^{3}\right)$ is supposed to be small in order for the ionized gas to qualify as plasma, it can be seen that the quantity $\Lambda$ must be large. In the present discussion, however, we do not make the approximation to the dielectric constant that leads to the Landau collision integral, but rather, we adopt the approximation (4.17). This leads to

$$
\begin{aligned}
\frac{\partial F_{a}(\mathbf{v})}{\partial t}= & \sum_{b} \frac{2 \hat{n} e_{a}^{2} e_{b}^{2}}{m_{a}^{2}} \frac{\partial}{\partial v_{i}} \int d \mathbf{k} \int d \mathbf{v}^{\prime} \frac{k_{i} k_{j} \lambda_{D e}^{4} \delta\left(\mathbf{k} \cdot \mathbf{v}-\mathbf{k} \cdot \mathbf{v}^{\prime}\right)}{\left|1+T_{e} / T_{i}+k^{2} \lambda_{D e}^{2}\right|^{2}}\left(\frac{\partial}{\partial v_{j}}-\frac{m_{a}}{m_{b}} \frac{\partial}{\partial v_{j}^{\prime}}\right) F_{a}(\mathbf{v}) F_{b}\left(\mathbf{v}^{\prime}\right) \\
& +\frac{\pi e_{a}^{2}}{m_{a}^{2}} \sum_{\sigma} \sum_{\alpha} \int d \mathbf{k}\left(\frac{\mathbf{k}}{k} \cdot \frac{\partial}{\partial \mathbf{v}}\right) \delta\left(\sigma \omega_{\mathbf{k}}^{\alpha}-\mathbf{k} \cdot \mathbf{v}\right)\left[\frac{\pi m_{a}}{2 \pi^{3} k \epsilon^{\prime}\left(\mathbf{k}, \sigma \omega_{\mathbf{k}}^{\alpha}\right)} F_{a}(\mathbf{v})+I_{\mathbf{k}}^{\sigma \alpha}\left\{\frac{\mathbf{k}}{k} \cdot \frac{\partial F_{a}(\mathbf{v})}{\partial \mathbf{v}}\right\}\right]
\end{aligned}
$$

Note that the collision integral (the first term on the right-hand side) is but a variation of the Balescu-Lenard collision integral, or a modified Landau collision integral, the discussions of which can be found in the standard literature. The effects of noneigenmode fluctuations on the particle kinetic equation is thus to add the collision integral to the standard Fokker-Planck type of particle kinetic equation given by Eq. (3.28). This is not entirely new, although the systematic exposition of the mutual relationship between the eigenmode versus noneigenmode contributions to each term within the particle kinetic equation (4.18) may not have been made clearly in the existing literature. The real novelty and significance of the noneigenmode fluctuations appear in the wave dynamics, which we turn to next. 


\section{Wave kinetic equation including collisional damping and electrostatic bremsstrahlung}

We now consider Eq. (2.59) again, which is reproduced below:

$$
\begin{aligned}
\frac{i}{2} & \frac{\partial \operatorname{Re} \epsilon(\mathbf{k}, \omega)}{\partial \omega} \frac{\partial\left\langle\delta E^{2}\right\rangle_{\mathbf{k}, \omega}}{\partial t}+\operatorname{Re} \epsilon(\mathbf{k}, \omega)\left\langle\delta E^{2}\right\rangle_{\mathbf{k}, \omega}+i \operatorname{Im} \epsilon(\mathbf{k}, \omega)\left\langle\delta E^{2}\right\rangle_{\mathbf{k}, \omega}-\frac{2}{\pi} \frac{1}{k^{2} \epsilon^{*}(\mathbf{k}, \omega)} \sum_{a} e_{a}^{2} \int d \mathbf{v} \delta(\omega-\mathbf{k} \cdot \mathbf{v}) f_{a}(\mathbf{v}) \\
= & -2 \int d \mathbf{k}^{\prime} \int d \omega^{\prime}\left[\left\{\chi^{(2)}\left(\mathbf{k}^{\prime}, \omega^{\prime} \mid \mathbf{k}-\mathbf{k}^{\prime}, \omega-\omega^{\prime}\right)\right\}^{2}\left\{\frac{\left\langle\delta E^{2}\right\rangle_{\mathbf{k}-\mathbf{k}^{\prime}, \omega-\omega^{\prime}}}{\epsilon\left(\mathbf{k}^{\prime}, \omega^{\prime}\right)}+\frac{\left\langle\delta E^{2}\right\rangle_{\mathbf{k}^{\prime}, \omega^{\prime}}}{\epsilon\left(\mathbf{k}-\mathbf{k}^{\prime}, \omega-\omega^{\prime}\right)}\right\}\right. \\
& \left.-\bar{\chi}^{(3)}\left(\mathbf{k}^{\prime}, \omega^{\prime}\left|-\mathbf{k}^{\prime},-\omega^{\prime}\right| \mathbf{k}, \omega\right)\left\langle\delta E^{2}\right\rangle_{\mathbf{k}^{\prime}, \omega^{\prime}}\right]\left\langle\delta E^{2}\right\rangle_{\mathbf{k}, \omega}+2 \int d \mathbf{k}^{\prime} \int d \omega^{\prime} \frac{\left|\chi^{(2)}\left(\mathbf{k}^{\prime}, \omega^{\prime} \mid \mathbf{k}-\mathbf{k}^{\prime}, \omega-\omega^{\prime}\right)\right|^{2}}{\epsilon^{*}(\mathbf{k}, \omega)}\left\langle\delta E^{2}\right\rangle_{\mathbf{k}^{\prime}, \omega^{\prime}}\left\langle\delta E^{2}\right\rangle_{\mathbf{k}-\mathbf{k}^{\prime}, \omega-\omega^{\prime}} \\
& -\frac{4}{\pi} \int d \mathbf{k}^{\prime} \int d \omega^{\prime} \frac{1}{k^{\prime 2}\left|\epsilon\left(\mathbf{k}^{\prime}, \omega^{\prime}\right)\right|^{2}}\left[\frac{\left\{\chi^{(2)}\left(\mathbf{k}^{\prime}, \omega^{\prime} \mid \mathbf{k}-\mathbf{k}^{\prime}, \omega-\omega^{\prime}\right)\right\}^{2}}{\epsilon\left(\mathbf{k}-\mathbf{k}^{\prime}, \omega-\omega^{\prime}\right)}\left\langle\delta E^{2}\right\rangle_{\mathbf{k}, \omega}\right. \\
& \left.-\frac{\left|\chi^{(2)}\left(\mathbf{k}^{\prime}, \omega^{\prime} \mid \mathbf{k}-\mathbf{k}^{\prime}, \omega-\omega^{\prime}\right)\right|^{2}}{\epsilon^{*}(\mathbf{k}, \omega)}\left\langle\delta E^{2}\right\rangle_{\mathbf{k}-\mathbf{k}^{\prime}, \omega-\omega^{\prime}}\right] \sum_{a} e_{a}^{2} \int d \mathbf{v} \delta\left(\omega^{\prime}-\mathbf{k}^{\prime} \cdot \mathbf{v}\right) f_{a}(\mathbf{v}) \\
& -\frac{4}{\pi} \int d \mathbf{k}^{\prime} \int d \omega^{\prime} \frac{1}{\left|\mathbf{k}-\mathbf{k}^{\prime}\right|^{2}\left|\epsilon\left(\mathbf{k}-\mathbf{k}^{\prime}, \omega-\omega^{\prime}\right)\right|^{2}}\left[\frac{\left\{\chi^{(2)}\left(\mathbf{k}^{\prime}, \omega^{\prime} \mid \mathbf{k}-\mathbf{k}^{\prime}, \omega-\omega^{\prime}\right)\right\}^{2}}{\epsilon\left(\mathbf{k}^{\prime}, \omega^{\prime}\right)}\left\langle\delta E^{2}\right\rangle_{\mathbf{k}, \omega}\right. \\
& \left.-\frac{\left|\chi^{(2)}\left(\mathbf{k}^{\prime}, \omega^{\prime} \mid \mathbf{k}-\mathbf{k}^{\prime}, \omega-\omega^{\prime}\right)\right|^{2}}{\epsilon^{*}(\mathbf{k}, \omega)}\left\langle\delta E^{2}\right\rangle_{\mathbf{k}^{\prime}, \omega^{\prime}}\right] \sum_{a} e_{a}^{2} \int d \mathbf{v} \delta\left[\omega-\omega^{\prime}-\left(\mathbf{k}-\mathbf{k}^{\prime}\right) \cdot \mathbf{v}\right] f_{a}(\mathbf{v})
\end{aligned}
$$

In the above, terms on the left-hand side correspond to linear processes, which we have already discussed. All the terms that appear to the right are characterized by nonlinear interactions. We substitute Eq. (4.10) for the total electric field fluctuation, $\left\langle\delta E^{2}\right\rangle_{\mathbf{k}, \omega}$, to the nonlinear terms. We already established that the linear terms are unaffected by such a procedure. Consequently we pay attention only to the nonlinear terms. Since we are interested in $\omega=\sigma \omega_{\mathbf{k}}^{\alpha}$ in the end, those terms containing $\left\langle\delta E^{2}\right\rangle_{\mathbf{k}, \omega}^{0}$ can be ignored at the outset, since such terms are by definition, excluded from the region satisfying $\omega=\sigma \omega_{\mathbf{k}}^{\alpha}$. However, noneigenmode fluctuations having different arguments, $\left\langle\delta E^{2}\right\rangle_{\mathbf{k}^{\prime}, \omega^{\prime}}^{0}$ and $\left\langle\delta E^{2}\right\rangle_{\mathbf{k}-\mathbf{k}^{\prime}, \omega-\omega^{\prime}}^{0}$, must be retained. This leads to the following equation that shows the balance of nonlinear terms on the right-hand side of Eq. (2.59):

$$
\begin{aligned}
& \text { NL terms }=-2 \int d \mathbf{k}^{\prime} \int d \omega^{\prime}\left\{\left\{\chi^{(2)}\left(\mathbf{k}^{\prime}, \omega^{\prime} \mid \mathbf{k}-\mathbf{k}^{\prime}, \omega-\omega^{\prime}\right)\right\}^{2}\right. \\
& \times\left[\frac{\overbrace{\left\langle\delta E^{2}\right\rangle_{\mathbf{k}-\mathbf{k}^{\prime}, \omega-\omega^{\prime}}^{0}}+\sum_{\sigma^{\prime \prime}} \sum_{\gamma} I_{\mathbf{k}-\mathbf{k}^{\prime}}^{\sigma^{\prime \prime} \gamma} \delta\left(\omega-\omega^{\prime}-\sigma^{\prime \prime} \omega_{\mathbf{k}-\mathbf{k}^{\prime}}^{\gamma}\right)}{\epsilon\left(\mathbf{k}^{\prime}, \omega^{\prime}\right)}+\frac{\overbrace{\left\langle\delta E^{2}\right\rangle_{\mathbf{k}^{\prime}, \omega^{\prime}}^{0}}+\sum_{\sigma^{\prime}} \sum_{\beta} I_{\mathbf{k}^{\prime}}^{\sigma^{\prime} \beta} \delta\left(\omega^{\prime}-\sigma^{\prime} \omega_{\mathbf{k}^{\prime}}^{\beta}\right)}{\epsilon\left(\mathbf{k}-\mathbf{k}^{\prime}, \omega-\omega^{\prime}\right)}\right] \\
& \left.-\bar{\chi}^{(3)}\left(\mathbf{k}^{\prime}, \omega^{\prime}\left|-\mathbf{k}^{\prime},-\omega^{\prime}\right| \mathbf{k}, \omega\right)[\overbrace{\left\langle\delta E^{2}\right\rangle_{\mathbf{k}^{\prime}, \omega^{\prime}}^{0}}+\sum_{\sigma^{\prime}} \sum_{\beta} I_{\mathbf{k}^{\prime}}^{\sigma^{\prime} \beta} \delta\left(\omega^{\prime}-\sigma^{\prime} \omega_{\mathbf{k}^{\prime}}^{\beta}\right)]\right\} \sum_{\sigma} \sum_{\alpha} I_{\mathbf{k}}^{\sigma \alpha} \delta\left(\omega-\sigma \omega_{\mathbf{k}}^{\alpha}\right) \\
& +2 \int d \mathbf{k}^{\prime} \int d \omega^{\prime} \frac{\left|\chi^{(2)}\left(\mathbf{k}^{\prime}, \omega^{\prime} \mid \mathbf{k}-\mathbf{k}^{\prime}, \omega-\omega^{\prime}\right)\right|^{2}}{\epsilon^{*}(\mathbf{k}, \omega)}[\overbrace{\left\langle\delta E^{2}\right\rangle_{\mathbf{k}^{\prime}, \omega^{\prime}}^{0}}+\sum_{\sigma^{\prime}} \sum_{\beta} I_{\mathbf{k}^{\prime}}^{\sigma^{\prime} \beta} \delta\left(\omega^{\prime}-\sigma^{\prime} \omega_{\mathbf{k}^{\prime}}^{\beta}\right)] \\
& \times[\overbrace{\left\langle\delta E^{2}\right\rangle_{\mathbf{k}-\mathbf{k}^{\prime}, \omega-\omega^{\prime}}^{0}}+\sum_{\sigma^{\prime \prime}} \sum_{\gamma} I_{\mathbf{k}-\mathbf{k}^{\prime}}^{\sigma^{\prime \prime} \gamma} \delta\left(\omega-\omega^{\prime}-\sigma^{\prime \prime} \omega_{\mathbf{k}-\mathbf{k}^{\prime}}^{\gamma}\right)] \\
& -\frac{4}{\pi} \int d \mathbf{k}^{\prime} \int d \omega^{\prime} \frac{1}{k^{\prime 2}\left|\epsilon\left(\mathbf{k}^{\prime}, \omega^{\prime}\right)\right|^{2}}\left\{\frac{\left\{\chi^{(2)}\left(\mathbf{k}^{\prime}, \omega^{\prime} \mid \mathbf{k}-\mathbf{k}^{\prime}, \omega-\omega^{\prime}\right)\right\}^{2}}{\epsilon\left(\mathbf{k}-\mathbf{k}^{\prime}, \omega-\omega^{\prime}\right)} \sum_{\sigma} \sum_{\alpha} I_{\mathbf{k}}^{\sigma \alpha} \delta\left(\omega-\sigma \omega_{\mathbf{k}}^{\alpha}\right)\right. \\
& \left.-\frac{\left|\chi^{(2)}\left(\mathbf{k}^{\prime}, \omega^{\prime} \mid \mathbf{k}-\mathbf{k}^{\prime}, \omega-\omega^{\prime}\right)\right|^{2}}{\epsilon^{*}(\mathbf{k}, \omega)}[\overbrace{\left\langle\delta E^{2}\right\rangle_{\mathbf{k}-\mathbf{k}^{\prime}, \omega-\omega^{\prime}}^{0}}+\sum_{\sigma^{\prime \prime}} \sum_{\gamma} I_{\mathbf{k}-\mathbf{k}^{\prime}}^{\sigma^{\prime \prime} \gamma} \delta\left(\omega-\omega^{\prime}-\sigma^{\prime \prime} \omega_{\mathbf{k}-\mathbf{k}^{\prime}}^{\gamma}\right)]\right\} \sum_{a} e_{a}^{2} \int d \mathbf{v} \delta\left(\omega^{\prime}-\mathbf{k}^{\prime} \cdot \mathbf{v}\right) f_{a}(\mathbf{v}) \\
& -\frac{4}{\pi} \int d \mathbf{k}^{\prime} \int d \omega^{\prime} \frac{1}{\left|\mathbf{k}-\mathbf{k}^{\prime}\right|^{2}\left|\epsilon\left(\mathbf{k}-\mathbf{k}^{\prime}, \omega-\omega^{\prime}\right)\right|^{2}}\left\{\frac{\left\{\chi^{(2)}\left(\mathbf{k}^{\prime}, \omega^{\prime} \mid \mathbf{k}-\mathbf{k}^{\prime}, \omega-\omega^{\prime}\right)\right\}^{2}}{\epsilon\left(\mathbf{k}^{\prime}, \omega^{\prime}\right)} \sum_{\sigma} \sum_{\alpha} I_{\mathbf{k}}^{\sigma \alpha} \delta\left(\omega-\sigma \omega_{\mathbf{k}}^{\alpha}\right)\right. \\
& \left.-\frac{\left|\chi^{(2)}\left(\mathbf{k}^{\prime}, \omega^{\prime} \mid \mathbf{k}-\mathbf{k}^{\prime}, \omega-\omega^{\prime}\right)\right|^{2}}{\epsilon^{*}(\mathbf{k}, \omega)}[\overbrace{\left\langle\delta E^{2}\right\rangle_{\mathbf{k}^{\prime}, \omega^{\prime}}^{0}}+\sum_{\sigma^{\prime}} \sum_{\beta} I_{\mathbf{k}^{\prime}}^{\sigma^{\prime} \beta} \delta\left(\omega^{\prime}-\sigma^{\prime} \omega_{\mathbf{k}^{\prime}}^{\beta}\right)]\right\} \sum_{a} e_{a}^{2} \int d \mathbf{v} \delta\left[\omega-\omega^{\prime}-\left(\mathbf{k}-\mathbf{k}^{\prime}\right) \cdot \mathbf{v}\right] f_{a}(\mathbf{v}) \text {. }
\end{aligned}
$$


In the above, we have indicated the new terms corresponding to noneigenmode fluctuations by overbraces $\sim$ in order to aid visual identification. Of the various terms above, if we are interested only in nonlinear terms that arise from collective excitations and spontaneous fluctuations in the eigenmode range, then we can ignore all source fluctuations associated with the superscript 0 , that is, the terms indicated by overbraces. In that case, one obtains the same results as Eqs. (3.8) or (3.22).

We now consider the correction terms coming from noneigenmode source fluctuations. Retaining only the correction terms, Eq. (4.19) is now written as

$$
\begin{aligned}
& \text { corr. }=-2 \int d \mathbf{k}^{\prime} \int d \omega^{\prime}\left\{\left\{\chi^{(2)}\left(\mathbf{k}^{\prime}, \omega^{\prime} \mid \mathbf{k}-\mathbf{k}^{\prime}, \omega-\omega^{\prime}\right)\right\}^{2}\left[\frac{\left\langle\delta E^{2}\right\rangle_{\mathbf{k}-\mathbf{k}^{\prime}, \omega-\omega^{\prime}}^{0}}{\epsilon\left(\mathbf{k}^{\prime}, \omega^{\prime}\right)}+\frac{\left\langle\delta E^{2}\right\rangle_{\mathbf{k}^{\prime}, \omega^{\prime}}^{0}}{\epsilon\left(\mathbf{k}-\mathbf{k}^{\prime}, \omega-\omega^{\prime}\right)}\right]\right. \\
& \left.-\bar{\chi}^{(3)}\left(\mathbf{k}^{\prime}, \omega^{\prime}\left|-\mathbf{k}^{\prime},-\omega^{\prime}\right| \mathbf{k}, \omega\right)\left\langle\delta E^{2}\right\rangle_{\mathbf{k}^{\prime}, \omega^{\prime}}^{0}\right\} \sum_{\sigma} \sum_{\alpha} I_{\mathbf{k}}^{\sigma \alpha} \delta\left(\omega-\sigma \omega_{\mathbf{k}}^{\alpha}\right) \\
& +2 \int d \mathbf{k}^{\prime} \int d \omega^{\prime} \frac{\left|\chi^{(2)}\left(\mathbf{k}^{\prime}, \omega^{\prime} \mid \mathbf{k}-\mathbf{k}^{\prime}, \omega-\omega^{\prime}\right)\right|^{2}}{\epsilon^{*}(\mathbf{k}, \omega)}\left[\left\langle\delta E^{2}\right\rangle_{\mathbf{k}^{\prime}, \omega^{\prime}}^{0}\left\langle\delta E^{2}\right\rangle_{\mathbf{k}-\mathbf{k}^{\prime}, \omega-\omega^{\prime}}^{0}\right. \\
& \left.+\left\langle\delta E^{2}\right\rangle_{\mathbf{k}-\mathbf{k}^{\prime}, \omega-\omega^{\prime}}^{0} \sum_{\sigma^{\prime}} \sum_{\beta} I_{\mathbf{k}^{\prime}}^{\sigma^{\prime} \beta} \delta\left(\omega^{\prime}-\sigma^{\prime} \omega_{\mathbf{k}^{\prime}}^{\beta}\right)+\left\langle\delta E^{2}\right\rangle_{\mathbf{k}^{\prime}, \omega^{\prime}}^{0} \sum_{\sigma^{\prime \prime}} \sum_{\gamma} I_{\mathbf{k}-\mathbf{k}^{\prime}}^{\sigma^{\prime \prime} \gamma} \delta\left(\omega-\omega^{\prime}-\sigma^{\prime \prime} \omega_{\mathbf{k}-\mathbf{k}^{\prime}}^{\gamma}\right)\right] \\
& +\frac{4}{\pi} \int d \mathbf{k}^{\prime} \int d \omega^{\prime} \frac{1}{k^{\prime 2}\left|\epsilon\left(\mathbf{k}^{\prime}, \omega^{\prime}\right)\right|^{2}} \frac{\left|\chi^{(2)}\left(\mathbf{k}^{\prime}, \omega^{\prime} \mid \mathbf{k}-\mathbf{k}^{\prime}, \omega-\omega^{\prime}\right)\right|^{2}}{\epsilon^{*}(\mathbf{k}, \omega)}\left\langle\delta E^{2}\right\rangle_{\mathbf{k}-\mathbf{k}^{\prime}, \omega-\omega^{\prime}}^{0} \sum_{a} e_{a}^{2} \int d \mathbf{v} \delta\left(\omega^{\prime}-\mathbf{k}^{\prime} \cdot \mathbf{v}\right) f_{a}(\mathbf{v}) \\
& +\frac{4}{\pi} \int d \mathbf{k}^{\prime} \int d \omega^{\prime} \frac{1}{\left|\mathbf{k}-\mathbf{k}^{\prime}\right|^{2}\left|\epsilon\left(\mathbf{k}-\mathbf{k}^{\prime}, \omega-\omega^{\prime}\right)\right|^{2}} \frac{\left|\chi^{(2)}\left(\mathbf{k}^{\prime}, \omega^{\prime} \mid \mathbf{k}-\mathbf{k}^{\prime}, \omega-\omega^{\prime}\right)\right|^{2}}{\epsilon^{*}(\mathbf{k}, \omega)}\left\langle\delta E^{2}\right\rangle_{\mathbf{k}^{\prime}, \omega^{\prime}}^{0} \\
& \times \sum_{a} e_{a}^{2} \int d \mathbf{v} \delta\left[\omega-\omega^{\prime}-\left(\mathbf{k}-\mathbf{k}^{\prime}\right) \cdot \mathbf{v}\right] f_{a}(\mathbf{v}) \text {. }
\end{aligned}
$$

Upon making use of the definition (4.5), namely, $\left\langle\delta E^{2}\right\rangle_{\mathbf{k}, \omega}^{0} \equiv\left\{2 /\left[\pi k^{2}|\epsilon(\mathbf{k}, \omega)|^{2}\right]\right\} \sum_{a} e_{a}^{2} \int d \mathbf{v} \delta(\omega-\mathbf{k} \cdot \mathbf{v}) f_{a}(\mathbf{v})$, we may rewrite Eq. (4.20) alternatively as

$$
\begin{aligned}
\text { corr. }= & -2 \int d \mathbf{k}^{\prime} \int d \omega^{\prime}\left[\left\{\chi^{(2)}\left(\mathbf{k}^{\prime}, \omega^{\prime} \mid \mathbf{k}-\mathbf{k}^{\prime}, \omega-\omega^{\prime}\right)\right\}^{2}\left[\frac{\left\langle\delta E^{2}\right\rangle_{\mathbf{k}-\mathbf{k}^{\prime}, \omega-\omega^{\prime}}^{0}}{\epsilon\left(\mathbf{k}^{\prime}, \omega^{\prime}\right)}+\frac{\left\langle\delta E^{2}\right\rangle_{\mathbf{k}^{\prime}, \omega^{\prime}}^{0}}{\epsilon\left(\mathbf{k}-\mathbf{k}^{\prime}, \omega-\omega^{\prime}\right)}\right]\right. \\
& \left.-\bar{\chi}^{(3)}\left(\mathbf{k}^{\prime}, \omega^{\prime}\left|-\mathbf{k}^{\prime},-\omega^{\prime}\right| \mathbf{k}, \omega\right)\left\langle\delta E^{2}\right\rangle_{\mathbf{k}^{\prime}, \omega^{\prime}}^{0}\right] \sum_{\sigma} \sum_{\alpha} I_{\mathbf{k}}^{\sigma \alpha} \delta\left(\omega-\sigma \omega_{\mathbf{k}}^{\alpha}\right) \\
& +2 \int d \mathbf{k}^{\prime} d \omega^{\prime} \frac{\left|\chi^{(2)}\left(\mathbf{k}^{\prime}, \omega^{\prime} \mid \mathbf{k}-\mathbf{k}^{\prime}, \omega-\omega^{\prime}\right)\right|^{2}}{\epsilon^{*}(\mathbf{k}, \omega)}\left[\left\langle\delta E^{2}\right\rangle_{\mathbf{k}-\mathbf{k}^{\prime}, \omega-\omega^{\prime}}^{0} \sum_{\sigma^{\prime}} \sum_{\beta} I_{\mathbf{k}^{\prime}}^{\sigma^{\prime} \beta} \delta\left(\omega^{\prime}-\sigma^{\prime} \omega_{\mathbf{k}^{\prime}}^{\beta}\right)\right. \\
& \left.+\left\langle\delta E^{2}\right\rangle_{\mathbf{k}^{\prime}, \omega^{\prime}}^{0} \sum_{\sigma^{\prime \prime}} \sum_{\gamma} I_{\mathbf{k}-\mathbf{k}^{\prime}}^{\sigma^{\prime \prime} \gamma} \delta\left(\omega-\omega^{\prime}-\sigma^{\prime \prime} \omega_{\mathbf{k}-\mathbf{k}^{\prime}}^{\gamma}\right)\right] \\
& +6 \int d \mathbf{k}^{\prime} \int d \omega^{\prime} \frac{\left|\chi^{(2)}\left(\mathbf{k}^{\prime}, \omega^{\prime} \mid \mathbf{k}-\mathbf{k}^{\prime}, \omega-\omega^{\prime}\right)\right|^{2}}{\epsilon^{*}(\mathbf{k}, \omega)}\left\langle\delta E^{2}\right\rangle_{\mathbf{k}^{\prime}, \omega^{\prime}}^{0}\left\langle\delta E^{2}\right\rangle_{\mathbf{k}-\mathbf{k}^{\prime}, \omega-\omega^{\prime}}^{0}
\end{aligned}
$$

Rewriting the noneigenmode fluctuations $\left\langle\delta E^{2}\right\rangle_{\mathbf{k}^{\prime}, \omega^{\prime}}^{0}$ and $\left\langle\delta E^{2}\right\rangle_{\mathbf{k}-\mathbf{k}^{\prime}, \omega-\omega^{\prime}}^{0}$ explicitly by making use of Eq. (4.5) we have

$$
\begin{aligned}
\text { corr. }= & -\sum_{a} \frac{4 e_{a}^{2}}{\pi} \sum_{\sigma} \sum_{\alpha} \int d \mathbf{k}^{\prime} \int d \mathbf{v}\left(\frac{2\left\{\chi ^ { ( 2 ) } \left(\mathbf{k}^{\prime}, \mathbf{k}^{\prime} \cdot \mathbf{v} \mid \mathbf{k}-\mathbf{k}^{\prime}, \sigma \omega_{\mathbf{k}}^{\alpha}-\mathbf{\mathbf { k } ^ { \prime } \cdot \mathbf { v } ) \} ^ { 2 }}\right.\right.}{\epsilon\left(\mathbf{k}-\mathbf{k}^{\prime}, \sigma \omega_{\mathbf{k}}^{\alpha}-\mathbf{k}^{\prime} \cdot \mathbf{v}\right)}\right. \\
& \left.-\bar{\chi}^{(3)}\left(\mathbf{k}^{\prime}, \mathbf{k}^{\prime} \cdot \mathbf{v}\left|-\mathbf{k}^{\prime},-\mathbf{k}^{\prime} \cdot \mathbf{v}\right| \mathbf{k}, \sigma \omega_{\mathbf{k}}^{\alpha}\right)\right) \frac{I_{\mathbf{k}}^{\sigma \alpha} f_{a}(\mathbf{v}) \delta\left(\omega-\sigma \omega_{\mathbf{k}}^{\alpha}\right)}{k^{\prime 2}\left|\epsilon\left(\mathbf{k}^{\prime}, \mathbf{k}^{\prime} \cdot \mathbf{v}\right)\right|^{2}} \\
& +\sum_{a} \frac{8 e_{a}^{2}}{\pi} \sum_{\sigma^{\prime}} \sum_{\beta} \int d \mathbf{k}^{\prime} \int d \mathbf{v} \frac{\left|\chi^{(2)}\left(\mathbf{k}^{\prime}, \sigma^{\prime} \omega_{\mathbf{k}^{\prime}}^{\beta} \mid \mathbf{k}-\mathbf{k}^{\prime}, \omega-\sigma^{\prime} \omega_{\mathbf{k}^{\prime}}^{\beta}\right)\right|^{2}}{\left|\mathbf{k}-\mathbf{k}^{\prime}\right|^{2} \epsilon^{*}(\mathbf{k}, \omega)\left|\epsilon\left(\mathbf{k}-\mathbf{k}^{\prime}, \omega-\sigma^{\prime} \omega_{\mathbf{k}^{\prime}}^{\beta}\right)\right|^{2}} I_{\mathbf{k}^{\prime}}^{\sigma^{\prime} \beta} f_{a}(\mathbf{v}) \delta\left[\omega-\sigma^{\prime} \omega_{\mathbf{k}^{\prime}}^{\beta}-\left(\mathbf{k}-\mathbf{k}^{\prime}\right) \cdot \mathbf{v}\right] \\
& +\sum_{a} \sum_{b} \frac{24 e_{a}^{2} e_{b}^{2}}{\pi^{2}} \int d \mathbf{k}^{\prime} \int d \mathbf{v} \int d \mathbf{v}^{\prime} \frac{\left|\chi^{(2)}\left[\mathbf{k}^{\prime}, \mathbf{k}^{\prime} \cdot \mathbf{v} \mid \mathbf{k}-\mathbf{k}^{\prime},\left(\mathbf{k}-\mathbf{k}^{\prime}\right) \cdot \mathbf{v}^{\prime}\right]\right|^{2}}{k^{\prime 2}\left|\mathbf{k}-\mathbf{k}^{\prime}\right|^{2} \epsilon^{*}(\mathbf{k}, \omega)\left|\epsilon\left[\mathbf{k}^{\prime}, \mathbf{k}^{\prime} \cdot \mathbf{v}\right)\right|^{2}\left|\epsilon\left(\mathbf{k}-\mathbf{k}^{\prime},\left(\mathbf{k}-\mathbf{k}^{\prime}\right) \cdot \mathbf{v}^{\prime}\right]\right|^{2}} \\
& \times \delta\left[\omega-\mathbf{k}^{\prime} \cdot \mathbf{v}-\left(\mathbf{k}-\mathbf{k}^{\prime}\right) \cdot \mathbf{v}^{\prime}\right] f_{a}(\mathbf{v}) f_{b}\left(\mathbf{v}^{\prime}\right),
\end{aligned}
$$


where we have carried out the $\omega^{\prime}$ integrations by virtue of the various delta functions, and rearranged certain terms by making use of the symmetry properties associated with the second-order nonlinear susceptibility.

Next, we make use of Eq. (3.5) to decompose the denominators $1 / \epsilon\left(\mathbf{k}-\mathbf{k}^{\prime}, \sigma \omega_{\mathbf{k}}^{\alpha}-\mathbf{k}^{\prime} \cdot \mathbf{v}\right)$ and $1 / \epsilon^{*}(\mathbf{k}, \omega)$ into principal parts and imaginary terms. Upon taking the imaginary part of the resulting equation, the nonlinear correction term that owes its existence to noneigenmode fluctuations finally emerges:

$$
\begin{aligned}
\text { corr. }= & -\sum_{a} \frac{4 e_{a}^{2}}{\pi} \int d \mathbf{k}^{\prime} \int d \mathbf{v} \frac{1}{k^{\prime 2}\left|\epsilon\left(\mathbf{k}^{\prime}, \mathbf{k}^{\prime} \cdot \mathbf{v}\right)\right|^{2}} \operatorname{Im}\left[\mathcal{P} \frac{2\left\{\chi^{(2)}\left(\mathbf{k}^{\prime}, \mathbf{k}^{\prime} \cdot \mathbf{v} \mid \mathbf{k}-\mathbf{k}^{\prime}, \sigma \omega_{\mathbf{k}}^{\alpha}-\mathbf{k}^{\prime} \cdot \mathbf{v}\right)\right\}^{2}}{\epsilon\left(\mathbf{k}-\mathbf{k}^{\prime}, \sigma \omega_{\mathbf{k}}^{\alpha}-\mathbf{k}^{\prime} \cdot \mathbf{v}\right)}\right. \\
& \left.-\bar{\chi}^{(3)}\left(\mathbf{k}^{\prime}, \mathbf{k}^{\prime} \cdot \mathbf{v}\left|-\mathbf{k}^{\prime},-\mathbf{k}^{\prime} \cdot \mathbf{v}\right| \mathbf{k}, \sigma \omega_{\mathbf{k}}^{\alpha}\right)\right] I_{\mathbf{k}}^{\sigma \alpha} f_{a}(\mathbf{v})-\sum_{\sigma^{\prime}} \sum_{\beta} \sum_{a} 8 e_{a}^{2} \int d \mathbf{k}^{\prime} \int d \mathbf{v} \frac{\left|\chi^{(2)}\left[\mathbf{k}^{\prime}, \sigma^{\prime} \omega_{\mathbf{k}^{\prime}}^{\beta} \mid \mathbf{k}-\mathbf{k}^{\prime},\left(\mathbf{k}-\mathbf{k}^{\prime}\right) \cdot \mathbf{v}\right]\right|^{2}}{\left|\mathbf{k}-\mathbf{k}^{\prime}\right|^{2}\left|\epsilon\left[\mathbf{k}-\mathbf{k}^{\prime},\left(\mathbf{k}-\mathbf{k}^{\prime}\right) \cdot \mathbf{v}\right]\right|^{2}} \\
& \times\left[\frac{I_{\mathbf{k}}^{\sigma \alpha}}{\epsilon^{\prime}\left(\mathbf{k}^{\prime}, \sigma^{\prime} \omega_{\mathbf{k}^{\prime}}^{\beta}\right)}-\frac{I_{\mathbf{k}^{\prime}}^{\sigma^{\prime} \beta}}{\epsilon^{\prime}\left(\mathbf{k}, \sigma \omega_{\mathbf{k}}^{\alpha}\right)}\right] \delta\left[\sigma \omega_{\mathbf{k}}^{\alpha}-\sigma^{\prime} \omega_{\mathbf{k}^{\prime}}^{\beta}-(\mathbf{k}-\mathbf{k}) \cdot \mathbf{v}\right] f_{a}(\mathbf{v}) \\
& +\sum_{a, b} \frac{24 e_{a}^{2} e_{b}^{2}}{\pi} \int d \mathbf{k}^{\prime} \int d \mathbf{v} \int d \mathbf{v}^{\prime} \frac{\left|\chi^{(2)}\left[\mathbf{k}^{\prime}, \mathbf{k}^{\prime} \cdot \mathbf{v}^{\prime} \mid \mathbf{k}-\mathbf{k}^{\prime},\left(\mathbf{k}-\mathbf{k}^{\prime}\right) \cdot \mathbf{v}\right]\right|^{2}}{k^{\prime 2}\left|\mathbf{k}-\mathbf{k}^{\prime}\right|^{2}\left|\epsilon\left(\mathbf{k}^{\prime}, \mathbf{k}^{\prime} \cdot \mathbf{v}^{\prime}\right)\right|^{2}\left|\epsilon\left[\mathbf{k}-\mathbf{k}^{\prime},\left(\mathbf{k}-\mathbf{k}^{\prime}\right) \cdot \mathbf{v}\right]\right|^{2}} \\
& \times \frac{\delta\left[\sigma \omega_{\mathbf{k}}^{\alpha}-\mathbf{k} \cdot \mathbf{v}+\mathbf{k}^{\prime} \cdot\left(\mathbf{v}-\mathbf{v}^{\prime}\right)\right]}{\epsilon^{\prime}\left(\mathbf{k}, \sigma \omega_{\mathbf{k}}^{\alpha}\right)} f_{a}(\mathbf{v}) f_{b}\left(\mathbf{v}^{\prime}\right) .
\end{aligned}
$$

The right-hand side of Eq. (4.23) is to be added to the right-hand side of Eq. (3.8). The result is a generalized wave kinetic equation that includes both the eigenmode and noneigenmode fluctuations, and which directly generalizes Eq. (3.8):

$$
\begin{aligned}
& \frac{\partial I_{\mathbf{k}}^{\sigma \alpha}}{\partial t}=-\frac{2 \operatorname{Im} \epsilon\left(\mathbf{k}, \sigma \omega_{\mathbf{k}}^{\alpha}\right)}{\epsilon^{\prime}\left(\mathbf{k}, \sigma \omega_{\mathbf{k}}^{\alpha}\right)} I_{\mathbf{k}}^{\sigma \alpha}+\sum_{a} \frac{4 e_{a}^{2}}{k^{2}\left[\epsilon^{\prime}\left(\mathbf{k}, \sigma \omega_{\mathbf{k}}^{\alpha}\right)\right]^{2}} \int d \mathbf{v} \delta\left(\sigma \omega_{\mathbf{k}}^{\alpha}-\mathbf{k} \cdot \mathbf{v}\right) f_{a}(\mathbf{v}) \\
& -\frac{4}{\epsilon^{\prime}\left(\mathbf{k}, \sigma \omega_{\mathbf{k}}^{\alpha}\right)} \sum_{\sigma^{\prime}} \sum_{\beta} \int d \mathbf{k}^{\prime} \operatorname{Im}\left[\mathcal{P} \frac{2\left\{\chi^{(2)}\left(\mathbf{k}^{\prime}, \sigma^{\prime} \omega_{\mathbf{k}^{\prime}}^{\beta} \mid \mathbf{k}-\mathbf{k}^{\prime}, \sigma \omega_{\mathbf{k}}^{\alpha}-\sigma^{\prime} \omega_{\mathbf{k}^{\prime}}^{\beta}\right)\right\}^{2}}{\epsilon\left(\mathbf{k}-\mathbf{k}^{\prime}, \sigma \omega_{\mathbf{k}}^{\alpha}-\sigma^{\prime} \omega_{\mathbf{k}^{\prime}}^{\beta}\right)}\right. \\
& \left.-\bar{\chi}^{(3)}\left(\mathbf{k}^{\prime}, \sigma^{\prime} \omega_{\mathbf{k}^{\prime}}^{\beta}\left|-\mathbf{k}^{\prime},-\sigma^{\prime} \omega_{\mathbf{k}^{\prime}}^{\beta}\right| \mathbf{k}, \sigma \omega_{\mathbf{k}}^{\alpha}\right)\right] I_{\mathbf{k}^{\prime}}^{\sigma^{\prime} \beta} I_{\mathbf{k}}^{\sigma \alpha}-\frac{4 \pi}{\epsilon^{\prime}\left(\mathbf{k}, \sigma \omega_{\mathbf{k}}^{\alpha}\right)} \sum_{\sigma^{\prime}, \sigma^{\prime \prime}} \sum_{\beta, \gamma} \int d \mathbf{k}^{\prime}\left|\chi^{(2)}\left(\mathbf{k}^{\prime}, \sigma^{\prime} \omega_{\mathbf{k}^{\prime}}^{\beta} \mid \mathbf{k}-\mathbf{k}^{\prime}, \sigma^{\prime \prime} \omega_{\mathbf{k}-\mathbf{k}^{\prime}}^{\gamma}\right)\right|^{2} \\
& \times\left[\frac{I_{\mathbf{k}}^{\sigma \alpha} I_{\mathbf{k}-\mathbf{k}^{\prime}}^{\sigma^{\prime \prime} \gamma}}{\epsilon\left(\mathbf{k}^{\prime}, \sigma^{\prime} \omega_{\mathbf{k}^{\prime}}^{\beta}\right)}+\frac{I_{\mathbf{k}}^{\sigma \alpha} I_{\mathbf{k}^{\prime}}^{\sigma^{\prime} \beta}}{\epsilon^{\prime}\left(\mathbf{k}-\mathbf{k}^{\prime}, \sigma^{\prime \prime} \omega_{\mathbf{k}-\mathbf{k}^{\prime}}^{\gamma}\right)}-\frac{I_{\mathbf{k}^{\prime}}^{\sigma^{\prime} \beta} I_{\mathbf{k}-\mathbf{k}^{\prime}}^{\sigma^{\prime \prime} \gamma}}{\epsilon^{\prime}\left(\mathbf{k}, \sigma \omega_{\mathbf{k}}^{\alpha}\right)}\right] \delta\left(\sigma \omega_{\mathbf{k}}^{\alpha}-\sigma^{\prime} \omega_{\mathbf{k}^{\prime}}^{\beta}-\sigma^{\prime \prime} \omega_{\mathbf{k}-\mathbf{k}^{\prime}}^{\gamma}\right) \\
& -\sum_{a} \frac{16 e_{a}^{2}}{\epsilon^{\prime}\left(\mathbf{k}, \sigma \omega_{\mathbf{k}}^{\alpha}\right)} \sum_{\sigma^{\prime}} \sum_{\beta} \int d \mathbf{v} \int d \mathbf{k}^{\prime} \frac{\left|\chi^{(2)}\left(\mathbf{k}^{\prime}, \sigma^{\prime} \omega_{\mathbf{k}^{\prime}}^{\beta} \mid \mathbf{k}-\mathbf{k}^{\prime}, \sigma \omega_{\mathbf{k}}^{\alpha}-\sigma^{\prime} \omega_{\mathbf{k}^{\prime}}^{\beta}\right)\right|^{2}}{\left|\mathbf{k}-\mathbf{k}^{\prime}\right|^{2}\left|\epsilon\left(\mathbf{k}-\mathbf{k}^{\prime}, \sigma \omega_{\mathbf{k}}^{\alpha}-\sigma^{\prime} \omega_{\mathbf{k}^{\prime}}^{\beta}\right)\right|^{2}} \\
& \times\left[\frac{I_{\mathbf{k}}^{\sigma \alpha}}{\epsilon^{\prime}\left(\mathbf{k}^{\prime}, \sigma^{\prime} \omega_{\mathbf{k}^{\prime}}^{\beta}\right)}-\frac{I_{\mathbf{k}^{\prime}}^{\sigma^{\prime} \beta}}{\epsilon^{\prime}\left(\mathbf{k}, \sigma \omega_{\mathbf{k}}^{\alpha}\right)}\right] \delta\left[\sigma \omega_{\mathbf{k}}^{\alpha}-\sigma^{\prime} \omega_{\mathbf{k}^{\prime}}^{\beta}-\left(\mathbf{k}-\mathbf{k}^{\prime}\right) \cdot \mathbf{v}\right] f_{a}(\mathbf{v})-\sum_{a} \frac{8 e_{a}^{2}}{\pi} \frac{1}{\epsilon^{\prime}\left(\mathbf{k}, \sigma \omega_{\mathbf{k}}^{\alpha}\right)} \int d \mathbf{k}^{\prime} \int d \mathbf{v} \frac{1}{k^{\prime 2}\left|\epsilon\left(\mathbf{k}^{\prime}, \mathbf{k}^{\prime} \cdot \mathbf{v}\right)\right|^{2}} \\
& \times \operatorname{Im}\left[\mathcal{P} \frac{2\left\{\chi^{(2)}\left(\mathbf{k}^{\prime}, \mathbf{k}^{\prime} \cdot \mathbf{v} \mid \mathbf{k}-\mathbf{k}^{\prime}, \sigma \omega_{\mathbf{k}}^{\alpha}-\mathbf{k}^{\prime} \cdot \mathbf{v}\right)\right\}^{2}}{\epsilon\left(\mathbf{k}-\mathbf{k}^{\prime}, \sigma \omega_{\mathbf{k}}^{\alpha}-\mathbf{k}^{\prime} \cdot \mathbf{v}\right)}-\bar{\chi}^{(3)}\left(\mathbf{k}^{\prime}, \mathbf{k}^{\prime} \cdot \mathbf{v}\left|-\mathbf{k}^{\prime},-\mathbf{k}^{\prime} \cdot \mathbf{v}\right| \mathbf{k}, \sigma \omega_{\mathbf{k}}^{\alpha}\right)\right] I_{\mathbf{k}}^{\sigma \alpha} f_{a}(\mathbf{v}) \\
& -\sum_{a} \frac{16 e_{a}^{2}}{\epsilon^{\prime}\left(\mathbf{k}, \sigma \omega_{\mathbf{k}}^{\alpha}\right)} \sum_{\sigma^{\prime}} \sum_{\beta} \int d \mathbf{k}^{\prime} \int d \mathbf{v} \frac{\left|\chi^{(2)}\left[\mathbf{k}^{\prime}, \sigma^{\prime} \omega_{\mathbf{k}^{\prime}}^{\beta} \mid \mathbf{k}-\mathbf{k}^{\prime},\left(\mathbf{k}-\mathbf{k}^{\prime}\right) \cdot \mathbf{v}\right]\right|^{2}}{\left|\mathbf{k}-\mathbf{k}^{\prime}\right|^{2}\left|\epsilon\left[\mathbf{k}-\mathbf{k}^{\prime},\left(\mathbf{k}-\mathbf{k}^{\prime}\right) \cdot \mathbf{v}\right]\right|^{2}} \\
& \times\left[\frac{I_{\mathbf{k}}^{\sigma \alpha}}{\epsilon^{\prime}\left(\mathbf{k}^{\prime}, \sigma^{\prime} \omega_{\mathbf{k}^{\prime}}^{\beta}\right)}-\frac{I_{\mathbf{k}^{\prime}}^{\sigma^{\prime} \beta}}{\epsilon^{\prime}\left(\mathbf{k}, \sigma \omega_{\mathbf{k}}^{\alpha}\right)}\right] \delta\left[\sigma \omega_{\mathbf{k}}^{\alpha}-\sigma^{\prime} \omega_{\mathbf{k}^{\prime}}^{\beta}-(\mathbf{k}-\mathbf{k}) \cdot \mathbf{v}\right] f_{a}(\mathbf{v}) \\
& +\sum_{a, b} \frac{48 e_{a}^{2} e_{b}^{2}}{\pi} \frac{1}{\left[\epsilon^{\prime}\left(\mathbf{k}, \sigma \omega_{\mathbf{k}}^{\alpha}\right)\right]^{2}} \int d \mathbf{k}^{\prime} \int d \mathbf{v} \int d \mathbf{v}^{\prime} \frac{\left|\chi^{(2)}\left[\mathbf{k}^{\prime}, \mathbf{k}^{\prime} \cdot \mathbf{v}^{\prime} \mid \mathbf{k}-\mathbf{k}^{\prime},\left(\mathbf{k}-\mathbf{k}^{\prime}\right) \cdot \mathbf{v}\right]\right|^{2}}{k^{\prime 2}\left|\mathbf{k}-\mathbf{k}^{\prime}\right|^{2}\left|\epsilon\left(\mathbf{k}^{\prime}, \mathbf{k}^{\prime} \cdot \mathbf{v}^{\prime}\right)\right|^{2}\left|\epsilon\left[\mathbf{k}-\mathbf{k}^{\prime},\left(\mathbf{k}-\mathbf{k}^{\prime}\right) \cdot \mathbf{v}\right]\right|^{2}} \\
& \times \delta\left[\sigma \omega_{\mathbf{k}}^{\alpha}-\mathbf{k} \cdot \mathbf{v}+\mathbf{k}^{\prime} \cdot\left(\mathbf{v}-\mathbf{v}^{\prime}\right)\right] f_{a}(\mathbf{v}) f_{b}\left(\mathbf{v}^{\prime}\right) .
\end{aligned}
$$

The last three terms on the right-hand side of Eq. (4.24) are new terms associated with noneigenmode fluctuations. In these terms, we encounter various response functions with the angular frequency replaced by $\mathbf{k} \cdot \mathbf{v}$. We make use of the approximation (4.17), namely, $\epsilon(\mathbf{k}, \mathbf{k} \cdot \mathbf{v}) \approx \epsilon(\mathbf{k}, 0)=1+\left(2 \omega_{p e}^{2} / k^{2} v_{T e}^{2}\right)\left(1+T_{e} / T_{i}\right)$, which was used to simplify the collision integral for the particles, 
Eq. (4.18). Recall that further approximation, $\epsilon(\mathbf{k}, 0) \approx 1$ leads to the reduction of the Balescu-Lénard collision integral to Landau collision integral, which physically implies particle interacting through Debye-screened collisional processes. However, here we do not assume $k^{2} \lambda_{D e}^{2} \gg 1$, because the waves are not subject to Debye screening. We then have

$$
\begin{aligned}
& \frac{\partial I_{\mathbf{k}}^{\sigma \alpha}}{\partial t}=-\frac{2 \operatorname{Im} \epsilon\left(\mathbf{k}, \sigma \omega_{\mathbf{k}}^{\alpha}\right)}{\epsilon^{\prime}\left(\mathbf{k}, \sigma \omega_{\mathbf{k}}^{\alpha}\right)} I_{\mathbf{k}}^{\sigma \alpha}+\sum_{a} \frac{4 e_{a}^{2}}{k^{2}\left[\epsilon^{\prime}\left(\mathbf{k}, \sigma \omega_{\mathbf{k}}^{\alpha}\right)\right]^{2}} \int d \mathbf{v} \delta\left(\sigma \omega_{\mathbf{k}}^{\alpha}-\mathbf{k} \cdot \mathbf{v}\right) f_{a}(\mathbf{v}) \\
& -\frac{4 \pi}{\epsilon^{\prime}\left(\mathbf{k}, \sigma \omega_{\mathbf{k}}^{\alpha}\right)} \sum_{\sigma^{\prime}, \sigma^{\prime \prime}} \sum_{\beta, \gamma} \int d \mathbf{k}^{\prime}\left|\chi^{(2)}\left(\mathbf{k}^{\prime}, \sigma^{\prime} \omega_{\mathbf{k}^{\prime}}^{\beta} \mid \mathbf{k}-\mathbf{k}^{\prime}, \sigma^{\prime \prime} \omega_{\mathbf{k}-\mathbf{k}^{\prime}}^{\gamma}\right)\right|^{2} \\
& \times\left[\frac{I_{\mathbf{k}}^{\sigma \alpha} I_{\mathbf{k}-\mathbf{k}^{\prime}}^{\sigma^{\prime \prime} \gamma}}{\epsilon\left(\mathbf{k}^{\prime}, \sigma^{\prime} \omega_{\mathbf{k}^{\prime}}^{\beta}\right)}+\frac{I_{\mathbf{k}}^{\sigma \alpha} I_{\mathbf{k}^{\prime}}^{\sigma^{\prime} \beta}}{\epsilon^{\prime}\left(\mathbf{k}-\mathbf{k}^{\prime}, \sigma^{\prime \prime} \omega_{\mathbf{k}-\mathbf{k}^{\prime}}^{\gamma}\right)}-\frac{I_{\mathbf{k}^{\prime}}^{\sigma^{\prime} \beta} I_{\mathbf{k}-\mathbf{k}^{\prime}}^{\sigma^{\prime \prime} \gamma}}{\epsilon^{\prime}\left(\mathbf{k}, \sigma \omega_{\mathbf{k}}^{\alpha}\right)}\right] \delta\left(\sigma \omega_{\mathbf{k}}^{\alpha}-\sigma^{\prime} \omega_{\mathbf{k}^{\prime}}^{\beta}-\sigma^{\prime \prime} \omega_{\mathbf{k}-\mathbf{k}^{\prime}}^{\gamma}\right) \\
& -\frac{4}{\epsilon^{\prime}\left(\mathbf{k}, \sigma \omega_{\mathbf{k}}^{\alpha}\right)} \sum_{\sigma^{\prime}} \sum_{\beta} \int d \mathbf{k}^{\prime} \operatorname{Im}\left[\mathcal{P} \frac{2\left\{\chi^{(2)}\left(\mathbf{k}^{\prime}, \sigma^{\prime} \omega_{\mathbf{k}^{\prime}}^{\beta} \mid \mathbf{k}-\mathbf{k}^{\prime}, \sigma \omega_{\mathbf{k}}^{\alpha}-\sigma^{\prime} \omega_{\mathbf{k}^{\prime}}^{\beta}\right)\right\}^{2}}{\epsilon\left(\mathbf{k}-\mathbf{k}^{\prime}, \sigma \omega_{\mathbf{k}}^{\alpha}-\sigma^{\prime} \omega_{\mathbf{k}^{\prime}}^{\beta}\right)}\right. \\
& \left.-\bar{\chi}^{(3)}\left(\mathbf{k}^{\prime}, \sigma^{\prime} \omega_{\mathbf{k}^{\prime}}^{\beta}\left|-\mathbf{k}^{\prime},-\sigma^{\prime} \omega_{\mathbf{k}^{\prime}}^{\beta}\right| \mathbf{k}, \sigma \omega_{\mathbf{k}}^{\alpha}\right)\right] I_{\mathbf{k}^{\prime}}^{\sigma^{\prime} \beta} I_{\mathbf{k}}^{\sigma \alpha} \\
& -\sum_{a} \frac{16 e_{a}^{2}}{\epsilon^{\prime}\left(\mathbf{k}, \sigma \omega_{\mathbf{k}}^{\alpha}\right)} \sum_{\sigma^{\prime}} \sum_{\beta} \int d \mathbf{v} \int \frac{d \mathbf{k}^{\prime}}{\left|\mathbf{k}-\mathbf{k}^{\prime}\right|^{2}}\left[\frac{\left|\chi^{(2)}\left(\mathbf{k}^{\prime}, \sigma^{\prime} \omega_{\mathbf{k}^{\prime}}^{\beta} \mid \mathbf{k}-\mathbf{k}^{\prime}, \sigma \omega_{\mathbf{k}}^{\alpha}-\sigma^{\prime} \omega_{\mathbf{k}^{\prime}}^{\beta}\right)\right|^{2}}{\left|\epsilon\left(\mathbf{k}-\mathbf{k}^{\prime}, \sigma \omega_{\mathbf{k}}^{\alpha}-\sigma^{\prime} \omega_{\mathbf{k}^{\prime}}^{\beta}\right)\right|^{2}}\right. \\
& +\underbrace{\left.\frac{\left|\chi^{(2)}\left(\mathbf{k}^{\prime}, \sigma^{\prime} \omega_{\mathbf{k}^{\prime}}^{\beta} \mid \mathbf{k}-\mathbf{k}^{\prime}, 0\right)\right|^{2}}{\left|\epsilon\left(\mathbf{k}-\mathbf{k}^{\prime}, 0\right)\right|^{2}}\right]}\left[\frac{I_{\mathbf{k}}^{\sigma \alpha}}{\epsilon^{\prime}\left(\mathbf{k}^{\prime}, \sigma^{\prime} \omega_{\mathbf{k}^{\prime}}^{\beta}\right)}-\frac{I_{\mathbf{k}^{\prime}}^{\sigma^{\prime} \beta}}{\epsilon^{\prime}\left(\mathbf{k}, \sigma \omega_{\mathbf{k}}^{\alpha}\right)}\right] \delta\left[\sigma \omega_{\mathbf{k}}^{\alpha}-\sigma^{\prime} \omega_{\mathbf{k}^{\prime}}^{\beta}-\left(\mathbf{k}-\mathbf{k}^{\prime}\right) \cdot \mathbf{v}\right] f_{a}(\mathbf{v}) \\
& -\left\{\sum_{a} \frac{8 e_{a}^{2}}{\pi} \frac{1}{\epsilon^{\prime}\left(\mathbf{k}, \sigma \omega_{\mathbf{k}}^{\alpha}\right)} \int \frac{d \mathbf{k}^{\prime}}{k^{\prime 2}\left|\epsilon\left(\mathbf{k}^{\prime}, 0\right)\right|^{2}} \int d \mathbf{v} \operatorname{Im}\left[\mathcal{P} \frac{2\left\{\chi^{(2)}\left(\mathbf{k}^{\prime}, 0 \mid \mathbf{k}-\mathbf{k}^{\prime}, \sigma \omega_{\mathbf{k}}^{\alpha}\right)\right\}^{2}}{\epsilon\left(\mathbf{k}-\mathbf{k}^{\prime}, \sigma \omega_{\mathbf{k}}^{\alpha}\right)}-\bar{\chi}^{(3)}\left(\mathbf{k}^{\prime}, 0\left|-\mathbf{k}^{\prime}, 0\right| \mathbf{k}, \sigma \omega_{\mathbf{k}}^{\alpha}\right)\right] f_{a}(\mathbf{v}) I_{\mathbf{k}}^{\sigma \alpha}\right\}_{\mathrm{A}} \\
& +\left\{\sum_{a, b} \frac{48 e_{a}^{2} e_{b}^{2}}{\pi} \frac{1}{\left[\epsilon^{\prime}\left(\mathbf{k}, \sigma \omega_{\mathbf{k}}^{\alpha}\right)\right]^{2}} \int d \mathbf{k}^{\prime} \int d \mathbf{v} \int d \mathbf{v}^{\prime} \frac{\left|\chi^{(2)}\left(\mathbf{k}^{\prime}, 0 \mid \mathbf{k}-\mathbf{k}^{\prime}, 0\right)\right|^{2}}{k^{\prime 2}\left|\mathbf{k}-\mathbf{k}^{\prime}\right|^{2}\left|\epsilon\left(\mathbf{k}^{\prime}, 0\right)\right|^{2}\left|\epsilon\left(\mathbf{k}-\mathbf{k}^{\prime}, 0\right)\right|^{2}}\right. \\
& \left.\times \delta\left[\sigma \omega_{\mathbf{k}}^{\alpha}-\mathbf{k} \cdot \mathbf{v}+\mathbf{k}^{\prime} \cdot\left(\mathbf{v}-\mathbf{v}^{\prime}\right)\right] f_{a}(\mathbf{v}) f_{b}\left(\mathbf{v}^{\prime}\right)\right\}_{\mathrm{B}} .
\end{aligned}
$$

In the above

$$
\epsilon(\mathbf{k}, 0)=1+\left(1+\frac{T_{e}}{T_{i}}\right) \frac{1}{k^{2} \lambda_{D e}^{2}} .
$$

It is seen that the contributions from noneigenmodes lead to three new effects. One is the term indicated by the underbrace

which is a new term that arises from the noneigenmode fluctuation, and this term has a net effect of modifying the spontaneous scattering term. The second term contained within the large square brackets and indicated by subscript " $\mathrm{A}$ " exhibits the overall dependence on the wave intensity, $I_{\mathbf{k}}^{\sigma \alpha}$. This term thus has the structure that is representative of the collisional damping term. The final term has the appearance of the electrostatic bremsstrahlung, which describes the emission of electrostatic fluctuation in the eigenmode regime, $\omega=\sigma \omega_{\mathbf{k}}^{\alpha}$, but its causation is the particle scattering (hence, the "breaking radiation").

Here we should caution readers that the term "electrostatic bremsstrahlung" is not to be confused with a process sometimes known in the literature by the same terminology, which is unfortunate. In the literature, the process of relativistic electrons scattering Langmuir waves into transverse radiation is also called the "electrostatic bremsstrahlung" [53-58]. However, this process, which should be more accurately called the "inverse plasmon scattering" [56], is actually an induced scattering of transverse radiation off relativistic electrons mediated by Langmuir waves. In contrast, the present "electrostatic bremsstrahlung" is an emission of electrostatic eigenmodes by collisional process, which is analogous to the emission of transverse electromagnetic radiation by collisional process. Note that several authors discussed the electromagnetic bremsstrahlung within the context of plasma kinetic theory [59-62] but a similar process involving electrostatic modes has never been discussed in the literature.

Concerning the collisional damping rate, we should also caution the readers that the present formulation differs from that customarily found in the literature. As noted in the Introduction, one of the customary approaches to discuss collisional damping of plasma waves is to simply add the collisional operator to the particle kinetic equation in an ad hoc manner [42]; see Eq. (1.5). Upon replacing the full collision integral by an effective collision frequency and upon linearizing the particle equation, the particle effective collision frequency gets absorbed to the wave-particle resonance condition. In this approach, the particle collision frequency automatically gets translated as the wave collision frequency. 
A slightly more rigorous discussion of the collisional damping formula can found, e.g., in a monograph by Melrose [8], according to which, first, the bremsstrahlung radiation formula is derived by considering the single particle dynamics. The essential formula for the radiation power is generalized to an ensemble of charged particles by multiplying the single-particle formula by the particle number density distribution [see Eq. (3.81) of Ref. [8], Vol. 1]. Then, the Kirchoff's law, which in the parlance of plasma kinetic theory is none other than the balance of the right-hand side of the quasilinear wave kinetic equation that describes emission and reabsorption of waves [see Eq. (5.19) of Ref. [8], Vol. 1], is invoked in order to deduce the collisional damping rate of the radiation [see Eq. (6.3) of Ref. [8], Vol. 1]. Adopting such a formula for the collisional damping Ref. [38] includes the collisional damping of Langmuir waves in their discussion of wave-particle interactions of the solar flare electron context; see Eq. (3) of Ref. [38]. However, strictly speaking, the collisional damping rate discussed in Ref. [8] rigorously applies to the transverse radiation only. Also, simply invoking the Kirchoff's law to determine the collisional damping rate is not entirely self-consistent. In contrast, the present formalism actually derives the Kirchoff law plus other terms within the wave kinetic equation.

The third new effect, namely, the modification of the spontaneous scattering term via noneigenmode contributions has not been discussed in the literature. The ramifications and actual applications of the present improved weak turbulence formalism will be carried out in the future, but for the moment, the subsequent exposition is by necessity, formal.

\section{Electrostatic bremsstrahlung}

The electrostatic bremmstrahlung terms for $L$ and $S$ mode wave kinetic equations come from term B in Eq. (4.25):

$$
\begin{aligned}
& \left.\frac{\partial I_{\mathbf{k}}^{\sigma L}}{\partial t}\right|_{\text {bremss }}=\sum_{a, b} \frac{12 \hat{n}^{2} e_{a}^{2} e_{b}^{2} \omega_{p e}^{2}}{\pi} \int d \mathbf{k}^{\prime} \int d \mathbf{v} \int d \mathbf{v}^{\prime} \frac{\left|\chi^{(2)}\left(\mathbf{k}^{\prime}, 0 \mid \mathbf{k}-\mathbf{k}^{\prime}, 0\right)\right|^{2}}{k^{\prime 2}\left|\mathbf{k}-\mathbf{k}^{\prime}\right|^{2}\left|\epsilon\left(\mathbf{k}^{\prime}, 0\right)\right|^{2}\left|\epsilon\left(\mathbf{k}-\mathbf{k}^{\prime}, 0\right)\right|^{2}} \delta\left[\sigma \omega_{\mathbf{k}}^{L}-\mathbf{k} \cdot \mathbf{v}+\mathbf{\mathbf { k } ^ { \prime }} \cdot\left(\mathbf{v}-\mathbf{v}^{\prime}\right)\right] F_{a}(\mathbf{v}) F_{b}\left(\mathbf{v}^{\prime}\right), \\
& \left.\frac{\partial I_{\mathbf{k}}^{\sigma S}}{\partial t}\right|_{\text {bremss }}=\sum_{a, b} \frac{12 \hat{n}^{2} e_{a}^{2} e_{b}^{2} \omega_{p e}^{2}}{\pi} \mu_{\mathbf{k}} \int d \mathbf{k}^{\prime} \int d \mathbf{v} \int d \mathbf{v}^{\prime} \frac{\left|\chi^{(2)}\left(\mathbf{k}^{\prime}, 0 \mid \mathbf{k}-\mathbf{k}^{\prime}, 0\right)\right|^{2}}{k^{\prime 2}\left|\mathbf{k}-\mathbf{k}^{\prime}\right|^{2}\left|\epsilon\left(\mathbf{k}^{\prime}, 0\right)\right|^{2}\left|\epsilon\left(\mathbf{k}-\mathbf{k}^{\prime}, 0\right)\right|^{2}} \delta\left[\sigma \omega_{\mathbf{k}}^{S}-\mathbf{k} \cdot \mathbf{v}+\mathbf{k}^{\prime} \cdot\left(\mathbf{v}-\mathbf{v}^{\prime}\right)\right] F_{a}(\mathbf{v}) F_{b}\left(\mathbf{v}^{\prime}\right) .
\end{aligned}
$$

Upon making use of Eq. (3.12), we make the following approximation:

$$
\chi^{(2)}\left(\mathbf{k}^{\prime}, 0 \mid \mathbf{k}-\mathbf{k}^{\prime}, 0\right)=-i \sum_{a} \frac{e_{a}}{T_{a}} \frac{\omega_{p a}^{2}}{k k^{\prime}\left|\mathbf{k}-\mathbf{k}^{\prime}\right|} \frac{1}{v_{T a}^{2}}=\frac{i e}{T_{e}} \frac{1}{v_{T e}^{2}}\left(1-\frac{T_{e}^{2}}{T_{i}^{2}}\right) \frac{\omega_{p e}^{2}}{k k^{\prime}\left|\mathbf{k}-\mathbf{k}^{\prime}\right|} .
$$

This leads to the simplification of bremsstrahlung terms in Eq. (4.25) for $\alpha=L$ and $\alpha=S$,

$$
\begin{aligned}
\left.\frac{\partial I_{\mathbf{k}}^{\sigma L}}{\partial t}\right|_{\text {bremss }}= & \frac{3 e^{2} T_{e}}{16 \pi^{3} m_{e}}\left(1-\frac{T_{e}^{2}}{T_{i}^{2}}\right)^{2} \frac{1}{k^{2} \lambda_{D e}^{2}} \int d \mathbf{k}^{\prime}\left(1+\frac{T_{e}}{T_{i}}+k^{\prime 2} \lambda_{D e}^{2}\right)^{-2}\left[1+\frac{T_{e}}{T_{i}}+\left(\mathbf{k}-\mathbf{k}^{\prime}\right)^{2} \lambda_{D e}^{2}\right]^{-2} \\
& \times \int d \mathbf{v} \int d \mathbf{v} \delta\left[\sigma \omega_{\mathbf{k}}^{L}-\mathbf{k} \cdot \mathbf{v}+\mathbf{k}^{\prime} \cdot\left(\mathbf{v}-\mathbf{v}^{\prime}\right)\right] \sum_{a} F_{a}(\mathbf{v}) \sum_{b} F_{b}\left(\mathbf{v}^{\prime}\right), \\
\left.\frac{\partial I_{\mathbf{k}}^{\sigma S}}{\partial t}\right|_{\text {bremss }}= & \frac{3 e^{2} T_{e}}{16 \pi^{3} m_{e}}\left(1-\frac{T_{e}^{2}}{T_{i}^{2}}\right)^{2} \frac{\mu_{\mathbf{k}}}{k^{2} \lambda_{D e}^{2}} \int d \mathbf{k}^{\prime}\left(1+\frac{T_{e}}{T_{i}}+k^{\prime 2} \lambda_{D e}^{2}\right)^{-2}\left[1+\frac{T_{e}}{T_{i}}+\left(\mathbf{k}-\mathbf{k}^{\prime}\right)^{2} \lambda_{D e}^{2}\right]^{-2} \\
& \times \int d \mathbf{v} \int d \mathbf{v} \delta\left[\sigma \omega_{\mathbf{k}}^{S}-\mathbf{k} \cdot \mathbf{v}+\mathbf{k}^{\prime} \cdot\left(\mathbf{v}-\mathbf{v}^{\prime}\right)\right] \sum_{a} F_{a}(\mathbf{v}) \sum_{b} F_{b}\left(\mathbf{v}^{\prime}\right) .
\end{aligned}
$$

\section{Noneigenmode correction to spontaneous scattering}

The noneigenmode corrections to the spontaneous scattering comes from the term indicated by the underbrace in Eq. (4.25):

$$
\begin{aligned}
\left.\frac{\partial I_{\mathbf{k}}^{\sigma L}}{\partial t}\right|_{\text {corr }}= & -4 \hat{n} e^{2} \sum_{\sigma^{\prime}} \int d \mathbf{k}^{\prime} \int d \mathbf{v} \frac{\left|\chi^{(2)}\left(\mathbf{k}^{\prime}, \sigma^{\prime} \omega_{\mathbf{k}^{\prime}}^{L} \mid \mathbf{k}-\mathbf{k}^{\prime}, 0\right)\right|^{2}}{\left|\mathbf{k}-\mathbf{k}^{\prime}\right|^{2}|\epsilon(\mathbf{k}-\mathbf{k}, 0)|^{2}} \\
& \times \sigma \omega_{\mathbf{k}}^{L}\left(\sigma^{\prime} \omega_{\mathbf{k}^{\prime}}^{L} I_{\mathbf{k}}^{\sigma L}-\sigma \omega_{\mathbf{k}}^{L} I_{\mathbf{k}^{\prime}}^{\sigma^{\prime} L}\right) \delta\left[\sigma \omega_{\mathbf{k}}^{L}-\sigma^{\prime} \omega_{\mathbf{k}^{\prime}}^{L}-\left(\mathbf{k}-\mathbf{k}^{\prime}\right) \cdot \mathbf{v}\right] \sum_{a} F_{a}(\mathbf{v}), \\
\left.\frac{\partial I_{\mathbf{k}}^{\sigma S}}{\partial t}\right|_{\text {corr }}= & -4 \hat{n} e^{2} \mu_{\mathbf{k}} \mu_{\mathbf{k}^{\prime}} \sigma \omega_{\mathbf{k}}^{L} \sum_{\sigma^{\prime}} \int d \mathbf{k}^{\prime} \int d \mathbf{v} \frac{\left|\chi^{(2)}\left(\mathbf{k}^{\prime}, \sigma^{\prime} \omega_{\mathbf{k}^{\prime}}^{S} \mid \mathbf{k}-\mathbf{k}^{\prime}, 0\right)\right|^{2}}{\left|\mathbf{k}-\mathbf{k}^{\prime}\right|^{2}|\epsilon(\mathbf{k}-\mathbf{k}, 0)|^{2}} \\
& \times\left(\sigma^{\prime} \omega_{\mathbf{k}^{\prime}}^{L} I_{\mathbf{k}}^{\sigma S}-\sigma \omega_{\mathbf{k}}^{L} I_{\mathbf{k}^{\prime}}^{\sigma^{\prime} S}\right) \delta\left[\sigma \omega_{\mathbf{k}}^{S}-\sigma^{\prime} \omega_{\mathbf{k}^{\prime}}^{S}-\left(\mathbf{k}-\mathbf{k}^{\prime}\right) \cdot \mathbf{v}\right] \sum_{a} F_{a}(\mathbf{v}) .
\end{aligned}
$$

For the second-order susceptibility $\chi^{(2)}\left(\mathbf{k}^{\prime}, \sigma^{\prime} \omega_{\mathbf{k}^{\prime}}^{L} / \mathbf{k}-\mathbf{k}^{\prime}, 0\right)$ we may assume $\omega_{\mathbf{k}^{\prime}}^{L} \gg k^{\prime} v_{T e}$ and $\omega_{\mathbf{k}^{\prime}}^{L} \gg k^{\prime} v_{T i}$. For $\chi^{(2)}\left(\mathbf{k}^{\prime}, \sigma^{\prime} \omega_{\mathbf{k}^{\prime}}^{S} \mid \mathbf{k}-\mathbf{k}^{\prime}, 0\right)$, the approximation involves $\omega_{\mathbf{k}^{\prime}}^{S} \ll k^{\prime} v_{T e}$ and $\omega_{\mathbf{k}^{\prime}}^{S} \gg k^{\prime} v_{T i}$. The second-order nonlinear susceptibility 
for $L$ mode, $\chi^{(2)}\left(\mathbf{k}^{\prime}, \sigma^{\prime} \omega_{\mathbf{k}^{\prime}}^{L} \mid \mathbf{k}-\mathbf{k}^{\prime}, 0\right)$, and the ion partial susceptibility for $S$ mode, $\chi_{i}^{(2)}\left(\mathbf{k}^{\prime}, \sigma^{\prime} \omega_{\mathbf{k}^{\prime}}^{S} \mid \mathbf{k}-\mathbf{k}^{\prime}, 0\right)$, lend themselves to the well-known approximation, namely,

$$
\begin{aligned}
& \chi^{(2)}\left(\mathbf{k}^{\prime}, \sigma^{\prime} \omega_{\mathbf{k}^{\prime}}^{L} \mid \mathbf{k}-\mathbf{k}^{\prime}, 0\right) \approx \chi_{e}^{(2)}\left(\mathbf{k}^{\prime}, \sigma^{\prime} \omega_{\mathbf{k}^{\prime}}^{L} \mid \mathbf{k}-\mathbf{k}^{\prime}, 0\right)=\frac{-i}{2} \frac{e}{T_{e}} \frac{\mathbf{k} \cdot \mathbf{k}^{\prime}}{k k^{\prime}\left|\mathbf{k}-\mathbf{k}^{\prime}\right|}, \\
& \chi_{i}^{(2)}\left(\mathbf{k}^{\prime}, \sigma^{\prime} \omega_{\mathbf{k}^{\prime}}^{S} \mid \mathbf{k}-\mathbf{k}^{\prime}, 0\right)=\frac{-i e}{2 T_{i}} \frac{1}{k k^{\prime}\left|\mathbf{k}-\mathbf{k}^{\prime}\right| \lambda_{D e}^{2}} \frac{\mathbf{k} \cdot \mathbf{k}^{\prime}}{k^{\prime 2}} .
\end{aligned}
$$

However, to compute the electron partial susceptibility $\chi_{e}^{(2)}\left(\mathbf{k}^{\prime}, \sigma^{\prime} \omega_{\mathbf{k}^{\prime}}^{S} / \mathbf{k}-\mathbf{k}^{\prime}, 0\right)$ requires fundamental reconsideration since in this case, both arguments representing angular frequencies are slow modes. We thus manipulate the original definition (2.40) and write

$$
\chi_{e}^{(2)}\left(\mathbf{k}^{\prime}, \sigma^{\prime} \omega_{\mathbf{k}^{\prime}}^{S} \mid \mathbf{k}-\mathbf{k}^{\prime}, 0\right)=\frac{i e}{2 m_{e}} \frac{\omega_{p e}^{2}}{k k^{\prime}\left|\mathbf{k}-\mathbf{k}^{\prime}\right|} \int d \mathbf{v} \frac{1}{\sigma^{\prime} \omega_{\mathbf{k}^{\prime}}^{S}-\mathbf{k} \cdot \mathbf{v}+i 0}\left[\mathbf{k}^{\prime} \cdot \frac{\partial}{\partial \mathbf{v}} \frac{\left(\mathbf{k}-\mathbf{k}^{\prime}\right) \cdot \partial F_{e} / \partial \mathbf{v}}{-\left(\mathbf{k}-\mathbf{k}^{\prime}\right) \cdot \mathbf{v}+i 0}+\left(\mathbf{k}-\mathbf{k}^{\prime}\right) \cdot \frac{\partial}{\partial \mathbf{v}} \frac{\mathbf{k}^{\prime} \cdot \partial F_{e} / \partial \mathbf{v}}{\sigma^{\prime} \omega_{\mathbf{k}^{\prime}}^{S}-\mathbf{k}^{\prime} \cdot \mathbf{v}+i 0}\right]
$$

Upon assuming that the bulk electron population can be approximated by a Gaussian distribution we may trivially carry out the velocity integrations. Since $\omega_{\mathbf{k}^{\prime}}^{S} \ll k^{\prime} v_{T e}$, we may ignore $\omega_{\mathbf{k}^{\prime}}^{S}$ in comparison with $\mathbf{k}^{\prime} \cdot \mathbf{v}$. This readily leads to

$$
\chi_{e}^{(2)}\left(\mathbf{k}^{\prime}, \sigma^{\prime} \omega_{\mathbf{k}^{\prime}}^{S} \mid \mathbf{k}-\mathbf{k}^{\prime}, 0\right)=\frac{i e}{2 T_{e}} \frac{1}{k k^{\prime}\left|\mathbf{k}-\mathbf{k}^{\prime}\right| \lambda_{D e}^{2}} .
$$

Gathering all the results, namely, Eqs. (4.31) and (4.33), the correction term to the spontaneous scattering effects that arises from noneigenmodes, Eq. (4.25), is now given by

$$
\begin{aligned}
\left.\frac{\partial I_{\mathbf{k}}^{\sigma L}}{\partial t}\right|_{\text {corr }}= & -\frac{\hat{n} e^{4}}{m_{e}^{2} \omega_{p e}^{4}} \sigma \omega_{\mathbf{k}}^{L} \sum_{\sigma^{\prime}} \int d \mathbf{k}^{\prime} \int d \mathbf{v} \frac{\left(\mathbf{k} \cdot \mathbf{k}^{\prime}\right)^{2}}{k^{2} k^{\prime 2}} \delta\left[\sigma \omega_{\mathbf{k}}^{L}-\sigma^{\prime} \omega_{\mathbf{k}^{\prime}}^{L}-\left(\mathbf{k}-\mathbf{k}^{\prime}\right) \cdot \mathbf{v}\right] \\
& \times\left[1+\frac{T_{e}}{T_{i}}+\left(\mathbf{k}-\mathbf{k}^{\prime}\right)^{2} \lambda_{D e}^{2}\right]^{-2}\left(\sigma^{\prime} \omega_{\mathbf{k}^{\prime}}^{L} I_{\mathbf{k}}^{\sigma L}-\sigma \omega_{\mathbf{k}}^{L} I_{\mathbf{k}^{\prime}}^{\sigma^{\prime} L}\right) \sum_{a} F_{a}(\mathbf{v}), \\
\left.\frac{\partial I_{\mathbf{k}}^{\sigma S}}{\partial t}\right|_{\text {corr }}= & -\frac{\hat{n} e^{4}}{m_{e}^{2} \omega_{p e}^{4}} \mu_{\mathbf{k}} \mu_{\mathbf{k}^{\prime}} \sigma \omega_{\mathbf{k}}^{L} \sum_{\sigma^{\prime}} \int d \mathbf{k}^{\prime} \int d \mathbf{v} \frac{1}{k^{2} k^{\prime 2} \lambda_{D e}^{4}} \delta\left[\sigma \omega_{\mathbf{k}}^{S}-\sigma^{\prime} \omega_{\mathbf{k}^{\prime}}^{S}-\left(\mathbf{k}-\mathbf{k}^{\prime}\right) \cdot \mathbf{v}\right] \\
& \times\left[1+\frac{T_{e}}{T_{i}}+\left(\mathbf{k}-\mathbf{k}^{\prime}\right)^{2} \lambda_{D e}^{2}\right]^{-2}\left(1-\frac{T_{e}}{T_{i}} \frac{\mathbf{k} \cdot \mathbf{k}^{\prime}}{k^{\prime 2}}\right)^{2}\left(\sigma^{\prime} \omega_{\mathbf{k}^{\prime}}^{L} I_{\mathbf{k}}^{\sigma S}-\sigma \omega_{\mathbf{k}}^{L} I_{\mathbf{k}^{\prime}}^{\sigma^{\prime} S}\right) \sum_{a} F_{a}(\mathbf{v}) .
\end{aligned}
$$

\section{Collisional damping}

The terms in the wave kinetic equation (4.25) which describe collisional damping are given by the new term designated by subscript A:

$$
\begin{aligned}
& \left.\frac{\partial I_{\mathbf{k}}^{\sigma L}}{\partial t}\right|_{\text {coll }}=-\sum_{a} \frac{4 \hat{n} e_{a}^{2}}{\pi} \sigma \omega_{\mathbf{k}}^{L} \int \frac{d \mathbf{k}^{\prime}}{k^{\prime 2}\left|\epsilon\left(\mathbf{k}^{\prime}, 0\right)\right|^{2}} \int d \mathbf{v} \operatorname{Im}\left[\mathcal{P} \frac{2\left\{\chi^{(2)}\left(\mathbf{k}^{\prime}, 0 \mid \mathbf{k}-\mathbf{k}^{\prime}, \sigma \omega_{\mathbf{k}}^{L}\right)\right\}^{2}}{\epsilon\left(\mathbf{k}-\mathbf{k}^{\prime}, \sigma \omega_{\mathbf{k}}^{L}\right)}-\bar{\chi}^{(3)}\left(\mathbf{k}^{\prime}, 0\left|-\mathbf{k}^{\prime}, 0\right| \mathbf{k}, \sigma \omega_{\mathbf{k}}^{L}\right)\right] F_{a}(\mathbf{v}) I_{\mathbf{k}}^{\sigma L}, \\
& \left.\frac{\partial I_{\mathbf{k}}^{\sigma S}}{\partial t}\right|_{\text {coll }}=-\sum_{a} \frac{4 \hat{n} e_{a}^{2}}{\pi} \sigma \mu_{\mathbf{k}} \omega_{\mathbf{k}}^{L} \int \frac{d \mathbf{k}^{\prime}}{k^{\prime 2}\left|\epsilon\left(\mathbf{k}^{\prime}, 0\right)\right|^{2}} \int d \mathbf{v} \operatorname{Im}\left[\mathcal{P} \frac{2\left\{\chi^{(2)}\left(\mathbf{k}^{\prime}, 0 \mid \mathbf{k}-\mathbf{k}^{\prime}, \sigma \omega_{\mathbf{k}}^{S}\right)\right\}^{2}}{\epsilon\left(\mathbf{k}-\mathbf{k}^{\prime}, \sigma \omega_{\mathbf{k}}^{S}\right)}-\bar{\chi}^{(3)}\left(\mathbf{k}^{\prime}, 0\left|-\mathbf{k}^{\prime}, 0\right| \mathbf{k}, \sigma \omega_{\mathbf{k}}^{S}\right)\right] F_{a}(\mathbf{v}) I_{\mathbf{k}}^{\sigma S} .
\end{aligned}
$$

The collisional damping terms above are associated with the following approximate forms of the nonlinear susceptibilities:

$$
\begin{aligned}
\chi^{(2)}\left(\mathbf{k}^{\prime}, 0 \mid \mathbf{k}-\mathbf{k}^{\prime}, \sigma \omega_{\mathbf{k}}^{L}\right) & \approx \chi_{e}^{(2)}\left(\mathbf{k}^{\prime}, 0 \mid \mathbf{k}-\mathbf{k}^{\prime}, \sigma \omega_{\mathbf{k}}^{L}\right)=\frac{-i e}{T_{e}} \frac{\mathbf{k} \cdot\left(\mathbf{k}-\mathbf{k}^{\prime}\right)}{k k^{\prime}\left|\mathbf{k}-\mathbf{k}^{\prime}\right|}, \\
\chi_{e}^{(2)}\left(\mathbf{k}^{\prime}, 0 \mid \mathbf{k}-\mathbf{k}^{\prime}, \sigma \omega_{\mathbf{k}}^{S}\right) & =\frac{i e}{2 T_{e}} \frac{1}{k k^{\prime}\left|\mathbf{k}-\mathbf{k}^{\prime}\right| \lambda_{D e}^{2}} \\
\chi_{i}^{(2)}\left(\mathbf{k}^{\prime}, 0 \mid \mathbf{k}-\mathbf{k}^{\prime}, \sigma \omega_{\mathbf{k}}^{S}\right) & =\frac{i e}{T_{i}} \frac{1}{k k^{\prime}\left|\mathbf{k}-\mathbf{k}^{\prime}\right| \lambda_{D e}^{2}} \frac{\mathbf{k} \cdot\left(\mathbf{k}-\mathbf{k}^{\prime}\right)}{k^{2}}
\end{aligned}
$$

For the third-order susceptibility, in the case of $\omega=\sigma \omega_{\mathbf{k}}^{L}$, both electron and ion susceptibilities pertain to two slow modes and one fast mode, for which we do not have standard approximate formula [see Eq. (3.13)]. We thus write down the specific 
expression on the basis of the general definition (2.41):

$$
\begin{aligned}
\bar{\chi}_{a}^{(3)}\left(\mathbf{k}^{\prime}, 0\left|-\mathbf{k}^{\prime}, 0\right| \mathbf{k}, \omega\right)= & \frac{1}{2} \frac{e_{a}^{2}}{m_{a}^{2}} \frac{\omega_{p a}^{2}}{k^{2} k^{\prime 2}} \int d \mathbf{v} \frac{1}{\omega-\mathbf{k} \cdot \mathbf{v}+i 0} \mathbf{k}^{\prime} \\
& \cdot \frac{\partial}{\partial \mathbf{v}}\left\{\frac{1}{\omega-\left(\mathbf{k}-\mathbf{k}^{\prime}\right) \cdot \mathbf{v}+i 0}\left[\mathbf{k}^{\prime} \cdot \frac{\partial}{\partial \mathbf{v}}\left(\frac{\mathbf{k} \cdot \partial F_{a} / \partial \mathbf{v}}{\omega-\mathbf{k} \cdot \mathbf{v}+i 0}\right)+\mathbf{k} \cdot \mathbf{v}\left(\frac{2}{v_{T a}^{2}}\right)^{2} F_{a}\right]\right\} .
\end{aligned}
$$

For $\omega=\sigma \omega_{\mathbf{k}}^{L}$ we have

$$
\begin{aligned}
& \bar{\chi}_{e}^{(3)}\left(\mathbf{k}^{\prime}, 0\left|-\mathbf{k}^{\prime}, 0\right| \mathbf{k}, \sigma \omega_{\mathbf{k}}^{L}\right)=\frac{1}{2} \frac{e^{2}}{m_{e}^{2}} \frac{1}{k^{2}{k^{\prime}}^{\prime 2}} \int d \mathbf{v} \mathbf{k}^{\prime} \cdot \frac{\partial}{\partial \mathbf{v}}\left[\mathbf{k}^{\prime} \cdot \frac{\partial}{\partial \mathbf{v}}\left(\frac{\mathbf{k} \cdot \partial F_{e} / \partial \mathbf{v}}{\sigma \omega_{\mathbf{k}}^{L}}\right)+\mathbf{k} \cdot \mathbf{v}\left(\frac{2}{v_{T e}^{2}}\right)^{2} F_{e}\right]=0, \\
& \bar{\chi}_{i}^{(3)}\left(\mathbf{k}^{\prime}, 0\left|-\mathbf{k}^{\prime}, 0\right| \mathbf{k}, \sigma \omega_{\mathbf{k}}^{L}\right)=\frac{1}{2} \frac{e^{2}}{m_{e} m_{i}} \frac{1}{k^{2} k^{\prime 2}} \int d \mathbf{v} \mathbf{k}^{\prime} \cdot \frac{\partial}{\partial \mathbf{v}}\left[\mathbf{k}^{\prime} \cdot \frac{\partial}{\partial \mathbf{v}}\left(\frac{\mathbf{k} \cdot \partial F_{i} / \partial \mathbf{v}}{\sigma \omega_{\mathbf{k}}^{L}}\right)+\mathbf{k} \cdot \mathbf{v}\left(\frac{2}{v_{T i}^{2}}\right)^{2} F_{i}\right]=0,
\end{aligned}
$$

where we have taken the partial integrations. For $\omega=\sigma \omega_{\mathbf{k}}^{S}$, since $\omega_{\mathbf{k}}^{S} \ll k v_{T e}$, the electron susceptibility can be approximated by

$$
\bar{\chi}_{e}^{(3)}\left(\mathbf{k}^{\prime}, 0\left|-\mathbf{k}^{\prime}, 0\right| \mathbf{k}, \omega_{\mathbf{k}}^{S}\right) \approx \bar{\chi}_{e}^{(3)}\left(\mathbf{k}^{\prime}, 0\left|-\mathbf{k}^{\prime}, 0\right| \mathbf{k}, 0\right)=-\frac{e^{2}}{2 T_{e}^{2}} \frac{\mathbf{k} \cdot \mathbf{k}^{\prime}}{k^{2}} \frac{1}{k^{2} k^{\prime 2} \lambda_{D e}^{2}} .
$$

For the ions, we have the same situation as Eq. (4.37), namely, two slow modes and a fast mode:

$$
\bar{\chi}_{i}^{(3)}\left(\mathbf{k}^{\prime}, 0\left|-\mathbf{k}^{\prime}, 0\right| \mathbf{k}, \sigma \omega_{\mathbf{k}}^{S}\right)=\frac{1}{2} \frac{e^{2}}{m_{i}^{2}} \frac{\omega_{p i}^{2}}{k^{2} k^{\prime 2}} \frac{1}{\left(\omega_{\mathbf{k}}^{S}\right)^{2}} \int d \mathbf{v} \mathbf{k}^{\prime} \cdot \frac{\partial}{\partial \mathbf{v}}\left[\mathbf{k}^{\prime} \cdot \frac{\partial}{\partial \mathbf{v}}\left(\frac{\mathbf{k} \cdot \partial F_{i} / \partial \mathbf{v}}{\sigma \omega_{\mathbf{k}}^{S}}\right)+\mathbf{k} \cdot \mathbf{v}\left(\frac{2}{v_{T i}^{2}}\right)^{2} F_{i}\right]=0
$$

The approximations (4.36)-(4.40) lead to the simplifications of collisional damping terms (4.35):

$$
\begin{aligned}
\left.\frac{\partial I_{\mathbf{k}}^{\sigma L}}{\partial t}\right|_{\text {coll }} & =-\frac{8 \hat{n} e^{4}}{\pi T_{e}^{2}} \sigma \omega_{\mathbf{k}}^{L} \int d \mathbf{k}^{\prime} \frac{\left[\mathbf{k} \cdot\left(\mathbf{k}-\mathbf{k}^{\prime}\right)\right]^{2} \lambda_{D e}^{4}}{k^{2}\left|\mathbf{k}-\mathbf{k}^{\prime}\right|^{2}\left|1+T_{e} / T_{i}+k^{\prime 2} \lambda_{D e}^{2}\right|^{2}} \frac{\operatorname{Im} \epsilon\left(\mathbf{k}-\mathbf{k}^{\prime}, \sigma \omega_{\mathbf{k}}^{L}\right)}{\left|\epsilon\left(\mathbf{k}-\mathbf{k}^{\prime}, \sigma \omega_{\mathbf{k}}^{L}\right)\right|^{2}} I_{\mathbf{k}}^{\sigma L}, \\
\left.\frac{\partial I_{\mathbf{k}}^{\sigma S}}{\partial t}\right|_{\text {coll }} & =-\frac{2 \hat{n} e^{4}}{\pi T_{e}^{2}} \sigma \mu_{\mathbf{k}} \omega_{\mathbf{k}}^{L} \int d \mathbf{k}^{\prime} \frac{1}{k^{2}\left|\mathbf{k}-\mathbf{k}^{\prime}\right|^{2}\left|1+T_{e} / T_{i}+k^{\prime 2} \lambda_{D e}^{2}\right|^{2}}\left[1+\frac{2 T_{e}}{T_{i}} \frac{\mathbf{k} \cdot\left(\mathbf{k}-\mathbf{k}^{\prime}\right)}{k^{2}}\right] \frac{\operatorname{Im} \epsilon\left(\mathbf{k}-\mathbf{k}^{\prime}, \sigma \omega_{\mathbf{k}}^{S}\right)}{\left|\epsilon\left(\mathbf{k}-\mathbf{k}^{\prime}, \sigma \omega_{\mathbf{k}}^{S}\right)\right|^{2}} I_{\mathbf{k}}^{\sigma S} .
\end{aligned}
$$

Making use of the definition (2.39), we may write down the final form of the partial wave kinetic equation corresponding to the collisional damping terms:

$$
\begin{aligned}
\left.\frac{\partial I_{\mathbf{k}}^{\sigma L}}{\partial t}\right|_{\text {coll }}= & \frac{8 \hat{n} e^{4} \omega_{p e}^{2}}{T_{e}^{2}} \sigma \omega_{\mathbf{k}}^{L} \int d \mathbf{k}^{\prime} \frac{\left[\mathbf{k} \cdot\left(\mathbf{k}-\mathbf{k}^{\prime}\right)\right]^{2} \lambda_{D e}^{4}}{k^{2}\left|\mathbf{k}-\mathbf{k}^{\prime}\right|^{4}\left|\epsilon\left(\mathbf{k}-\mathbf{k}^{\prime}, \sigma \omega_{\mathbf{k}}^{L}\right)\right|^{2}\left|1+T_{e} / T_{i}+k^{\prime 2} \lambda_{D e}^{2}\right|^{2}} \\
& \times \int d \mathbf{v}\left(\mathbf{k}-\mathbf{k}^{\prime}\right) \cdot \frac{\partial F_{e}(\mathbf{v})}{\partial \mathbf{v}} \delta\left[\sigma \omega_{\mathbf{k}}^{L}-\left(\mathbf{k}-\mathbf{k}^{\prime}\right) \cdot \mathbf{v}\right] I_{\mathbf{k}}^{\sigma L}, \\
\left.\frac{\partial I_{\mathbf{k}}^{\sigma S}}{\partial t}\right|_{\text {coll }}= & \sum_{a} \frac{2 \hat{n} e^{4} \omega_{p a}^{2}}{T_{e}^{2}} \sigma \mu_{\mathbf{k}} \omega_{\mathbf{k}}^{L} \int d \mathbf{k}^{\prime} \frac{1}{k^{2}\left|\mathbf{k}-\mathbf{k}^{\prime}\right|^{4}\left|\epsilon\left(\mathbf{k}-\mathbf{k}^{\prime}, \sigma \omega_{\mathbf{k}}^{S}\right)\right|^{2}\left|1+T_{e} / T_{i}+k^{\prime 2} \lambda_{D e}^{2}\right|^{2}} \\
& \times\left[1+\frac{2 T_{e}}{T_{i}} \frac{\mathbf{k} \cdot\left(\mathbf{k}-\mathbf{k}^{\prime}\right)}{k^{2}}\right]^{2} \int d \mathbf{v}\left(\mathbf{k}-\mathbf{k}^{\prime}\right) \cdot \frac{\partial F_{a}(\mathbf{v})}{\partial \mathbf{v}} \delta\left[\sigma \omega_{\mathbf{k}}^{S}-\left(\mathbf{k}-\mathbf{k}^{\prime}\right) \cdot \mathbf{v}\right] I_{\mathbf{k}}^{\sigma S},
\end{aligned}
$$

where we have a trivial change of integral variable, $\mathbf{k}^{\prime} \leftrightarrow \mathbf{k}-\mathbf{k}^{\prime}$.

Collecting all the results, we may finally write the generalized wave kinetic equation that includes contributions from noneigenmode fluctuations, and which directly extends the customary weak turbulence wave kinetic equation (3.22)-(3.27),

$$
\begin{aligned}
\frac{\partial I_{\mathbf{k}}^{\sigma L}}{\partial t}= & S_{\mathbf{k}}^{\sigma L}+P_{\mathbf{k}}^{\sigma L}+2\left(\gamma_{\mathbf{k}}^{\sigma L}+\gamma_{\mathbf{k}}^{\sigma L(\mathrm{coll})}\right) I_{\mathbf{k}}^{\sigma L}+2 \sum_{\sigma^{\prime}, \sigma^{\prime \prime}} \int d \mathbf{k}^{\prime} v_{\mathbf{k}, \mathbf{k}^{\prime}}^{L}\left(\sigma \omega \omega_{\mathbf{k}}^{L} I_{\mathbf{k}^{\prime}}^{\sigma^{\prime} L} I_{\mathbf{k}-\mathbf{k}^{\prime}}^{\sigma^{\prime \prime} S}-\sigma^{\prime} \omega_{\mathbf{k}^{\prime}}^{L} I_{\mathbf{k}-\mathbf{k}^{\prime}}^{\sigma^{\prime \prime} S} I_{\mathbf{k}}^{\sigma L}-\sigma^{\prime \prime} \omega_{\mathbf{k}-\mathbf{k}^{\prime}}^{L} I_{\mathbf{k}^{\prime}}^{\sigma^{\prime} L} I_{\mathbf{k}}^{\sigma L}\right) \\
& -\sum_{\sigma^{\prime}} \int d \mathbf{k}^{\prime}\left[u_{\mathbf{k}, \mathbf{k}^{\prime}}^{L}\left(\sigma^{\prime} \omega_{\mathbf{k}^{\prime}}^{L} I_{\mathbf{k}}^{\sigma L}-\sigma \omega_{\mathbf{k}}^{L} I_{\mathbf{k}^{\prime}}^{\sigma^{\prime} L}\right)-w_{\mathbf{k}, \mathbf{k}^{\prime}}^{L} I_{\mathbf{k}^{\prime}}^{\sigma^{\prime} L} I_{\mathbf{k}}^{\sigma L}\right],
\end{aligned}
$$




$$
\begin{aligned}
\frac{\partial I_{\mathbf{k}}^{\sigma S}}{\partial t}= & S_{\mathbf{k}}^{\sigma S}+P_{\mathbf{k}}^{\sigma S}+2\left(\gamma_{\mathbf{k}}^{\sigma S}+\gamma_{\mathbf{k}}^{\sigma S(\mathrm{coll})}\right) I_{\mathbf{k}}^{\sigma S}+\sum_{\sigma^{\prime}, \sigma^{\prime \prime}= \pm 1} \int d \mathbf{k}^{\prime} v_{\mathbf{k}, \mathbf{k}^{\prime}}^{S}\left(\sigma \omega_{\mathbf{k}}^{L} I_{\mathbf{k}^{\prime}}^{\sigma^{\prime} L} I_{\mathbf{k}-\mathbf{k}^{\prime}}^{\sigma^{\prime \prime} L}-\sigma^{\prime} \omega_{\mathbf{k}^{\prime}}^{L} I_{\mathbf{k}-\mathbf{k}^{\prime}}^{\sigma^{\prime \prime} L} I_{\mathbf{k}}^{\sigma S}-\sigma^{\prime \prime} \omega_{\mathbf{k}-\mathbf{k}^{\prime}}^{L} \sigma_{\mathbf{k}^{\prime}}^{\sigma^{\prime} L} I_{\mathbf{k}}^{\sigma S}\right) \\
& -\sum_{\sigma^{\prime}} \int d \mathbf{k}^{\prime}\left[u_{\mathbf{k}, \mathbf{k}^{\prime}}^{S}\left(\sigma^{\prime} \omega_{\mathbf{k}^{\prime}}^{L} \mathbf{k}_{\mathbf{k}}^{\sigma S}-\sigma \omega_{\mathbf{k}}^{L} I_{\mathbf{k}^{\prime}}^{\sigma^{\prime} S}\right)-w_{\mathbf{k}, \mathbf{k}^{\prime}}^{S} \mathbf{k}_{\mathbf{k}^{\prime}}^{\sigma^{\prime} S} I_{\mathbf{k}}^{\sigma S}\right]
\end{aligned}
$$

where the spontaneous emission terms $S_{\mathbf{k}}^{\sigma L}$ and $S_{\mathbf{k}}^{\sigma S}$ are already defined in Eq. (3.23), but a new term that represents the electrostatic bremsstrahlung appears in Eq. (4.42), namely,

$$
\begin{aligned}
P_{\mathbf{k}}^{\sigma L}= & \frac{3 e^{2} T_{e}}{16 \pi^{3} m_{e}}\left(1-\frac{T_{e}^{2}}{T_{i}^{2}}\right)^{2} \frac{1}{k^{2} \lambda_{D e}^{2}} \int d \mathbf{k}^{\prime}\left(1+\frac{T_{e}}{T_{i}}+k^{\prime 2} \lambda_{D e}^{2}\right)^{-2}\left[1+\frac{T_{e}}{T_{i}}+\left(\mathbf{k}-\mathbf{k}^{\prime}\right)^{2} \lambda_{D e}^{2}\right]^{-2} \\
& \times \int d \mathbf{v} \int d \mathbf{v}^{\prime} \delta\left[\sigma \omega_{\mathbf{k}}^{L}-\mathbf{k} \cdot \mathbf{v}+\mathbf{k}^{\prime} \cdot\left(\mathbf{v}-\mathbf{v}^{\prime}\right)\right] \sum_{a} F_{a}(\mathbf{v}) \sum_{b} F_{b}\left(\mathbf{v}^{\prime}\right), \\
P_{\mathbf{k}}^{\sigma S}= & \mu_{\mathbf{k}} \frac{3 e^{2} T_{e}}{16 \pi^{3} m_{e}}\left(1-\frac{T_{e}^{2}}{T_{i}^{2}}\right)^{2} \frac{1}{k^{2} \lambda_{D e}^{2}} \int d \mathbf{k}^{\prime}\left(1+\frac{T_{e}}{T_{i}}+{k^{\prime}}^{2} \lambda_{D e}^{2}\right)^{-2}\left[1+\frac{T_{e}}{T_{i}}+\left(\mathbf{k}-\mathbf{k}^{\prime}\right)^{2} \lambda_{D e}^{2}\right]^{-2} \\
& \times \int d \mathbf{v} \int d \mathbf{v}^{\prime} \delta\left[\sigma \omega_{\mathbf{k}}^{S}-\mathbf{k} \cdot \mathbf{v}+\mathbf{k}^{\prime} \cdot\left(\mathbf{v}-\mathbf{v}^{\prime}\right)\right] \sum_{a} F_{a}(\mathbf{v}) \sum_{b} F_{b}\left(\mathbf{v}^{\prime}\right) .
\end{aligned}
$$

The collisionless (Landau) damping rates $\gamma_{\mathbf{k}}^{\sigma L}$ and $\gamma_{\mathbf{k}}^{\sigma S}$ are also already defined in Eq. (3.24), but Eq. (4.42) now contains new terms associated with collisional damping rates:

$$
\begin{aligned}
\gamma_{\mathbf{k}}^{\sigma L(\text { coll })}= & \sigma \omega_{\mathbf{k}}^{L} \frac{4 \hat{n} e^{4} \omega_{p e}^{2}}{T_{e}^{2}} \int d \mathbf{k}^{\prime} \frac{\left(\mathbf{k} \cdot \mathbf{k}^{\prime}\right)^{2} \lambda_{D e}^{4}}{k^{2} k^{\prime 4}\left|\epsilon\left(\mathbf{k}^{\prime}, \sigma \omega_{\mathbf{k}}^{L}\right)\right|^{2}}\left[1+\frac{T_{e}}{T_{i}}+\left(\mathbf{k}-\mathbf{k}^{\prime}\right)^{2} \lambda_{D e}^{2}\right]^{-2} \int d \mathbf{v} \mathbf{k}^{\prime} \cdot \frac{\partial F_{e}(\mathbf{v})}{\partial \mathbf{v}} \delta\left(\sigma \omega_{\mathbf{k}}^{L}-\mathbf{k}^{\prime} \cdot \mathbf{v}\right), \\
\gamma_{\mathbf{k}}^{\sigma S(\text { coll })}= & \sigma \mu_{\mathbf{k}} \omega_{\mathbf{k}}^{L} \frac{\hat{n} e^{4} \omega_{p e}^{2}}{T_{e}^{2}} \int d \mathbf{k}^{\prime} \frac{1}{k^{2} k^{\prime 4}\left|\epsilon\left(\mathbf{k}^{\prime}, \sigma \omega_{\mathbf{k}}^{S}\right)\right|^{2}}\left[1+\frac{T_{e}}{T_{i}}+\left(\mathbf{k}-\mathbf{k}^{\prime}\right)^{2} \lambda_{D e}^{2}\right]^{-2} \\
& \times\left(1+\frac{2 T_{e}}{T_{i}} \frac{\mathbf{k} \cdot \mathbf{k}^{\prime}}{k^{2}}\right) \int d \mathbf{v} \mathbf{k}^{\prime} \cdot \frac{\partial}{\partial \mathbf{v}}\left[F_{e}(\mathbf{v})+\frac{m_{e}}{m_{i}} F_{i}(\mathbf{v})\right] \delta\left(\sigma \omega_{\mathbf{k}}^{S}-\mathbf{k}^{\prime} \cdot \mathbf{v}\right) .
\end{aligned}
$$

The decay coefficients $v_{\mathbf{k}, \mathbf{k}^{\prime}}^{L}$ and $v_{\mathbf{k}, \mathbf{k}^{\prime}}^{S}$ are identical to those defined in Eq. (3.25). However, the coefficients describing the spontaneous scattering terms, $u_{\mathbf{k}, \mathbf{k}^{\prime}}^{L}$ and $u_{\mathbf{k}, \mathbf{k}^{\prime}}^{S}$, are generalized to include the effects of noneigenmode electrostatic fluctuations:

$$
\begin{aligned}
u_{\mathbf{k}, \mathbf{k}^{\prime}}^{L}= & \sigma \omega_{\mathbf{k}}^{L} \frac{\hat{n} e^{4}}{m_{e}^{2} \omega_{p e}^{4}} \frac{\left(\mathbf{k} \cdot \mathbf{k}^{\prime}\right)^{2}}{k^{2} k^{\prime 2}}\left[1+\frac{1}{\left|1+T_{e} / T_{i}+\left(\mathbf{k}-\mathbf{k}^{\prime}\right)^{2} \lambda_{D e}^{2}\right|^{2}}\right] \int d \mathbf{v} \delta\left[\sigma \omega_{\mathbf{k}}^{L}-\sigma^{\prime} \omega_{\mathbf{k}^{\prime}}^{L}-\left(\mathbf{k}-\mathbf{k}^{\prime}\right) \cdot \mathbf{v}\right]\left[F_{e}(\mathbf{v})+F_{i}(\mathbf{v})\right], \\
u_{\mathbf{k}, \mathbf{k}^{\prime}}^{S}= & \mu_{\mathbf{k}} \mu_{\mathbf{k}^{\prime}} \sigma \omega_{\mathbf{k}}^{L} \frac{\hat{n} e^{4}}{m_{e}^{2} \omega_{p e}^{4}} \frac{1}{k^{2} k^{\prime 2} \lambda_{D e}^{4}}\left[\frac{\left(\mathbf{k} \cdot \mathbf{k}^{\prime}\right)^{2}}{k^{2} k^{\prime 2}} W_{\mathbf{k}, \mathbf{k}^{\prime}}+\frac{1}{\left|1+T_{e} / T_{i}+\left(\mathbf{k}-\mathbf{k}^{\prime}\right)^{2} \lambda_{D e}^{2}\right|^{2}}\left(1-\frac{T_{e} \mathbf{k} \cdot \mathbf{k}^{\prime}}{T_{i}} \frac{2}{k^{\prime 2}}\right)^{2}\right] \\
& \times \int d \mathbf{v} \delta\left[\sigma \omega_{\mathbf{k}}^{S}-\sigma^{\prime} \omega_{\mathbf{k}^{\prime}}^{S}-\left(\mathbf{k}-\mathbf{k}^{\prime}\right) \cdot \mathbf{v}\right]\left[F_{e}(\mathbf{v})+F_{i}(\mathbf{v})\right] .
\end{aligned}
$$

Finally, the coefficients associated with the induced scattering terms, $w_{\mathbf{k}, \mathbf{k}^{\prime}}^{L}$ and $w_{\mathbf{k}, \mathbf{k}^{\prime}}^{S}$, remain the same as defined in Eq. (3.27).

\section{SUMMARY AND DISCUSSION}

The present paper formulated the weak turbulence theory that includes effects of binary collisions as well as collective processes. For the sake of simplicity, unmagnetized plasmas interacting through electrostatic force were considered. In spite of this simplification, the new formulation presented in this paper extends the customary formalism found in the literature. The new aspect of the theory pertains to the issue of treating the spontaneously-emitted electrostatic fluctuations in a complete fashion. The spontaneous emission is a discrete-particle effect, and thermal plasmas emit such fluctuations in all frequencies and wave vectors $(\omega, \mathbf{k})$. However, in the customary literature, these fluctuations are approximately treated by considering only those frequency-wave number regimes corresponding to the eigenmodes, $\omega=\omega_{\mathbf{k}}$, while ignoring contributions from noneigenmodes, $\omega \neq \omega_{\mathbf{k}}$. In the present paper, we include the noneigenmode contribution to the electrostatic fluctuations and rigorously show that a systematic weak turbulence theory that encompasses collisional processes emerges.

In the literature, collisional effects are sometimes included in the particle kinetic equation, but not in conjunction with and within the context of the weak turbulence theory, developed from first principles. It is customary simply to add a collisional term to the right-hand side of a linearized particle kinetic equation, approach which is particularly common in the literature that deals with the collisional transport processes in fusion research. On the other hand, in the literature related to the analysis of the time 
evolution of wave modes, a collisional damping term is sometimes added to the right-hand side of the wave kinetic equation, with the damping rate directly related to the effective particle collision frequency which is obtained from the particle kinetic equation. However, these procedures found in the literature are not rigorous. The present paper lays out a proper procedure in which the collision integral for the particles and a rigorous wave collisional damping rate are derived on the basis of the Klimontovich system of equations. The result comprises of the particle kinetic equation that contains both the collective effects, spontaneous thermal effects as well as collision integral [Eq. (4.18)], and the generalized wave kinetic equation that contains the collective linear and nonlinear wave-particle interaction terms, nonlinear wave-wave interaction terms, collisional damping terms, as well as terms that depict the bremsstrahlung emission of plasma eigenmodes [Eq. (4.42)].

\section{ACKNOWLEDGMENTS}

P.H.Y. acknowledges NSF grant AGS1550566 to the University of Maryland and the support by the BK21 plus program through the National Research Foundation (NRF) funded by the Ministry of Education of Korea, to Kyung Hee University, Korea. He also acknowledges the Science Award Grant from the GFT Foundation to the University of Maryland. Part of this work was carried out while P.H.Y. was visiting the Ruhr University Bochum (RUB), Germany, which was made possible by support from the Ruhr University Research School PLUS, funded by Germany's Excellence Initiative (DFG GSC 98/3), and the Visiting International Professor Fellowship administered by RUB. This work has been partially supported by the Brazilian agencies CNPq and FAPERGS. E.P.K. was supported via STFC consolidated grant. The work by R.S. was supported by the Deutsche Forschungsgemeinschaft through grant Schl 201/32-1.

[1] B. B. Kadomtsev, Plasma Turbulence (Academic Press, New York, 1965).

[2] A. A. Vedenov, A. V. Gordeev, and L. I. Rudakov, Plasma Phys. 9, 719 (1967).

[3] R. Z. Sagdeev and A. A. Galeev, Nonlinear Plasma Theory (Benjamin, New York, 1969).

[4] V. N. Tsytovich, Nonlinear Effects in a Plasma (Plenum, New York, 1970).

[5] R. C. Davidson, Methods in Nonlinear Plasma Theory (Academic Press, New York, 1972).

[6] A. I. Akhiezer, I. A. Akhiezer, R. V. Polovin, A. G. Sitenko, and K. N. Stepanov, Plasma Electrodynamics, vol. 2, Non-Linear Theory and Fluctuations (Pergamon, New York, 1975).

[7] V. N. Tsytovich, An Introduction to the Theory of Plasma Turbulence (Pergamon, New York, 1977).

[8] D. B. Melrose, Plasma Astrophysics (Gordon and Breach, New York, 1980).

[9] A. G. Sitenko, Fluctuations and Nonlinear Wave Interactions in Plasmas (Pergamon, New York, 1982).

[10] V. N. Tsytovich, Lectures on Nonlinear Plasma Kinetics (Springer, New York, 1995).

[11] R. J. Grognard, Solar Phys. 81, 173 (1982).

[12] A. Hanssen, J. Geophys. Res. 96, 1867 (1991).

[13] S. D. Edney and P. A. Robinson, Phys. Plasmas 8, 428 (2001).

[14] E. P. Kontar, Solar Phys. 202, 131 (2001).

[15] E. P. Kontar and H. L. Pécseli, Phys. Rev. E 65, 066408 (2002).

[16] P. H. Yoon, Phys. Plasmas 7, 4858 (2000).

[17] P. H. Yoon, Phys. Plasmas 12, 042306 (2005).

[18] P. H. Yoon, Phys. Plasmas 13, 022302 (2006).

[19] P. H. Yoon, S. Yi, and C.-M. Ryu, Phys. Plasmas 12, 052305 (2005).

[20] P. H. Yoon, L. F. Ziebell, R. Gaelzer, and J. Pavan, Phys. Plasmas 19, 102303 (2012).

[21] L. F. Ziebell, R. Gaelzer, and P. H. Yoon, Phys. Plasmas 8, 3982 (2001).

[22] P. H. Yoon, T. Rhee, and C.-M. Ryu, Phys. Rev. Lett. 95, 215003 (2005).
[23] L. F. Ziebell, R. Gaelzer, J. Pavan, and P. H. Yoon, Plasma Phys. Contr. Fusion 50, 085011 (2008).

[24] R. Gaelzer, L. F. Ziebell, A. Figueroa-Viñas, P. H. Yoon, and C.-M. Ryu, Astrophys. J. 677, 676 (2008).

[25] R. Gaelzer, P. H. Yoon, L. F. Ziebell, and A. F.-Viñas, Adv. Geosci. 14, 111 (2007).

[26] P. H. Yoon, J. Hong, S. Kim, J. Lee, J. Lee, J. Park, K. S. Park, and J. J. Seough, Astrophys J. 754, 181803 (2012).

[27] P. H. Yoon, Phys. Plasmas 19, 052301 (2012).

[28] L. F. Ziebell, P. H. Yoon, R. Gaelzer, and J. Pavan, Plasma Phys. Contr. Fusion 54, 055012 (2012).

[29] P. H. Yoon, L. F. Ziebell, R. Gaelzer, R. P. Lin, and L. Wang, Space Sci. Rev. 173, 459 (2012).

[30] L. F. Ziebell, P. H. Yoon, F. J. R. S. Jr., R. Gaelzer, and J. Pavan, Phys. Plasmas 21, 010701 (2014).

[31] L. F. Ziebell, P. H. Yoon, R. Gaelzer, and J. Pavan, Astrophys. J. Lett. 795, L32 (2014).

[32] L. F. Ziebell, P. H. Yoon, R. Gaelzer, and J. Pavan, Phys. Plasmas 21, 012306 (2014).

[33] J. C. Brown, Solar Phys. 18, 489 (1971).

[34] S. I. Syrovatskii and O. P. Shmeleva, Soviet Ast. 16, 273 (1972).

[35] G. D. Holman, M. J. Aschwanden, H. Aurass, M. Battaglia, P. C. Grigis, E. P. Kontar, W. Liu, P. Saint-Hilaire, and V. V. Zharkova, Space Sci. Rev. 159, 107 (2011).

[36] A. G. Emslie and D. F. Smith, Astrophys. J. 279, 882 (1984).

[37] E. P. Kontar, H. Ratcliffe, and N. H. Bian, Astron. Astrophys. 539, A43 (2012).

[38] I. G. Hannah, E. P. Kontar, and O. K. Sirenko, Astrophys. J. Lett. 707, L45 (2009).

[39] I. G. Hannah, E. P. Kontar, and H. A. S. Reid, Astron. Astrophys. 550, A51 (2013).

[40] S. D. Baalrud, J. D. Callen, and C. C. Hegna, Phys. Plasmas 15, 092111 (2008).

[41] H. Ratcliffe and E. P. Kontar, Astron. Astrophys. 562, A57 (2014).

[42] W. P. Wood and B. W. Ninham, Aust. J. Phys. 22, 453 (1969). 
[43] I. Fidone, R. L. Meyer, and G. Granata, Phys. Fluids 26, 3292 (1983).

[44] I. Fidone, G. Giruzzi, G. Granata, and R. L. Meyer, Phys. Fluids 27, 2468 (1984).

[45] G. Giruzzi, V. Krivenski, I. Fidone, and L. F. Ziebell, Plasma Phys. Contr. Fusion 27, 1151 (1985).

[46] A. G. Peeters and E. Westerhof, Phys. Plasmas 3, 1628 (1996).

[47] I. P. Shkarofsky and M. M. Shoucri, Nucl. Fusion 37, 539 (1997).

[48] P. H. Diamond, S.-I. Itoh, and K. Itoh, Modern Plasma Physics, Vol. 1: Physical Kinetics of Turbulent Plasmas (Cambridge University Press, New York, 2010).

[49] W. D. McComb, The Physics of Fluid Turbulence (Oxford University Press, New York, 1990).

[50] P. H. Yoon, C.-M. Ryu, and T. Rhee, Phys. Plasmas 10, 3881 (2003).
[51] P. H. Yoon and R. Gaelzer, Phys. Plasmas 9, 4166 (2002).

[52] P. H. Yoon, R. Gaelzer, T. Umeda, Y. Omura, and H. Matsumoto, Phys. Plasmas 19, 364 (2003).

[53] A. K. Gailitis and V. N. Tsytovich, Soviet Astron. AJ 8, 359 (1964).

[54] S. A. Colgate, Astrophys. J. 150, 163 (1967).

[55] D. B. Melrose, Astrophys. Space Sci. 10, 197 (1971).

[56] R. A. Windsor and P. J. Kellogg, Astrophys. J. 190, 167 (1974).

[57] A. V. Akopyan and V. N. Tsytovich, Astrofizika 13, 717 (1977).

[58] R. Schlickeiser, Astron. Astrophys. 410, 397 (2003).

[59] J. Dawson and C. Oberman, Phys. Fluids 5, 517 (1962).

[60] T. H. Dupree, Phys. Fluids 6, 1714 (1963).

[61] T. Birmingham, J. Dawson, and C. Oberman, Phys. Fluids 8, 297 (1965).

[62] D. A. Tidman and T. H. Dupree, Phys. Fluids 8, 1860 (1965). 